\title{
Higgs decay into massive $b$-quarks at NNLO QCD in the nested soft-collinear subtraction scheme
}

\author{
Arnd Behring $^{a}$ and Wojciech Bizoń ${ }^{a, b}$ \\ ${ }^{a}$ Institut für Theoretische Teilchenphysik (TTP), KIT, \\ Wolfgang-Gaede-Straße 1, 76131 Karlsruhe, Germany \\ ${ }^{b}$ Institut für Kernphysik (IKP), KIT, \\ Hermann-von-Helmholtz-Platz 1, 76344 Eggenstein-Leopoldshafen, Germany \\ E-mail: arnd.behring@kit.edu, wojciech.bizon@kit.edu
}

ABSTRACT: We present a fully differential description of a decay of a scalar Higgs boson into massive $b$-quarks valid at next-to-next-to-leading order (NNLO) in perturbative quantum chromodynamics (QCD). We work within the nested soft-collinear subtraction scheme extended to accommodate massive partons. We include the loop-induced contribution involving a Higgs coupling to a top quark. We test our calculation against results existing in the literature, comparing the predictions for the total decay width and jet rates.

KeYwords: Heavy Quark Physics, Higgs Physics, Perturbative QCD

ARXIV EPRINT: 1911.11524 


\section{Contents}

1 Introduction 1

2 General considerations 2

2.1 Notation 2

2.2 Outline of the subtraction scheme 3

2.3 IR poles of virtual amplitudes 5

2.4 Phase-space parametrisation $\quad 6$

2.5 Pole vs. $\overline{\mathrm{MS}}$ Yukawa coupling 9

$3 \quad H \rightarrow b \bar{b}$ decay at NLO $\quad 10$

$\begin{array}{lll}3.1 & \text { Real contribution } & 10\end{array}$

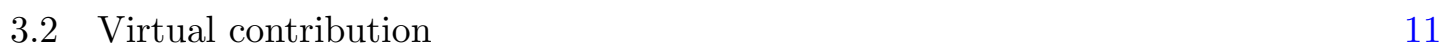

$\begin{array}{lll}3.3 & \text { Pole cancellation } & 12\end{array}$

$4 \quad H \rightarrow b \bar{b}$ decay at NNLO

$\begin{array}{lll}4.1 & \text { Double-real contribution } & 13\end{array}$

$\begin{array}{lll}4.2 & \text { Real-virtual contribution } & 16\end{array}$

$\begin{array}{ll}4.3 \text { Double-virtual contribution } & 17\end{array}$

$\begin{array}{lll}4.4 & \text { Pole cancellation } & 18\end{array}$

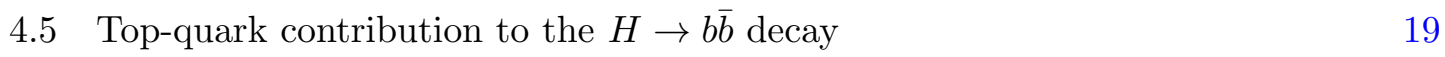

5 Results $\quad 20$

5.1 Overview of the calculation 21

5.2 Total width of the $H \rightarrow b \bar{b}$ decay at NNLO $\quad 21$

5.3 Jet rates for $H \rightarrow b \bar{b}$ at NNLO 23

6 Conclusions 25

$\begin{array}{lr}\text { A Renormalisation } & 26\end{array}$

B Useful formulae $\quad 28$

B.1 Anomalous dimensions for IR factorisation 28

B.2 Coefficients for on-shell to $\overline{\mathrm{MS}}$-scheme conversion 29

C Factorisation formulae $\quad \mathbf{3 0}$

$\begin{array}{ll}\text { C.1 Single-collinear factorisation } 30 & 30\end{array}$

$\begin{array}{lll}\text { C.2 Single-soft factorisation (tree-level) } & 31\end{array}$

C.3 Single-soft factorisation (one-loop) 31

C.4 Double-soft factorisation 32 
D.1 Single-collinear subtraction terms 33

$\begin{array}{lll}\text { D.2 Single-soft subtraction terms (tree-level) } & 35\end{array}$

D.3 Single-soft subtraction terms (one-loop) 38

D.4 Double-soft subtraction terms 39

\section{Introduction}

After the Higgs boson discovery by the ATLAS and CMS collaborations in 2012, the study of Higgs boson properties has become one of the major research avenues in particle physics. Since the mass of the Higgs boson has already been precisely measured [1], all couplings between the Higgs boson and other Standard Model (SM) particles can be accurately predicted. Nevertheless, these couplings can be modified by New Physics that lies beyond the SM. Hence, actual measurements of those couplings can provide important constraints on many extensions of the SM.

The Higgs boson decay into a pair of $b$-quarks is the most common decay channel of the Higgs boson and it is essential to many New Physics searches. Indeed, it plays a particularly important role when considering rare Higgs boson production modes, which benefit from the large $H \rightarrow b \bar{b}$ branching fraction. Although such measurements are often very challenging, due to overwhelming QCD backgrounds, the $H \rightarrow b \bar{b}$ decay has already been observed by both ATLAS and CMS [2,3]. The upcoming years of data taking at the Large Hadron Collider (LHC) will allow for further exploration and use of this Higgs boson decay channel.

In order to fully utilise the data collected at the LHC, a good theoretical understanding of the $H \rightarrow b \bar{b}$ process is required. The next-to-leading order (NLO) QCD corrections have been available for a long time [4-9]. Currently, corrections to the total decay width are known up to $\mathcal{O}\left(\alpha_{s}^{4}\right)$ in the limit of massless $b$-quarks [10]. Mass effects at $\mathcal{O}\left(\alpha_{s}^{2}\right)$ have been estimated using a large momentum expansion [11]. Furthermore, the impact of a separate class of corrections from diagrams arising at $\mathcal{O}\left(\alpha_{s}^{2}\right)$ which involve the top-quark Yukawa coupling has been calculated in the limit of a large top-quark mass $[12,13]$ as well as for general values of the masses [14]. Recently, a set of two-loop master integrals required for mixed QCD-electroweak corrections has been computed [15]. In the limit of massless $b$-quarks a number of fully differential next-to-next-to-leading order (NNLO) calculations of the $H \rightarrow b \bar{b}$ decay have been presented [16-19] with first $\mathrm{N}^{3} \mathrm{LO}$ QCD results appearing recently [20]. The $b$-quark mass effects for differential observables have been studied at NNLO QCD in ref. [21].

In this paper, we present an independent calculation of the $b$-quark mass effects in the $H \rightarrow b \bar{b}$ decay at NNLO QCD. Although we believe such a calculation is interesting in its own right and serves as a useful check of the results presented in ref. [21], it is also an essential step towards studying mass effects in associated Higgs production with a vector boson, $p p \rightarrow H V \rightarrow b \bar{b} V$. NNLO QCD corrections to this process have already been studied in the limit of massless $b$-quarks $[18,19,22]$, and large effects, related to radiative 
corrections, have been reported. The impact of the $b$-quark mass may be sizeable in certain regions of the phase space. Higher-order effects in that process have also been investigated using parton showers [23-25]. Another contribution to the Higgs decay width at NNLO is mediated by top-quark loops. In the context of differential distributions, it has been discussed in ref. [18] and subsequently investigated in ref. [14]. A consistent treatment of these contributions requires keeping the $b$-quark mass finite [18].

We work within the nested soft-collinear subtraction scheme [18, 26-28], which is an extension of the original sector-improved residue subtraction scheme [29-32]. To incorporate the $b$-quark masses into the calculation, we rely on the treatment of massive particles outlined in ref. [31].

The paper is organised as follows. In section 2 we introduce the notation and discuss the main steps of the subtraction scheme. We also describe the infrared (IR) poles appearing in virtual amplitudes and touch upon the relation between the pole and $\overline{\mathrm{MS}}$ Yukawa couplings. In section 3 and section 4 we review the NLO QCD calculation of the $H \rightarrow b \bar{b}$ decay and present our calculation of the NNLO corrections, including the treatment of the top-quark induced corrections. Finally, in section 5, we thoroughly test our predictions against results available in the literature. We summarise our findings in section 6 . The appendices contain a number of expressions used throughout our calculation.

\section{General considerations}

We are interested in decays of a scalar Higgs boson into a pair of $b$-quarks. Our goal is to treat $b$-quarks as massive particles throughout the calculation and achieve NNLO accuracy in perturbative QCD while working within the nested soft-collinear subtraction scheme $[18,26-28]$.

\subsection{Notation}

We start with a short introduction that will set the stage for our calculation. We consider the Higgs boson decaying into a pair of $b$-quarks

$$
H\left(q_{1}\right) \longrightarrow b\left(q_{2}\right)+\bar{b}\left(q_{3}\right)
$$

with $q_{1}^{2}=M_{H}^{2}$ and $q_{2}^{2}=q_{3}^{2}=m_{b}^{2}$. The leading order (LO) partial decay width of the Higgs boson into a pair of $b$-quarks is

$$
\Gamma_{\mathrm{LO}}=\frac{1}{2 M_{H}} \int \mathrm{d} \Phi_{b \bar{b}}\left(q_{1}\right)\left|\mathcal{M}_{b \bar{b}}^{(0)}\right|^{2},
$$

where $\mathrm{d} \Phi_{b \bar{b}}\left(q_{1}\right)$ is the two-particle phase-space volume element for the production of two $b$-quarks with total momentum $q_{1}$, and $\left|\mathcal{M}_{b \bar{b}}^{(0)}\right|^{2}$ is the squared tree-level matrix element. For brevity, we list only final-state particles in the sub- and superscripts, since the initial state is always an on-shell Higgs boson at rest. Upon integration we obtain

$$
\Gamma_{\mathrm{LO}}=\frac{N_{c}}{16 \pi} y_{b}^{2} M_{H} \beta^{3},
$$


where $N_{c}=3$ is the number of colours, $\beta=\sqrt{1-4 m_{b}^{2} / M_{H}^{2}}$ and $y_{b}$ stands for the $b$-quark Yukawa coupling, $y_{b}=m_{b}\left(2 \sqrt{2} G_{F}\right)^{1 / 2}$.

The Higgs boson decay width into $b$-quarks receives radiative corrections that can be systematically calculated in perturbative QCD. We use the following notation for the perturbative expansion of the width

$$
\Gamma^{b \bar{b}}=\Gamma_{\mathrm{LO}}^{b \bar{b}}\left[1+\left(\frac{\alpha_{s}}{\pi}\right) \gamma_{1}^{b \bar{b}}+\left(\frac{\alpha_{s}}{\pi}\right)^{2} \gamma_{2}^{b \bar{b}}+\mathcal{O}\left(\alpha_{s}^{3}\right)\right] .
$$

In eq. (2.4), $\alpha_{s}$ is the $\overline{\mathrm{MS}} \mathrm{QCD}$ strong coupling constant defined in a theory with $n_{f}=n_{l}+1$ quark flavours, where $n_{l}$ is the number of massless flavours.

It is useful to define a shorthand notation that denotes an integral over the Lorentzinvariant phase space of particles involved in a particular (sub)process. Similar to the notation in ref. [26], we define

$$
F_{L M}(b \bar{b} X)=\mathrm{d} \Phi_{b \bar{b} X}\left(q_{1}\right)\left|\mathcal{M}_{b \bar{b} X}^{(0)}\right|^{2} \mathcal{F}_{\text {kin }}(b \bar{b} X)
$$

where $b \bar{b} X$ denotes the constituents of the final state of a considered subprocess, $\mathrm{d} \Phi_{b \bar{b} X}\left(q_{1}\right)$ and $\left|\mathcal{M}_{b \bar{b} X}^{(0)}\right|^{2}$ stand for the Lorentz-invariant phase-space measure and the squared amplitude of the $H \rightarrow b \bar{b} X$ process, respectively. The momentum $q_{1}$ refers to the initial state Higgs boson, while $\mathcal{F}_{\text {kin }}$ is an infrared-safe observable that depends on the kinematical configuration of the particles involved in the process. We will use the notation

$$
\langle A\rangle=\int \mathrm{d} \Phi A
$$

to denote the integration of some quantity $A$ over the phase space, $\mathrm{d} \Phi$.

\subsection{Outline of the subtraction scheme}

One of the challenges in higher-order QCD calculations is the appearance of infrared singularities when massless particles become soft or collinear. Dimensional regularisation [33-37] can be used to regulate these singularities which show up as poles in the dimensional regularisation parameter $\epsilon=(4-d) / 2$ in both real and virtual amplitudes. For an infrared-safe observable these poles cancel between real and virtual corrections and collinear factorisation contributions once the loop and phase-space integrals over the singular regions are performed [38, 39]. The observables depend on the momenta of the real emission partons so that numerical integration of the phase-space integrals is desirable from the standpoint of flexibility and often also required due to the complexity of the observable, which may involve, e.g., complicated kinematic constraints and jet algorithms.

It follows from the factorisation theorems of QCD that the integrand of the crosssection, i.e., the combination of squared matrix elements and phase-space measure, scales as $E_{i}^{-1+a \epsilon} \mathrm{d} E_{i}$ in soft limits and as $\theta_{i j}^{-1+b \epsilon} \mathrm{d} \theta_{i j}$ in collinear limits of massless partons, where $E_{i}$ is the energy of the soft parton, $\theta_{i j}$ is the angle between the collinear partons and $a, b \in \mathbb{R}$. Thus, $\epsilon$ acts as a regulator for logarithmic divergences in the real-emission phasespace integrals. For the numerical integration it is necessary to explicitly extract and cancel 
the poles in $\epsilon^{-1}$ and to regulate the integrals in such a way that the expansion in $\epsilon$ can be performed at the integrand level.

A number of methods have been developed to accomplish that. Here, we follow the nested soft-collinear subtraction scheme $[18,26-28]$ which is closely related to the sectorimproved residue subtraction scheme [29-32]. As with all subtraction schemes, the general idea is to introduce subtraction terms for each singular limit which regulate the integrand and to add back these subtraction terms integrated over the unresolved phase space. Schematically, for an integral of a function $F$ over the phase space, we write

$$
\langle F\rangle=\langle F-O F\rangle+\langle O F\rangle,
$$

where $O$ is an operator which extracts the asymptotic behaviour of $F$ and the phase space in a singular limit. The term $\langle O F\rangle$, which is integrated over the unresolved phase space in $d$ dimensions, then carries explicit poles in $\epsilon^{-1}$, while the regulated term $\langle F-O F\rangle$ is expanded in $\epsilon$ at the integrand level. We apply eq. (2.7) recursively to regulate all singular limits.

The nested soft-collinear subtraction scheme consists of the following steps.

1. Introduce subtraction terms for the soft limits. At NNLO this involves up to two single-soft limits and the double-soft limit.

2. Introduce a partition of unity for the phase space that isolates the collinear limits, $1=\sum_{\{p\}} w_{\{p\}}$, where the sum runs over the sets of partons that can produce collinear singularities and the functions $w_{\{p\}}$ go to zero whenever two partons which are not in $p$ become collinear, thereby regulating integrand in that limit.

3. In each collinear partition, choose a suitable phase-space parametrisation in terms of angles and energies of the partons that can become unresolved.

4. Use sector decomposition [40-42] to map all singularities to the boundaries of the region of integration so that the singularities can be easily extracted upon using eq. (2.7). In order to generate the limits of the matrix elements, we use the standard QCD factorisation formulae for the soft and collinear limits. All necessary expressions up to NNLO can be found, e.g., in the appendices of ref. [31].

The $H \rightarrow b \bar{b}$ process with massive $b$-quarks is particularly simple in this context since there are no triple- or double-collinear limits that involve $b$-quarks so that step 2 can be avoided. Moreover, by choosing an appropriate phase-space parametrisation in step 3, the sector decomposition of step 4 only yields a single collinear subsector.

The calculation will be subdivided into pieces so that the cancellation of $\epsilon^{-1}$ poles can be shown without making reference to the explicit form of the matrix elements. In particular, we will organise the contributions into sets according to the multiplicity of the resolved final state and the loop order of the matrix elements. By combining this with a suitable phase-space parametrisation which decouples the integrations over the unresolved and resolved parts of phase space, we demonstrate pole cancellation for each phase-space point of the resolved configuration separately. In sections 3 and 4 we explain the application of this scheme to the $H \rightarrow b \bar{b}$ process in greater detail. 


\subsection{IR poles of virtual amplitudes}

The one- and two-loop virtual amplitudes that we encounter in an NNLO calculation feature ultraviolet (UV) divergences that can be removed using a suitable renormalisation procedure. We employ a hybrid scheme in which we renormalise quark and gluon fields, the quark masses and the Yukawa coupling in an on-shell scheme, while we use the $\overline{\mathrm{MS}}$ scheme for the strong coupling. The details of our renormalisation choice are described in section A.

At this point, the renormalised amplitudes are free of UV divergences. Nevertheless, they still contain poles in $\epsilon^{-1}$ which are of IR origin. These poles can be predicted from general considerations [43-51]. They factorise in the form

$$
|\mathcal{M}\rangle=\mathbf{Z}|\mathcal{F}\rangle,
$$

where $|\mathcal{M}\rangle$ is the UV-renormalised amplitude, $\mathbf{Z}$ is an operator in colour space which contains poles in $\epsilon^{-1}$ and $|\mathcal{F}\rangle$ is a finite remainder which does not contain any poles. Expanding all pieces in the strong coupling, we find

$$
\begin{aligned}
|\mathcal{M}\rangle= & \left|\mathcal{M}^{(0)}\right\rangle+\frac{\alpha_{s}}{4 \pi}\left(\mathbf{Z}^{(1)}\left|\mathcal{M}^{(0)}\right\rangle+\left|\mathcal{F}^{(1)}\right\rangle\right) \\
& +\left(\frac{\alpha_{s}}{4 \pi}\right)^{2}\left(\mathbf{Z}^{(2)}\left|\mathcal{M}^{(0)}\right\rangle+\mathbf{Z}^{(1)}\left|\mathcal{F}^{(1)}\right\rangle+\left|\mathcal{F}^{(2)}\right\rangle\right) .
\end{aligned}
$$

Note that this notation leaves all powers of the strong coupling related to real emissions implicit inside the amplitudes. On the one hand, we can use eq. (2.9) as a prediction in order to check the $\epsilon^{-1}$ poles of the UV-renormalised amplitudes. On the other hand, we can also use eq. (2.9) to define the finite remainders, i.e.

$$
\begin{aligned}
\left|\mathcal{F}^{(0)}\right\rangle & =\left|\mathcal{M}^{(0)}\right\rangle \\
\left|\mathcal{F}^{(1)}\right\rangle & =\left|\mathcal{M}^{(1)}\right\rangle-\mathbf{Z}^{(1)}\left|\mathcal{M}^{(0)}\right\rangle \\
\left|\mathcal{F}^{(2)}\right\rangle & =\left|\mathcal{M}^{(2)}\right\rangle-\mathbf{Z}^{(1)}\left|\mathcal{F}^{(1)}\right\rangle-\mathbf{Z}^{(2)}\left|\mathcal{M}^{(0)}\right\rangle,
\end{aligned}
$$

and express all formulae in terms of these. This is useful for showing pole cancellation since it allows us to make the pole terms explicit without specifying the matrix elements that they multiply. The pole terms are multiplied by lower order quantities, as expected. Note that we only include the $\epsilon^{-1}$ poles in the definition of the $\mathbf{Z}$ operator, cf. refs. [46, 51].

In general, $\mathbf{Z}$ is an operator acting on vectors in colour space, which expresses nontrivial correlations between different colour configurations [51, 52]. However, in our case, the coefficients can be expressed in terms of simple colour factors since we only require virtual amplitudes with up to three coloured particles $(H \rightarrow b \bar{b}$ and $H \rightarrow b \bar{b} g)$. The expansion coefficients $\mathbf{Z}_{b \bar{b}}^{(k)}$ for the $H \rightarrow b \bar{b}$ process are given by

$$
\begin{aligned}
\mathbf{Z}_{b \bar{b}}^{(1)}= & \frac{1}{2 \epsilon}\left(C_{F} \gamma_{\text {cusp }, \mathrm{Q}}^{(0)}\left(v_{23}\right)+2 \gamma_{Q}^{(0)}\right), \\
\mathbf{Z}_{b \bar{b}}^{(2)}= & \frac{1}{8 \epsilon^{2}}\left(C_{F} \gamma_{\text {cusp }, Q}^{(0)}\left(v_{23}\right)+2 \gamma_{Q}^{(0)}\right)\left(C_{F} \gamma_{\text {cusp }, Q}^{(0)}\left(v_{23}\right)+2 \gamma_{Q}^{(0)}-2 \beta_{0}\left(n_{l}\right)\right) \\
& +\frac{1}{4 \epsilon}\left(C_{F} \gamma_{\text {cusp }, Q}^{(1)}\left(v_{23}\right)+2 \gamma_{Q}^{(1)}\right)+\frac{1}{2 \epsilon}\left(C_{F} \gamma_{\text {cusp }, Q}^{(0)}\left(v_{23}\right)+2 \gamma_{Q}^{(0)}\right) \beta_{0, Q} \ln \left(\frac{\mu_{R}^{2}}{m_{b}^{2}}\right),
\end{aligned}
$$


where $\gamma_{\text {cusp }}^{(i)}$ and $\gamma_{\text {cusp, },}^{(0)}\left(v_{23}\right)$ are the massless and massive cusp anomalous dimensions, $v_{23}=\sqrt{1-m_{b}^{4} /\left(q_{2} \cdot q_{3}\right)^{2}}, \beta_{0}\left(n_{l}\right)$ is the zeroth-order coefficient of the QCD $\beta$-function with $n_{l}$ massless flavours, $\beta_{0}\left(n_{l}\right)=\frac{11}{3} C_{A}-\frac{4}{3} T_{F} n_{l}$ and $\beta_{0, Q}=-\frac{4}{3} T_{F}$. The $\gamma_{Q}^{(i)}$ denote the expansion coefficients of the anomalous dimensions of the massive quark and $\mu_{R}$ is the renormalisation scale. We collect the necessary formulae in section B.1. For the $H \rightarrow b \bar{b} g$ process, where we only need the one-loop amplitude, we find

$$
\begin{aligned}
\mathbf{Z}_{b \bar{b} g}^{(1)}=\frac{1}{4 \epsilon^{2}}\left(-C_{A} \gamma_{\text {cusp }}^{(0)}\right)+\frac{1}{2 \epsilon} & {\left[\gamma_{g}^{(0)}+2 \gamma_{Q}^{(0)}+\left(C_{F}-\frac{C_{A}}{2}\right) \gamma_{\text {cusp }, Q}^{(0)}\left(v_{23}\right)\right.} \\
& \left.-\frac{C_{A}}{2} \gamma_{\text {cusp }}^{(0)}\left(\ln \left(\frac{m_{b} \mu_{R}}{2\left(q_{2} \cdot q_{4}\right)}\right)+\ln \left(\frac{m_{b} \mu_{R}}{2\left(q_{3} \cdot q_{4}\right)}\right)+2 i \pi\right)\right]
\end{aligned}
$$

The gluon anomalous dimension $\gamma_{g}^{(0)}$ is also given in section B.1.

\subsection{Phase-space parametrisation}

In this section we outline the parametrisation of the real-emission phase space that we employ throughout the calculation. NNLO corrections to Higgs decays involve contributions with up to two real emissions accompanying the Born process. In our case, the Born process consists of the Higgs boson decaying into massive $b$-quarks $(H \rightarrow b \bar{b})$ and we include final states with one additional gluon $(H \rightarrow b \bar{b} g)$ as well as two additional massless partons $(H \rightarrow b \bar{b} g g$ or $H \rightarrow b \bar{b} q \bar{q}){ }^{1}$

The guiding principle behind the construction outlined in this section is, first, to explicitly parametrise the energies and angles that are responsible for the soft and collinear singularities and, second, to decouple the real-emission phase space from the phase space of the reduced process once a parton becomes unresolved. We note that we work in the Higgs boson rest frame throughout the paper.

The phase-space measure for an emission of a single massless parton in $d=4-2 \epsilon$ space-time dimensions reads

$$
\left[\mathrm{d} q_{i}\right]=\left(\mu_{R}^{2}\right)^{\epsilon} S_{\epsilon} \frac{\mathrm{d}^{d-1} q_{i}}{(2 \pi)^{d-1}\left(2 E_{i}\right)},
$$

where we denote the parton momentum by $q_{i}$ and its energy by $E_{i}$. Note that we also include a global factor $\left(\mu_{R}^{2}\right)^{\epsilon} S_{\epsilon}$ that originates from the strong coupling renormalisation, see discussion below eq. (A.9). We do not introduce an upper bound on the energy of the emitted gluon since it naturally appears due to the energy-momentum conserving $\delta$ function once the measure in eq. (2.16) is considered as a part of a specific process.

Single-emission phase space. We start with the process

$$
H\left(q_{1}\right) \longrightarrow b\left(q_{2}\right)+\bar{b}\left(q_{3}\right)+g\left(q_{4}\right)
$$

and discuss its phase-space parametrisation.

\footnotetext{
${ }^{1}$ The $H \rightarrow b \bar{b} b \bar{b}$ contribution is finite. Thus, it can be integrated using standard techniques and we do not discuss it here.
} 
The phase-space measure reads

$$
\begin{aligned}
\int \mathrm{d} \Phi_{b \bar{b} g}\left(q_{1}\right) & =\int\left[\mathrm{d} q_{4}\right] \int \mathrm{d} \Phi_{b \bar{b}}\left(q_{1}-q_{4}\right) \\
& =\left(\mu_{R}^{2}\right)^{\epsilon} S_{\epsilon} \int \frac{\mathrm{d}^{d-2} \hat{q}_{4}}{2(2 \pi)^{d-1}} \int \mathrm{d} E_{4}\left(E_{4}\right)^{d-3} \int \mathrm{d} \Phi_{b \bar{b}}\left(q_{1}-q_{4}\right),
\end{aligned}
$$

where $\mathrm{d} \Phi_{b \bar{b}}(Q)$ stands for the Born phase space of the two $b$-quarks with total momentum $Q$, and $\hat{q}_{4}$ determines the direction of the gluon momentum,

$$
\hat{q}_{4}^{\mu}=q_{4}^{\mu} / E_{4}
$$

We further parametrise the gluon energy as

$$
E_{4}=E_{\max } \xi_{1}
$$

with $E_{\max }=\frac{1}{2} \beta^{2} M_{H}$ and $\beta=\sqrt{1-4 m_{b}^{2} / M_{H}^{2}}$. This finally leads us to

$$
\begin{aligned}
\int \mathrm{d} \Phi_{b \bar{b} g}\left(q_{1}\right)= & 2^{1-2 \epsilon} E_{\max }^{2}\left(\frac{\mu_{R}^{2}}{E_{\max }^{2}}\right)^{\epsilon} S_{\epsilon} \int \frac{\mathrm{d} \Omega_{4}^{(2-2 \epsilon)}}{2(2 \pi)^{3-2 \epsilon}} \\
& \times \int_{0}^{1} \frac{\mathrm{d} \eta}{(\eta(1-\eta))^{\epsilon}} \int_{0}^{1} \mathrm{~d} \xi_{1} \xi_{1}^{1-2 \epsilon} \int \mathrm{d} \Phi_{b \bar{b}}\left(q_{1}-q_{4}\right) .
\end{aligned}
$$

The $\mathrm{d} \Omega_{4}$ element denotes the angular integral over the direction of $\hat{q}_{4}$. The remaining angular integrals can be performed using

$$
\int \mathrm{d} \Omega^{(a)}=\frac{2 \pi^{a / 2}}{\Gamma(a / 2)} .
$$

For a single gluon emission, the only unresolved limit is the single-soft one, i.e. $\xi_{1} \rightarrow 0$. Obviously, this removes $q_{4}$ from the overall momentum conservation and the integration over the unresolved phase space of $q_{4}$ decouples from the Born phase space. Thus, in that limit we just replace $\int \mathrm{d} \Phi_{b \bar{b}}\left(q_{1}-q_{4}\right)$ by $\int \mathrm{d} \Phi_{b \bar{b}}\left(q_{1}\right)$ in eq. (2.21).

Double-emission phase space. We now focus on the parametrisation of the $H \rightarrow b \bar{b} g g$ phase space. Note that, since we consider $b$-quarks to be massive, there are no singularities associated with kinematic configurations where gluons become collinear to $b$-quarks. Therefore, we do not need to partition the phase space into subsectors, which are usually necessary to disentangle the collinear singularities. Instead, we work with a global parametrisation. In this section we focus on the two-gluon emission case since the parametrisation of the $q \bar{q}$ emission phase space is nearly identical. We comment on the differences where necessary.

We consider the process

$$
H\left(q_{1}\right) \longrightarrow b\left(q_{2}\right)+\bar{b}\left(q_{3}\right)+g\left(q_{4}\right)+g\left(q_{5}\right)
$$


We denote the sum of the gluon momenta by $q_{45}=q_{4}+q_{5}$. The phase space measure then reads

$$
\begin{aligned}
\int \mathrm{d} \Phi_{b \bar{b} g g}\left(q_{1}\right)= & \int\left[\mathrm{d} q_{4}\right] \int\left[\mathrm{d} q_{5}\right] \int \mathrm{d} \Phi_{b \bar{b}}\left(q_{1}-q_{45}\right) \\
= & \left(\mu_{R}^{2}\right)^{2 \epsilon} S_{\epsilon}^{2} \int \frac{\mathrm{d}^{d-2} \hat{q}_{4}}{2(2 \pi)^{d-1}} \int \frac{\mathrm{d}^{d-2} \hat{q}_{5}}{2(2 \pi)^{d-1}} \\
& \times \int \mathrm{d} E_{4}\left(E_{4}\right)^{d-3} \int \mathrm{d} E_{5}\left(E_{5}\right)^{d-3} \int \mathrm{d} \Phi_{b \bar{b}}\left(q_{1}-q_{45}\right),
\end{aligned}
$$

where the vectors $\hat{q}_{4}$ and $\hat{q}_{5}$ determine the directions of the two gluons and the limits of the energy integrals are so that the whole phase space is covered.

It is convenient to introduce an energy ordering among the gluons by partitioning the phase space via

$$
1=\Theta\left(E_{4}-E_{5}\right)+\Theta\left(E_{5}-E_{4}\right)
$$

which leads to the split

$$
\int \mathrm{d} \Phi_{b \bar{b} g g}\left(q_{1}\right)=\int \mathrm{d} \Phi_{b \bar{b} g g}^{E_{4}>E_{5}}\left(q_{1}\right)+\int \mathrm{d} \Phi_{b \bar{b} g g}^{E_{5}>E_{4}}\left(q_{1}\right) .
$$

Throughout the article, we describe calculations only for the region with $E_{4}>E_{5}$; the other region can easily be covered by performing the same steps with the gluon momenta swapped, $q_{4} \leftrightarrow q_{5} .{ }^{2}$ Hence, we parametrise the gluon energies as [32]

$$
E_{4}=E_{45, \max } \xi_{1}\left(1-\frac{\xi_{2}}{2}\right), \quad E_{5}=E_{45, \max } \xi_{1} \frac{\xi_{2}}{2}
$$

where $E_{45, \max }$ is to be chosen such that the integration ranges $\xi_{1} \in[0,1]$ and $\xi_{2} \in[0,1]$ span the whole phase space. Using momentum conservation and considering a configuration where the two $b$-quarks are produced at threshold, we obtain

$$
E_{45, \text { max }}=\frac{M_{H} \beta^{2}}{1+\sqrt{1-\beta^{2} \bar{q}_{45}^{2}}},
$$

where $\bar{q}_{45}=q_{45} / q_{45}^{0}$, which is only a light-like momentum when $q_{5}$ is soft or $q_{4}$ and $q_{5}$ are collinear. In this way, we effectively parametrise the sum of the gluon energies $\left(\xi_{1}=\right.$ $\left.E_{45} / E_{45, \max }\right)$ and their ratio $\left(\xi_{2}=2 E_{5} / E_{45}\right)$.

We also explicitly parametrise the angle $\theta_{45}$ between the two gluons as follows

$$
\eta=\frac{1}{2}\left(1-\cos \theta_{45}\right) \text {. }
$$

The first step in the phase-space construction is to choose a direction for $\hat{q}_{4}$. Here, we explicitly parametrise angle $\theta_{4}$ between the emission and the $\hat{z}$-axis. Next, we fix the

\footnotetext{
${ }^{2}$ Note that thanks to the symmetry between the gluons we have $\int \mathrm{d} \Phi_{b \bar{b} g g}\left(q_{1}\right)=2 \int \mathrm{d} \Phi_{b \bar{b} g g}^{E_{4}>E_{5}}\left(q_{1}\right)$. However when considering a $q \bar{q}$ emission such a simplification may only be used if the symmetry $q \leftrightarrow \bar{q}$ also holds for the observable under consideration. Otherwise the two parts of the phase space, introduced in eq. (2.26), have to be considered separately.
} 
direction of $\hat{q}_{5}$ relative to $\hat{q}_{4}$ using the angle $\theta_{45}$ between them and the angle $\phi$ which is the azimuthal angle of $\hat{q}_{5}$ around the direction of $\hat{q}_{4}$. Given the directions $\hat{q}_{4}$ and $\hat{q}_{5}$ as well as $\xi_{2}$, we calculate the vector $\bar{q}_{45}$ via

$$
\bar{q}_{45}=\left(1-\frac{\xi_{2}}{2}\right) \hat{q}_{4}+\frac{\xi_{2}}{2} \hat{q}_{5} .
$$

This is sufficient to use eq. (2.28) in order to calculate the upper bound on the energy and from that also the individual energies $E_{4}$ and $E_{5}$, which fully determines $q_{4}$ and $q_{5}$. Then we generate a Born phase-space configuration with invariant mass $Q^{2}=\left(q_{1}-q_{45}\right)^{2}$ in its rest frame; this is a back-to-back configuration of the two $b$-quarks. Finally, we boost the Born configuration to have total momentum $Q=q_{1}-q_{45}$ in the Higgs rest frame in order to restore momentum conservation. The corresponding phase-space measure reads

$$
\begin{aligned}
\int \mathrm{d} \Phi_{b \bar{b} g g}^{E_{4}>E_{5}}\left(q_{1}\right)=2^{2 \epsilon-2}\left(\mu_{R}^{2}\right)^{2 \epsilon} S_{\epsilon}^{2} \\
\quad \times \int \frac{\mathrm{d} \Omega_{4}^{(2-2 \epsilon)}}{2(2 \pi)^{3-2 \epsilon}} \int_{0}^{\pi} \mathrm{d} \theta_{4}\left(\sin \theta_{4}\right)^{1-2 \epsilon} \int \frac{\mathrm{d} \Omega_{5}^{(1-2 \epsilon)}}{2(2 \pi)^{3-2 \epsilon}} \int_{0}^{1} \frac{\mathrm{d} \eta}{\eta^{\epsilon}(1-\eta)^{\epsilon}} \int_{0}^{\pi} \mathrm{d} \phi(\sin \phi)^{-2 \epsilon} \\
\quad \times \int_{0}^{1} \mathrm{~d} \xi_{1} \int_{0}^{1} \mathrm{~d} \xi_{2} E_{45, \max }^{4-4 \epsilon} \xi_{1}^{3-4 \epsilon} \xi_{2}^{1-2 \epsilon}\left(2-\xi_{2}\right)^{1-2 \epsilon} \int \mathrm{d} \Phi_{b \bar{b}}\left(q_{1}-q_{45}\right) .
\end{aligned}
$$

The unparametrised angles in $\mathrm{d} \Omega_{4}$ and $\mathrm{d} \Omega_{5}$ can be integrated in the end using eq. (2.22).

The parametrisation shown in eq. (2.31) achieves the desired decoupling of the emission phase space in unresolved limits. In the collinear $(\eta \rightarrow 0)$ and single-soft $\left(\xi_{2} \rightarrow 0\right)$ limits the energy bound $E_{45, \max }$ simplifies to $E_{\max }=\frac{1}{2} \beta^{2} M_{H}$ and becomes independent of $\eta$ and $\xi_{2}$. In the single-soft limit the momentum $q_{5}$ decouples from the energy-momentum conserving $\delta$-function and the integrations over $\eta$ and $\xi_{2}$ decouple from the resolved phase space. In the collinear limit the momentum conservation depends only on the sum of momenta $q_{45}$, which is the on-shell momentum of the massless parent parton of the splitting and is independent of $\xi_{2}$. Again, the integrations over $\eta$ and $\xi_{2}$ decouple from the resolved phase space. Note that the collinear and the single-soft limits both yield the same resolved configuration. Furthermore, in the double-soft limit, both gluons decouple from the momentum conservation and the integrals over $\xi_{1}, \xi_{2}$ and $\eta$ can be carried out for a fixed Born configuration.

\subsection{Pole vs. $\overline{\text { MS }}$ Yukawa coupling}

Already in the first calculation of the radiative corrections to the Higgs boson decay rate to fermions discussed in ref. [4], it has been recognised that the result expressed in terms of the on-shell Yukawa coupling contains large logarithms in the limit $m_{b} / M_{H} \ll 1$. It has also been shown there that these large logarithms can be avoided by reexpressing the result in terms of the $\overline{\mathrm{MS}}$ Yukawa coupling evaluated at the renormalisation scale $\mu=M_{H}$.

The Yukawa coupling in the $\overline{\mathrm{MS}}$ scheme $\bar{y}_{b}(\mu)$ is related to the $\overline{\mathrm{MS}}$ mass $\bar{m}_{b}(\mu)$ via

$$
\bar{y}_{b}(\mu)=\left(2 \sqrt{2} G_{F}\right)^{1 / 2} \bar{m}_{b}(\mu),
$$

where $\mu$ is the renormalisation scale. Thus, the relation between the on-shell and $\overline{\mathrm{MS}}$ Yukawa couplings can be deduced from the corresponding relation between the masses, 
which we need up to $\mathcal{O}\left(\alpha_{s}^{2}\right)[53,54]$. It reads

$$
y_{b}^{2}=\bar{y}_{b}^{2}(\mu)\left[1+\left(\frac{\alpha_{s}(\mu)}{\pi}\right) r_{1}\left(m_{b}, \mu\right)+\left(\frac{\alpha_{s}(\mu)}{\pi}\right)^{2} r_{2}\left(m_{b}, \mu\right)+\mathcal{O}\left(\alpha_{s}^{3}\right)\right] \text {, }
$$

where the coefficients $r_{i}\left(m_{b}, \mu\right)$ are presented in section B.2.

Note that we only reexpress the overall Yukawa coupling in this way, but we keep the mass dependence of the matrix elements and kinematical invariants in terms of the pole mass, similar to ref. [21].

The total decay width and its expansion coefficients computed with the $\overline{\mathrm{MS}}$ Yukawa coupling are denoted with a bar, i.e.

$$
\bar{\Gamma}^{b \bar{b}}=\bar{\Gamma}_{\mathrm{LO}}^{b \bar{b}}\left[1+\left(\frac{\alpha_{s}}{\pi}\right) \bar{\gamma}_{1}^{b \bar{b}}+\left(\frac{\alpha_{s}}{\pi}\right)^{2} \bar{\gamma}_{2}^{b \bar{b}}+\mathcal{O}\left(\alpha_{s}^{3}\right)\right]
$$

where the expansion coefficients in the two schemes are related by

$$
\begin{aligned}
& \bar{\gamma}_{1}^{b \bar{b}}=\gamma_{1}^{b \bar{b}}+r_{1}, \\
& \bar{\gamma}_{2}^{b \bar{b}}=\gamma_{2}^{b \bar{b}}+r_{1} \gamma_{1}^{b \bar{b}}+r_{2} .
\end{aligned}
$$

As discussed before, the large logarithmic corrections to the total decay width can be mitigated by reexpressing the result in terms of the running $\overline{\mathrm{MS}}$ mass of the $b$-quark. However, in a fully differential calculation these logarithms partially enter through corrections related to real emissions. In this case, they arise during phase-space integration of the emission. A priori, since the mass of the $b$-quark is small compared to the Higgs mass, one could be worried about possible numerical instabilities when working with a fully differential calculation. However, it turns out that in our implementation they do not pose serious numerical problems.

\section{$3 \quad H \rightarrow b \bar{b}$ decay at NLO}

In this section we briefly describe the calculation of the NLO QCD corrections to the $H \rightarrow b \bar{b}$ decay with massive $b$-quarks. Although such a calculation is straightforward, we find it useful to review it in order to clarify our notation and conventions. At this order of perturbation theory we need to consider real (R) and virtual corrections (V).

Nowadays the fully differential treatment of this decay mode can easily be obtained using the FKS $[55,56]$ or Catani-Seymour $[52,57,58]$ subtraction schemes. At NLO, our approach is essentially equivalent to the FKS subtraction method.

\subsection{Real contribution}

At NLO we consider one real emission in addition to the Born process, which means that we need to integrate the function

$$
F_{L M}(b \bar{b} g)=\mathrm{d} \Phi_{b \bar{b} g}\left(q_{1}\right)\left|\mathcal{M}_{b \bar{b} g}^{(0)}\right|^{2} \mathcal{F}_{\text {kin }}(b \bar{b} g)
$$


over the $b \bar{b} g$ phase space. This integral is divergent in four dimensions, due to the soft singularity of the gluon. However, there are no collinear singularities since they are regulated by the $b$-quark mass.

We define a projection operator that allows us to extract the soft divergence and to regulate the limit. Given a quantity $A$ that depends on the momenta, we define a projection operator for the soft limit of momentum $q_{4}$ as

$$
S_{4} A=\lim _{\xi_{1} \rightarrow 0} A,
$$

where $\xi_{1}$ refers to the parametrisation of eq. (2.21). We define the operator to act on all quantities to the right of the $S_{4}$ symbol, extracting the leading asymptotic behaviour in $\xi_{1}$ of the quantity $A$ if the actual limit does not exist.

Denoting the identity operation by $I$, we can immediately write

$$
F_{L M}(b \bar{b} g)=\left(I-S_{4}\right) F_{L M}(b \bar{b} g)+S_{4} F_{L M}(b \bar{b} g),
$$

where the first term is now regularised in the soft limit and can be integrated in four dimensions. The soft singularity is exposed in the second term in eq. (3.3), which therefore needs to be evaluated in $d$ dimensions. Once the soft limit is taken, the only remaining dependence on $\xi_{1}$ in $S_{4} F_{L M}(b \bar{b} g)$ is the leading behaviour $\xi_{1}^{-1-2 \epsilon}$. Thus, the $\xi_{1}$ integration becomes trivial and the soft singularity manifests itself as an explicit $\epsilon^{-1}$ pole.

In order to show pole cancellation pointwise in the Born phase space, we split the real emission contribution into two parts. The finite contribution is given by

$$
\begin{aligned}
2 M_{H}\left\langle\mathrm{~d} \Gamma_{\mathrm{R}}^{\mathrm{F}}(b \bar{b} g)\right\rangle & =\left\langle\left(I-S_{4}\right) F_{L M}(b \bar{b} g)\right\rangle \\
& =\left\langle F_{L M}(b \bar{b} g)+g_{s}^{2} C_{F}\left(\mathcal{S}_{22,4}^{(0)}-2 \mathcal{S}_{23,4}^{(0)}+\mathcal{S}_{33,4}^{(0)}\right) S_{4}\left[\mathrm{~d} q_{4}\right] F_{L M}(b \bar{b})\right\rangle .
\end{aligned}
$$

Here, we use the factorisation formula for the soft limit as discussed in section C.2, where also the eikonal factors $\mathcal{S}_{i j, k}^{(0)}$ are defined. Moreover, we have the unresolved contribution, which contains the integrated subtraction term and reads

$$
\begin{aligned}
2 M_{H}\left\langle\mathrm{~d} \Gamma_{\mathrm{R}}^{\mathrm{U}}(b \bar{b} g)\right\rangle & =\left\langle S_{4} F_{L M}(b \bar{b} g)\right\rangle \\
& =-\left\langle g_{s}^{2} C_{F}\left(\mathcal{S}_{22, \text { int }}^{(0)}-2 \mathcal{S}_{23, \text { int }}^{(0)}+\mathcal{S}_{33, \text { int }}^{(0)}\right) F_{L M}(b \bar{b})\right\rangle,
\end{aligned}
$$

with the integrated eikonal factors $\mathcal{S}_{i j \text {,int }}^{(0)}$ given in section D.2. Here, the integral over the unresolved phase space of the gluon was performed and we are only left with the phasespace integral over the underlying Born process.

\subsection{Virtual contribution}

For the virtual contribution, the phase-space integration is the same as that for the Born process, but we need to consider a one-loop virtual amplitude. Although this amplitude has an $\epsilon^{-1}$ pole, the singular part can be written as a product of a tree-level matrix element and a kinematics-dependent coefficient, as indicated in section 2.3. We have

$$
2 \operatorname{Re}\left\langle\mathcal{M}_{b \bar{b}}^{(0)} \mid \mathcal{M}_{b \bar{b}}^{(1)}\right\rangle=2 \operatorname{Re}\left\langle\mathcal{M}_{b \bar{b}}^{(0)} \mid \mathcal{F}_{b \bar{b}}^{(1)}\right\rangle+2 \operatorname{Re}\left(\mathbf{Z}_{b \bar{b}}^{(1)}\right)\left\langle\mathcal{M}_{b \bar{b}}^{(0)} \mid \mathcal{M}_{b \bar{b}}^{(0)}\right\rangle
$$


where the term with the $\mathbf{Z}$ operator contains an explicit $\epsilon^{-1}$ pole, while the second term is finite. As a shorthand we introduce

$$
F_{L V}^{\mathrm{fin}}(b \bar{b})=\mathrm{d} \Phi_{b \bar{b}}\left(q_{1}\right)\left(\frac{\alpha_{s}}{4 \pi}\right) 2 \operatorname{Re}\left\langle\mathcal{M}_{b \bar{b}}^{(0)} \mid \mathcal{F}_{b \bar{b}}^{(1)}\right\rangle \mathcal{F}_{\text {kin }}(b \bar{b}) .
$$

Accordingly, we define two contributions to the virtual correction: the virtual finite contribution

$$
2 M_{H}\left\langle\mathrm{~d} \Gamma_{\mathrm{V}}^{\mathrm{F}}(b \bar{b})\right\rangle=\left\langle F_{L V}^{\mathrm{fin}}(b \bar{b})\right\rangle
$$

and the virtual unresolved contribution

$$
2 M_{H}\left\langle\mathrm{~d} \Gamma_{\mathrm{V}}^{\mathrm{U}}(b \bar{b})\right\rangle=\left\langle\left(\frac{\alpha_{s}}{4 \pi}\right) 2 \operatorname{Re}\left(\mathbf{Z}_{b \bar{b}}^{(1)}\right) F_{L M}(b \bar{b})\right\rangle .
$$

The expansion coefficients of the $\mathbf{Z}$ operator are given in eq. (2.13).

\subsection{Pole cancellation}

At this point we can combine all contributions that enter the NLO calculation. We remind the reader that the $\mathrm{d} \Gamma_{\mathrm{R}}^{\mathrm{F}}(b \bar{b} g)$ and $\mathrm{d} \Gamma_{\mathrm{V}}^{\mathrm{F}}(b \bar{b})$ terms are free of $\epsilon^{-1}$ poles, while the $\mathrm{d} \Gamma_{\mathrm{R}}^{\mathrm{U}}(b \bar{b} g)$ and $\mathrm{d} \Gamma_{\mathrm{V}}^{\mathrm{U}}(b \bar{b})$ terms feature $\epsilon^{-1}$ poles.

Expanding the explicit results for the real unresolved contribution from eq. (3.5) up to $\mathcal{O}\left(\epsilon^{-1}\right)$ we find

$$
2 M_{H}\left\langle\mathrm{~d} \Gamma_{\mathrm{R}}^{\mathrm{U}}(b \bar{b} g)\right\rangle=\frac{1}{\epsilon}\left[\left(\frac{\alpha_{s}}{4 \pi}\right) 4 C_{F}\left[1+\frac{1+\beta^{2}}{2 \beta} \log \left(\frac{1-\beta}{1+\beta}\right)\right]\left\langle F_{L M}(b \bar{b})\right\rangle\right]+\mathcal{O}\left(\epsilon^{0}\right) .
$$

Analogously, the virtual unresolved contribution, defined in eq. (3.9), yields

$$
2 M_{H}\left\langle\mathrm{~d} \Gamma_{\mathrm{V}}^{\mathrm{U}}(b \bar{b})\right\rangle=-\frac{1}{\epsilon}\left[\left(\frac{\alpha_{s}}{4 \pi}\right) 4 C_{F}\left[1+\frac{1+\beta^{2}}{2 \beta} \log \left(\frac{1-\beta}{1+\beta}\right)\right]\left\langle F_{L M}(b \bar{b})\right\rangle\right] .
$$

Note that the constant term in $\epsilon$ is absent since our definition of the $\mathbf{Z}$ operator of eq. (2.8) includes only $\epsilon^{-1}$ poles.

Obviously, the poles of the two contributions cancel. A crucial part of the argument is to notice that the $\mathrm{d} \Gamma_{\mathrm{R}}^{\mathrm{U}}(b \bar{b} g)$ part, after integrating out the real emission, is a function of a Born-like phase-space configuration, as is the $\mathrm{d} \Gamma_{\mathrm{V}}^{\mathrm{U}}(b \bar{b})$ contribution. This allows us to demonstrate the pole cancellation for any point of the $\mathrm{d} \Phi_{b \bar{b}}$ phase space and without specifying the explicit form of the LO matrix element.

\section{$4 \quad H \rightarrow b \bar{b}$ decay at NNLO}

We now consider the NNLO QCD corrections to the $H \rightarrow b \bar{b}$ process keeping the full dependence on the $b$-quark mass. To this end, we need to consider several contributions including

- the double-real contribution (RR) - where the leading-order decay is accompanied by an emission of a pair of massless partons $(H \rightarrow b \bar{b} g g$ and $H \rightarrow b \bar{b} q \bar{q})$ or an additional pair of $b$-quarks $(H \rightarrow b \bar{b} b \bar{b})$; 
- the real-virtual contribution (RV) - where we consider one-loop virtual corrections to the process $H \rightarrow b \bar{b} g$;

- the double-virtual contribution (VV) - where we consider two-loop virtual corrections to the Born process $H \rightarrow b \bar{b}$.

Except for the $H \rightarrow b \bar{b} b \bar{b}$ subprocess, all these contributions are divergent in four dimensions, due to soft and collinear singularities. For that reason, we follow the general method recapitulated in section 2.2 and adopt a subtraction scheme that allows us to regulate all singular limits and treat the divergent integrals analytically in $d=4-2 \epsilon$ dimensions.

Apart from these divergent contributions we distinguish the subprocess $H \rightarrow b \bar{b} b \bar{b}$ which enters the calculation at $\mathcal{O}\left(\alpha_{s}^{2}\right)$. Indeed, this subprocess is finite in four dimensions because of the non-zero $b$-quark mass. Hence, it does not require any regularisation. It is calculated by directly integrating the squared tree-level amplitude over the phase space $\mathrm{d} \Phi_{b \bar{b} b \bar{b}}$. In our implementation we use the sequential algorithm [59] to generate kinematic configurations and the phase-space measure.

Finally, a distinct class of corrections to the $H \rightarrow b \bar{b}$ decay that appears at second order of perturbation theory is related to Feynman diagrams where the $H \rightarrow b \bar{b}$ or $H \rightarrow b \bar{b} g$ transition is induced by the Higgs boson coupling to gluons via a top-quark loop. This contribution is finite on its own and, hence, can be studied separately — we defer the discussion of these top-quark mediated corrections to section 4.5.

All tree-level amplitudes that we use in this paper are calculated using the spinorhelicity formalism. ${ }^{3}$ The treatment of massive external particles follows along the lines of appendix A of ref. [61]. The one-loop amplitudes are calculated using a combination of Passarino-Veltman reduction [62], to express them through one-loop scalar integrals, and spinor-helicity techniques, to treat spinor structures appearing in the amplitudes. The one-loop scalar integrals are evaluated using the library QCDLoop [63, 64]. We assemble the two-loop using the two-loop scalar heavy-quark form factor from ref. [65]; equivalent results can be obtained using the expressions from ref. [66]. The form factor is expressed in terms of harmonic polylogarithms [67], which we evaluate using HPLOG [68].

It is useful to stress that we show cancellation of all $\epsilon^{-1}$ poles without referring to the specific form of the matrix elements. The expressions for all amplitudes are only needed to calculate finite corrections to the considered process and, hence, we restrict ourselves to the construction of only four-dimensional matrix elements.

\subsection{Double-real contribution}

In this section we only focus on the $H \rightarrow b \bar{b} g g$ and $H \rightarrow b \bar{b} q \bar{q}$ subprocesses. The $H \rightarrow b \bar{b} b \bar{b}$ process is completely finite on its own and does not require any regularisation procedure. For the record we write

$$
2 M_{H}\left\langle\mathrm{~d} \Gamma_{b \bar{b} b \bar{b}}\right\rangle=\left\langle F_{L M}(b \bar{b} b \bar{b})\right\rangle,
$$

where the shorthand $F_{L M}(b \bar{b} b \bar{b})$ is introduced in eq. (2.5).

\footnotetext{
${ }^{3}$ For a review, see e.g. ref. [60].
} 
Thanks to the non-zero $b$-quark mass, the singularity structure of the double-real contribution is simple. Indeed, we only need to take into account three possible limits:

- the soft limit $\left(S_{5}\right)$ - where the energy of one of the partons vanishes, i.e. $\xi_{2} \rightarrow 0$;

- the double-soft limit $\left(S_{45}\right)$ - where the energies of both additional partons vanish at a similar rate, i.e. $\xi_{1} \rightarrow 0$;

- the collinear limit $\left(C_{45}\right)$ - where the momenta of the two additional partons become collinear to each other, i.e. $\eta \rightarrow 0$.

The variables $\xi_{1}, \xi_{2}$ and $\eta$ refer to the parametrisation introduced in eq. (2.31).

We define projection operators that allow us to extract divergences in each of the singular regions. Given a quantity $A$ which depends on the momenta of the $b$-quarks and gluons, we define the action of the projection operators as follows

$$
S_{45} A=\lim _{\xi_{1} \rightarrow 0} A, \quad S_{5} A=\lim _{\xi_{2} \rightarrow 0} A, \quad C_{45} A=\lim _{\eta \rightarrow 0} A .
$$

Again, we note that taking limits in eq. (4.2) should be understood as extracting the most singular part of the quantity $A$ in a particular limit whenever the limit in the conventional sense does not exist.

With these operators, we construct a nested subtraction formula which extracts all singularities of the double-real contribution,

$$
\begin{aligned}
F_{L M}(b \bar{b} g g)= & \left(I-S_{45}\right) F_{L M}(b \bar{b} g g)+S_{45} F_{L M}(b \bar{b} g g) \\
= & \left(I-S_{5}\right)\left(I-S_{45}\right) F_{L M}(b \bar{b} g g)+S_{5}\left(I-S_{45}\right) F_{L M}(b \bar{b} g g)+S_{45} F_{L M}(b \bar{b} g g) \\
= & \left(I-S_{5}\right)\left(I-S_{45}\right)\left(I-C_{45}\right) F_{L M}(b \bar{b} g g)+S_{5}\left(I-S_{45}\right)\left(I-C_{45}\right) F_{L M}(b \bar{b} g g) \\
& +\left(I-S_{5}\right)\left(I-S_{45}\right) C_{45} F_{L M}(b \bar{b} g g)+S_{5}\left(I-S_{45}\right) C_{45} F_{L M}(b \bar{b} g g) \\
& +S_{45} F_{L M}(b \bar{b} g g) .
\end{aligned}
$$

To derive eq. (4.3), we start by regularising the double-soft singularity $\left(S_{45}\right)$, followed by further regularisation of the single-soft limit $\left(S_{5}\right)$. In the last step we introduce a subtraction term for the collinear singularity $\left(C_{45}\right)$. Moreover, as these operators commute with each other [26], we have a freedom to choose which limit to take first. Note that in eq. (4.3) we start with the collinear limit, where appropriate, having in mind the simplicity of the corresponding factorisation formulae.

We subdivide eq. (4.3) into separate contributions according to the final state multiplicity. This allows us to discuss pole cancellation for each of these contributions separately.

The first term on the right-hand side of eq. (4.3) represents the fully regulated doublereal contribution. Therefore, it can be evaluated in four dimensions using standard numerical techniques. We write

$$
2 M_{H}\left\langle\mathrm{~d} \Gamma_{\mathrm{RR}}^{\mathrm{F}}(b \bar{b} g g)\right\rangle=\left\langle\left(I-S_{5}\right)\left(I-S_{45}\right)\left(I-C_{45}\right) F_{L M}(b \bar{b} g g)\right\rangle,
$$

and similarly for the $q \bar{q}$ emission

$$
2 M_{H}\left\langle\mathrm{~d} \Gamma_{\mathrm{RR}}^{\mathrm{F}}(b \bar{b} q \bar{q})\right\rangle=n_{l}\left\langle\left(I-S_{45}\right)\left(I-C_{45}\right) F_{L M}(b \bar{b} q \bar{q})\right\rangle+(q \leftrightarrow \bar{q}),
$$


where $n_{l}$ is the number of massless quark flavours and the last term corresponds to the phase-space region with $E_{5}>E_{4}$. Note that in case of the $q \bar{q}$ emission we do not subtract the single-soft limit $\left(S_{5}\right)$ since this limit is not singular in case of a $g \rightarrow q \bar{q}$ splitting. The explicit form of the subtraction terms generated by the limit operators can be constructed using the factorisation formulae collected in section C.

The next three terms on the right-hand side of eq. (4.3) are regulated in the double-soft limit, but they are evaluated in at least one of the other two limits $\left(C_{45}\right.$ or $\left.S_{5}\right)$. As this leaves one of the real emissions unresolved, we call this contribution single-unresolved. Since we perform the integral over $\left[\mathrm{d} q_{5}\right]$ analytically, we do not need to further regulate the $S_{5}$ and $C_{45}$ limits. Thus, we rearrange these terms such that for the gluon emissions they read

$$
\begin{aligned}
2 M_{H} \mathrm{~d} \Gamma_{\mathrm{RR}}^{\mathrm{SU}}(b \bar{b} g g)= & \left\langle\left( S_{5}\left(I-S_{45}\right)\left(I-C_{45}\right)+\left(I-S_{5}\right)\left(I-S_{45}\right) C_{45}\right.\right. \\
& \left.\left.+S_{5}\left(I-S_{45}\right) C_{45}\right) F_{L M}(b \bar{b} g g)\right\rangle \\
= & \left\langle\left(S_{5}\left(I-S_{45}\right)+\left(I-S_{45}\right) C_{45}-S_{5}\left(I-S_{45}\right) C_{45}\right) F_{L M}(b \bar{b} g g)\right\rangle \\
= & \left\langleg _ { s } ^ { 2 } \left(\mathcal{P}_{g g, \text { int }}^{(0)}-\mathcal{P}_{g g, \text { int }}^{(0), \text { oft }}+C_{A}\left(\mathcal{S}_{24, \text { int }}^{(0)}+\mathcal{S}_{34, \text { int }}^{(0)}-\mathcal{S}_{23, \text { int }}^{(0)}\right)\right.\right. \\
& \left.\left.-C_{F}\left(\mathcal{S}_{22, \text { int }}^{(0)}-2 \mathcal{S}_{23, \text { int }}^{(0)}+\mathcal{S}_{33, \text { int }}^{(0)}\right)\right)\left(\xi_{1} / 2\right)^{-2 \epsilon}\left(I-S_{4}\right) F_{L M}(b \bar{b} g)\right\rangle .
\end{aligned}
$$

For the $q \bar{q}$ emission, we obtain

$$
\begin{aligned}
2 M_{H}\left\langle\mathrm{~d} \Gamma_{\mathrm{RR}}^{\mathrm{SU}}(b \bar{b} q \bar{q})\right\rangle & =2 n_{l}\left\langle\left(I-S_{45}\right) C_{45} F_{L M}(b \bar{b} q \bar{q})\right\rangle \\
& =2 n_{l}\left\langle g_{s}^{2} \mathcal{P}_{q \bar{q}, \text { int }}^{(0)}\left(\xi_{1} / 2\right)^{-2 \epsilon}\left(I-S_{4}\right) F_{L M}(b \bar{b} g)\right\rangle
\end{aligned}
$$

where we use the fact that in the collinear limit any infrared-safe observable must be symmetric under $q \leftrightarrow \bar{q}$, which leads to the factor 2 in eq. (4.7). To get from the first to the second lines of eqs. (4.6) and (4.7), we use the factorisation formulae given in section $\mathrm{C}$ and integrate them over the unresolved phase space $\left[\mathrm{d} q_{5}\right]$, which yields the integrated splitting functions $\mathcal{P}_{i j \text {,int }}^{(0)}$ and the integrated eikonal factors $\mathcal{S}_{i j \text {,int }}^{(0)}$. Their calculation is described given in section $\mathrm{D}$. The soft-regulated matrix element $\left(I-S_{4}\right) F_{L M}(b \bar{b} g)$ has the same form as the real finite contribution at NLO, eq. (3.4).

Finally, the last term in eq. (4.3) is evaluated in the double-soft limit. In this case, both partons are unresolved and we call this contribution double-unresolved. We find

$$
\begin{aligned}
2 M_{H}\left\langle\mathrm{~d} \Gamma_{\mathrm{RR}}^{\mathrm{DU}}(b \bar{b} g g)\right\rangle & =\left\langle S_{45} F_{L M}(b \bar{b} g g)\right\rangle \\
& =\left\langle g_{s}^{4} \operatorname{DSoft}_{g g, \text { int }}^{(0)}\left(q_{2}, q_{3}\right) F_{L M}(b \bar{b})\right\rangle, \\
2 M_{H}\left\langle\mathrm{~d} \Gamma_{\mathrm{RR}}^{\mathrm{DU}}(b \bar{b} q \bar{q})\right\rangle & =2 n_{l}\left\langle S_{45} F_{L M}(b \bar{b} q \bar{q})\right\rangle \\
& =2 n_{l}\left\langle g_{s}^{4} \operatorname{DSoft}_{q \bar{q}, \text { int }}^{(0)}\left(q_{2}, q_{3}\right) F_{L M}(b \bar{b})\right\rangle .
\end{aligned}
$$

The double-soft emissions decouple from the hard process and the whole term can be written as a product of the double-soft function and the Born matrix element. After performing the integration over the momenta of the soft partons, we denote the integrated double-soft function as $\mathrm{DSoft}_{i j \text {,int }}^{(0)}\left(q_{2}, q_{3}\right)$. Details of this calculation are presented in section D.4. 


\subsection{Real-virtual contribution}

We need to consider the $H \rightarrow b \bar{b} g$ amplitude at one-loop level and integrate its product with the tree-level amplitude for the $H \rightarrow b \bar{b} g$ process over the phase space $\mathrm{d} \Phi_{b \bar{b} g}$. In the following, we use the shorthand

$$
F_{L V}(b \bar{b} g)=\mathrm{d} \Phi_{b \bar{b} g}\left(q_{1}\right)\left(\frac{\alpha_{s}}{4 \pi}\right) 2 \operatorname{Re}\left\langle\mathcal{M}_{b \bar{b} g}^{(0)} \mid \mathcal{M}_{b \bar{b} g}^{(1)}\right\rangle \mathcal{F}_{\text {kin }}(b \bar{b} g) .
$$

As for the real contribution at NLO, we regulate the soft singularity using the soft limit operator $S_{4}$ defined in eq. (3.2) and find

$$
F_{L V}(b \bar{b} g)=\left(I-S_{4}\right) F_{L V}(b \bar{b} g)+S_{4} F_{L V}(b \bar{b} g) .
$$

We split the one-loop amplitude into a singular part, containing all $\epsilon^{-1}$ poles from loop integrals, and a finite remainder, see section 2.3. We write

$$
2 \operatorname{Re}\left\langle\mathcal{M}_{b \bar{b} g}^{(0)} \mid \mathcal{M}_{b \bar{b} g}^{(1)}\right\rangle=2 \operatorname{Re}\left(\mathbf{Z}_{b \bar{b} g}^{(1)}\right)\left\langle\mathcal{M}_{b \bar{b} g}^{(0)} \mid \mathcal{M}_{b \bar{b} g}^{(0)}\right\rangle+2 \operatorname{Re}\left\langle\mathcal{M}_{b \bar{b} g}^{(0)} \mid \mathcal{F}_{b \bar{b} g}^{(1)}\right\rangle,
$$

where the coefficient of the $\mathbf{Z}$ operator is given in eq. (2.15) and the second term contains the finite remainder of the one-loop amplitude defined in eq. (2.10).

In total, we split the calculation into four contributions, which need to be combined with the corresponding terms from the double-real and double-virtual contributions to show pole cancellation. The first one is given by the soft-regulated terms containing the finite remainder of the $H \rightarrow b \bar{b} g$ matrix element. It is free of $\epsilon^{-1}$ poles and thus we refer to it as the real-virtual finite contribution. It reads

$$
\begin{aligned}
2 M_{H}\left\langle\mathrm{~d} \Gamma_{\mathrm{RV}}^{\mathrm{F}}\right\rangle= & \left\langle\left(I-S_{4}\right) F_{L V}^{\mathrm{fin}}(b \bar{b} g)\right\rangle \\
= & \left\langle F_{L V}^{\mathrm{fin}}(b \bar{b} g)\right. \\
& +g_{s}^{2} C_{F}\left(\mathcal{S}_{22,4}^{(0)}-2 \mathcal{S}_{23,4}^{(0)}+\mathcal{S}_{33,4}^{(0)}\right) \\
& \left.\times\left(S_{4}\left[\mathrm{~d} q_{4}\right] F_{L V}^{\mathrm{fin}}(b \bar{b})+\left(\frac{\alpha_{s}}{4 \pi}\right)\left[\mathcal{R}_{23,4}^{(1)}+Z_{A}^{(1)}+Z_{\alpha_{s}}^{(1)}\right]_{\epsilon^{0}} S_{4}\left[\mathrm{~d} q_{4}\right] F_{L M}(b \bar{b})\right)\right\rangle,
\end{aligned}
$$

where the necessary formulae for the factorisation of the one-loop matrix element in the soft limit are given in section $\mathrm{C}$ and the renormalisation constants $Z_{A}$ and $Z_{\alpha_{s}}$ are given in section A. The notation $[\ldots]_{\epsilon^{0}}$ indicates that the $\mathcal{O}\left(\epsilon^{0}\right)$ term of the expression between the brackets should be taken. We use $F_{L V}^{\mathrm{fin}}(b \bar{b} g)$ in analogy to the definition in eq. (3.7).

The corresponding term containing the soft-regulated $\mathbf{Z}$ operator for the $H \rightarrow b \bar{b} g$ process is called real-virtual single-unresolved contribution and is given by

$$
\begin{aligned}
2 M_{H}\left\langle\mathrm{~d} \Gamma_{\mathrm{RV}}^{\mathrm{SU}}\right\rangle= & \left\langle\left(\frac{\alpha_{s}}{4 \pi}\right)\left(I-S_{4}\right) 2 \operatorname{Re}\left(\mathbf{Z}_{b \bar{b} g}^{(1)}\right) F_{L M}(b \bar{b} g)\right\rangle \\
= & \left\langle\left(\frac{\alpha_{s}}{4 \pi}\right) 2 \operatorname{Re}\left(\mathbf{Z}_{b \bar{b} g}^{(1)}\right) F_{L M}(b \bar{b} g)\right. \\
& +g_{s}^{2}\left(\frac{\alpha_{s}}{4 \pi}\right) C_{F}\left(\mathcal{S}_{22,4}^{(0)}-2 \mathcal{S}_{23,4}^{(0)}+\mathcal{S}_{33,4}^{(0)}\right) \\
& \left.\times\left(\left[\mathcal{R}_{23,4}^{(1)}+Z_{\alpha_{s}}^{(1)}+Z_{A}^{(1)}\right]_{\text {poles }}+2 \operatorname{Re}\left(\mathbf{Z}_{b \bar{b}}^{(1)}\right)\right) S_{4}\left[\mathrm{~d} q_{4}\right] F_{L M}(b \bar{b})\right\rangle,
\end{aligned}
$$

where the subscript "poles" refers to taking only the pole terms up to $\mathcal{O}\left(\epsilon^{-1}\right)$ into account. 
The remaining two contributions are evaluated in the soft limit and are integrated over the unresolved phase space $\left[\mathrm{d} q_{4}\right]$. The integrated subtraction term containing the finite remainder carries the superscript FR and reads

$$
\begin{aligned}
2 M_{H}\left\langle\mathrm{~d} \Gamma_{\mathrm{RV}}^{\mathrm{FR}}\right\rangle & =\left\langle S_{4} F_{L V}^{\mathrm{fin}}(b \bar{b} g)\right\rangle \\
& =-\left\langle g_{s}^{2} C_{F}\left(\mathcal{S}_{22, \text { int }}^{(0)}-2 \mathcal{S}_{23, \text { int }}^{(0)}+\mathcal{S}_{33, \text { int }}^{(0)}\right) F_{L V}^{\mathrm{fin}}(b \bar{b})\right\rangle .
\end{aligned}
$$

Again, the $\mathcal{S}_{i j \text {,int }}^{(0)}$ correspond to integrated eikonal factors discussed in section D.2.

Finally, the contribution containing the soft limit of the $\mathbf{Z}$ operator integrated over the unresolved phase space is referred to as the real-virtual double-unresolved contribution. We obtain

$$
\begin{aligned}
2 M_{H}\left\langle\mathrm{~d} \Gamma_{\mathrm{RV}}^{\mathrm{DU}}\right\rangle=\left\langle\left(\frac{\alpha_{s}}{4 \pi}\right) S_{4} 2 \operatorname{Re}\left(\mathbf{Z}_{b \bar{b} g}^{(1)}\right) F_{L M}(b \bar{b} g)\right\rangle \\
=\left\langleg _ { s } ^ { 2 } ( \frac { \alpha _ { s } } { 4 \pi } ) C _ { F } \left(\mathcal{R}_{\text {int }}^{(1)}-\left[Z_{\alpha_{s}}^{(1)}+Z_{A}^{(1)}+2 \operatorname{Re}\left(\mathbf{Z}_{b \bar{b}}^{(1)}\right)\right]\right.\right. \\
\left.\left.\times\left(\mathcal{S}_{22, \text { int }}^{(0)}-2 \mathcal{S}_{23, \text { int }}^{(0)}+\mathcal{S}_{33, \text { int }}^{(0)}\right)\right) F_{L M}(b \bar{b})\right\rangle .
\end{aligned}
$$

The integrated one-loop soft function $\mathcal{R}_{\text {int }}^{(1)}$ is defined and calculated in section D.3.

\subsection{Double-virtual contribution}

For the $H \rightarrow b \bar{b}$ process, the virtual corrections are described by a single form factor,

$$
\left|\mathcal{M}_{b \bar{b}}\right\rangle=F_{s}\left(M_{H}^{2}, m_{b}^{2}, \mu_{R}^{2}\right)\left|\mathcal{M}_{b \bar{b}}^{(0)}\right\rangle .
$$

In eq. (4.17), $\left|\mathcal{M}_{b \bar{b}}^{(0)}\right\rangle$ is the tree-level $H \rightarrow b \bar{b}$ amplitude, and $F_{s}$ is the scalar heavy-quark form factor, which can be computed perturbatively as

$$
\begin{aligned}
F_{s}\left(M_{H}^{2}, m_{b}^{2}, \mu_{R}^{2}\right)= & 1+\left(\frac{\alpha_{s}\left(\mu_{R}^{2}\right)}{4 \pi}\right) F_{s}^{(1)}\left(M_{H}^{2}, m_{b}^{2}, \mu_{R}^{2}\right) \\
& +\left(\frac{\alpha_{s}\left(\mu_{R}^{2}\right)}{4 \pi}\right)^{2} F_{s}^{(2)}\left(M_{H}^{2}, m_{b}^{2}, \mu_{R}^{2}\right)+\mathcal{O}\left(\alpha_{s}^{3}\right)
\end{aligned}
$$

The expansion coefficients $F_{s}^{(k)}$ depend on Higgs and b-quark masses, the renormalisation scale $\mu_{R}$ and the regulator $\epsilon$. The two-loop heavy-quark form factor was computed in ref. [66] up to $\epsilon^{0}$ terms and to higher orders in $\epsilon$ in refs. [69] and [65]. In our calculation we use the results of ref. [65].

We again use the $\mathbf{Z}$ operator to split the double-virtual correction into divergent terms, that contain all $\epsilon^{-1}$ poles, and a finite remainder. In this case, given the functions $F_{s}^{(1)}\left(M_{H}^{2}, m_{b}^{2}, \mu_{R}^{2}\right)$ and $F_{s}^{(2)}\left(M_{H}^{2}, m_{b}^{2}, \mu_{R}^{2}\right)$ we reconstruct the finite remainders $\left|\mathcal{F}_{b \bar{b}}^{(1)}\right\rangle$ and $\left|\mathcal{F}_{b \bar{b}}^{(2)}\right\rangle$ using the definition in eq. (2.10). We also checked that all $\epsilon^{-1}$ poles of the form factor are in agreement with the $\mathbf{Z}$ operator prediction of eqs. (2.13) and (2.14). The finite double-virtual contribution reads

$$
2 M_{H}\left\langle\mathrm{~d} \Gamma_{\mathrm{VV}}^{\mathrm{F}}(b \bar{b})\right\rangle=\left\langle\left(\frac{\alpha_{s}}{4 \pi}\right)^{2} \mathrm{~d} \Phi_{b \bar{b}}\left[2 \operatorname{Re}\left\langle\mathcal{M}_{b \bar{b}}^{(0)} \mid \mathcal{F}_{b \bar{b}}^{(2)}\right\rangle+\left\langle\mathcal{F}_{b \bar{b}}^{(1)} \mid \mathcal{F}_{b \bar{b}}^{(1)}\right\rangle\right] \mathcal{F}_{\text {kin }}(b \bar{b})\right\rangle .
$$


Furthermore, we distinguish two contributions that contain all $\epsilon^{-1}$ poles. First, we have a term that is proportional to the finite remainder of the one-loop $H \rightarrow b \bar{b}$ matrix element. It is given by

$$
2 M_{H}\left\langle\mathrm{~d} \Gamma_{\mathrm{VV}}^{\mathrm{FR}}\right\rangle=\left\langle\left(\frac{\alpha_{s}}{4 \pi}\right) 2 \operatorname{Re}\left(\mathbf{Z}_{b \bar{b}}^{(1)}\right) F_{L V}^{\mathrm{fin}}(b \bar{b})\right\rangle .
$$

The other contribution containing $\epsilon^{-1}$ poles comes with the Born $H \rightarrow b \bar{b}$ matrix element; it reads

$$
2 M_{H}\left\langle\mathrm{~d} \Gamma_{\mathrm{VV}}^{\mathrm{DU}}\right\rangle=\left\langle\left(\frac{\alpha_{s}}{4 \pi}\right)^{2}\left(2 \operatorname{Re}\left(\mathbf{Z}_{b \bar{b}}^{(2)}\right)+\mathbf{Z}_{b \bar{b}}^{(1)^{\dagger}} \mathbf{Z}_{b \bar{b}}^{(1)}\right) F_{L M}(b \bar{b})\right\rangle .
$$

The expansion coefficients of the $\mathbf{Z}$ operator are given in eqs. (2.13) and (2.14).

\subsection{Pole cancellation}

After collecting formulae for all NNLO contributions in the preceeding subsections, we demonstrate pole cancellation. We begin by considering $\mathrm{d} \Gamma^{\mathrm{FR}}=\mathrm{d} \Gamma_{\mathrm{RV}}^{\mathrm{FR}}+\mathrm{d} \Gamma_{\mathrm{VV}}^{\mathrm{FR}}$. From the real-virtual contribution, eq. (4.15), we obtain the pole term

$$
2 M_{H}\left\langle\mathrm{~d} \Gamma_{\mathrm{RV}}^{\mathrm{FR}}(b \bar{b} g)\right\rangle=\frac{1}{\epsilon}\left[\left(\frac{\alpha_{s}}{4 \pi}\right) 4 C_{F}\left[1+\frac{1+\beta^{2}}{2 \beta} \log \left(\frac{1-\beta}{1+\beta}\right)\right]\left\langle F_{L V}^{\mathrm{fin}}(b \bar{b})\right\rangle\right]+\mathcal{O}\left(\epsilon^{0}\right),
$$

and the explicit expansion of the double-virtual contribution, eq. (4.20), yields

$$
2 M_{H}\left\langle\mathrm{~d} \Gamma_{\mathrm{VV}}^{\mathrm{FR}}(b \bar{b})\right\rangle=-\frac{1}{\epsilon}\left[\left(\frac{\alpha_{s}}{4 \pi}\right) 4 C_{F}\left[1+\frac{1+\beta^{2}}{2 \beta} \log \left(\frac{1-\beta}{1+\beta}\right)\right]\left\langle F_{L V}^{\mathrm{fin}}(b \bar{b})\right\rangle\right],
$$

which obviously cancels in the sum with eq. (4.22).

For the single-unresolved contribution we have $\mathrm{d} \Gamma^{\mathrm{SU}}=\mathrm{d} \Gamma_{\mathrm{RR}}^{\mathrm{SU}}+\mathrm{d} \Gamma_{\mathrm{RV}}^{\mathrm{SU}}$. The double-real contribution, eqs. (4.6) and (4.7), has an expansion which can be cast into the form

$$
\begin{aligned}
2 M_{H}\left\langle\mathrm{~d} \Gamma_{\mathrm{RR}}^{\mathrm{SU}}(b \bar{b} g g+b \bar{b} q \bar{q})\right\rangle \\
=\left\langle( \frac { \alpha _ { s } } { 4 \pi } ) \left[\frac{2 C_{A}}{\epsilon^{2}}+\frac{1}{\epsilon}\left[4 C_{F}+\beta_{0}\left(n_{l}\right)+\frac{C_{A}-2 C_{F}}{v_{23}} \log \left(\frac{1+v_{23}}{1-v_{23}}\right)\right.\right.\right. \\
\left.\left.\left.\quad+2 C_{A} \log \left(\frac{m_{b} \mu_{R}}{2\left(q_{2} \cdot q_{4}\right)}\right)+2 C_{A} \log \left(\frac{m_{b} \mu_{R}}{2\left(q_{3} \cdot q_{4}\right)}\right)\right]\right]\left(I-S_{4}\right) F_{L M}(b \bar{b} g)\right\rangle+\mathcal{O}\left(\epsilon^{0}\right) .
\end{aligned}
$$

A similar expansion holds for the real-virtual single-unresolved contribution, eq. (4.14),

$$
\begin{aligned}
2 M_{H}\left\langle\mathrm{~d} \Gamma_{\mathrm{RV}}^{\mathrm{SU}}(b \bar{b} g)\right\rangle & \\
= & \left\langle( \frac { \alpha _ { s } } { 4 \pi } ) \left[-\frac{2 C_{A}}{\epsilon^{2}}-\frac{1}{\epsilon}\left[4 C_{F}+\beta_{0}\left(n_{l}\right)+\frac{C_{A}-2 C_{F}}{v_{23}} \log \left(\frac{1+v_{23}}{1-v_{23}}\right)\right.\right.\right. \\
& \left.\left.\left.\quad+2 C_{A} \log \left(\frac{m_{b} \mu_{R}}{2\left(q_{2} \cdot q_{4}\right)}\right)+2 C_{A} \log \left(\frac{m_{b} \mu_{R}}{2\left(q_{3} \cdot q_{4}\right)}\right)\right]\right]\left(I-S_{4}\right) F_{L M}(b \bar{b} g)\right\rangle .
\end{aligned}
$$

As before, the poles of the two contributions cancel. 


\begin{tabular}{|lrrrrrrrr|}
\hline & $\mathcal{C}_{C_{F} C_{A}}^{\mathrm{DU},(-3)}$ & \multicolumn{1}{c}{$\mathcal{C}_{C_{F} C_{A}}^{\mathrm{DU},(-2)}$} & $\mathcal{C}_{C_{F}^{2}}^{\mathrm{DU},(-2)}$ & $\mathcal{C}_{C_{F} T_{F} n_{l}}^{\mathrm{DU},(-2)}$ & $\mathcal{C}_{C_{F} C_{A}}^{\mathrm{DU},(-1)}$ & $\mathcal{C}_{C_{F}^{2}}^{\mathrm{DU},(-1)}$ & $\mathcal{C}_{C_{F} T_{F} n_{l}}^{\mathrm{DU},(-1)}$ & $\mathcal{C}_{C_{F} T_{F}}^{\mathrm{DU},(-1)}$ \\
\hline RR & -22.11 & -279.75 & +244.32 & +14.74 & -1777.55 & +2672.58 & +185.77 & 0 \\
RV & +22.11 & +320.28 & -488.64 & -29.47 & +1732.44 & -2672.58 & -161.20 & +257.20 \\
VV & 0 & -40.53 & +244.32 & +14.74 & +45.11 & 0 & -24.56 & -257.20 \\
\hline Sum & $10^{-13}$ & $10^{-10}$ & $10^{-8}$ & $10^{-11}$ & $10^{-6}$ & $10^{-6}$ & $10^{-5}$ & 0 \\
Rel. canc. & $10^{-14}$ & $10^{-13}$ & $10^{-11}$ & $10^{-13}$ & $10^{-10}$ & $10^{-9}$ & $10^{-7}$ & 0 \\
\hline
\end{tabular}

Table 1. Numerical values of the pole coefficients of the double-unresolved term as defined in eq. (4.27). The numerical values correspond to $m_{b}=4.78 \mathrm{GeV}, M_{H}=125.09 \mathrm{GeV}$ and the renormalisation scale is $\mu_{R}=3 M_{H}$. Each column corresponds to a particular colour structure of a given $\epsilon$ pole. The three rows correspond to the double-real, real-virtual, and double-virtual contributions. In the last two rows, we report the absolute and relative level of cancellation after adding up RR $+\mathrm{RV}+\mathrm{VV}$ contributions. The last row is normalised to the largest value of each column.

Finally, we turn to the double-unresolved contribution, given by $\mathrm{d} \Gamma^{\mathrm{DU}}=\mathrm{d} \Gamma_{\mathrm{RR}}^{\mathrm{DU}}+$ $\mathrm{d} \Gamma_{\mathrm{RV}}^{\mathrm{DU}}+\mathrm{d} \Gamma_{\mathrm{VV}}^{\mathrm{DU}}$. The real-virtual and double-virtual contributions are known analytically, but we only have numerical results for the double-real contribution at our disposal. Therefore, we demonstrate pole cancellation numerically. We write the pole terms as

$$
\left\langle\mathrm{d} \Gamma^{\mathrm{DU}}\right\rangle=\left(\frac{\alpha_{s}}{4 \pi}\right)^{2}\left[\frac{\mathcal{C}^{\mathrm{DU},(-3)}}{\epsilon^{3}}+\frac{\mathcal{C}^{\mathrm{DU},(-2)}}{\epsilon^{2}}+\frac{\mathcal{C}^{\mathrm{DU},(-1)}}{\epsilon}\right]\left\langle F_{L M}(b \bar{b})\right\rangle+\mathcal{O}\left(\epsilon^{0}\right),
$$

and further subdivide the pole coefficients according to colour factors,

$$
\mathcal{C}^{\mathrm{DU},(k)}=C_{F} C_{A} \mathcal{C}_{C_{F} C_{A}}^{\mathrm{DU},(k)}+C_{F}^{2} \mathcal{C}_{C_{F}^{2}}^{\mathrm{DU},(k)}+C_{F} T_{F} n_{l} \mathcal{C}_{C_{F} T_{F} n_{l}}^{\mathrm{DU},(k)}+C_{F} T_{F} \mathcal{C}_{C_{F} T_{F}}^{\mathrm{DU},(k)}
$$

We list numerical values for these pole coefficients in table 1 . The pole cancellation reported in the last row occurs to at least 7 to 8 significant digits which proves pole cancellation in the double-unresolved term.

\subsection{Top-quark contribution to the $H \rightarrow b \bar{b}$ decay}

An additional contribution that enters the $H \rightarrow b \bar{b}$ decay process at $\mathcal{O}\left(\alpha_{s}^{2}\right)$ is related to diagrams where the Higgs boson couples to two gluons via a top-quark loop and the final state $b$-quarks are generated by a gluon splitting. The corresponding diagrams are shown in figures $1 \mathrm{a}$ and $1 \mathrm{~b}$ and their contributions to the decay rate are separately finite. This contribution to the total $H \rightarrow b \bar{b}$ width has been computed in refs. [12,13] as an expansion in powers of $x_{t}=\left(M_{H}^{2} / m_{t}^{2}\right)$. An exact result, incorporated into a fully differential calculation, has been recently published in ref. [14]. At the level of the total decay width, the differences between the approximate and the exact results are very small for realistic Higgs and top-quark masses. Since we aim at testing our calculation against results reported in ref. [21], we will use the result of ref. [12] to include the top-quark contribution.

Note that this contribution should naively scale with one power of $y_{b}$ and one power of $y_{t}$, hence we refer to this as the $y_{b} y_{t}$ contribution. However, the amplitudes of figure 1 can interfere with the respective Born amplitudes only if a helicity flip occurs on one of 


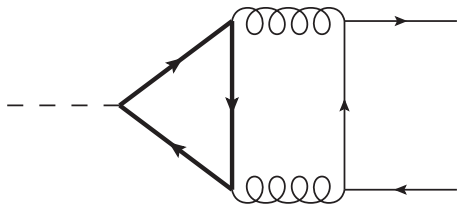

(a) Two-loop $H \rightarrow b \bar{b}$ diagram.

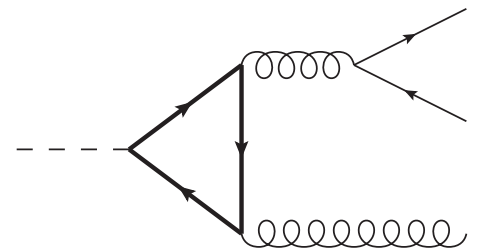

(b) One-loop $H \rightarrow b \bar{b} g$ diagram.

Figure 1. The top-Yukawa contributions to the $H \rightarrow b \bar{b}$ and $H \rightarrow b \bar{b} g$ amplitudes. The solid thick lines represent a top quark while the thin solid lines denote the external $b$-quarks.

the $b$-quark lines. This mechanism provides an additional power of $m_{b}$. Moreover, the topquark loop leads to a suppression factor of $1 / m_{t}$, which makes the overall scaling of the $y_{b} y_{t}$ contribution similar to all other terms, i.e. proportional to a square of the $b$-quark mass.

We write the $y_{b} y_{t}$ contribution to the total decay width as [12]

$$
\Gamma^{y_{b} y_{t}}=\Gamma_{\mathrm{LO}}\left(\frac{\alpha_{s}}{\pi}\right)^{2} \gamma_{2}^{y_{b} y_{t}},
$$

where

$$
\gamma_{2}^{y_{b} y_{t}}=\beta^{-3}\left(f_{2}^{S, 0}+x_{t} f_{2}^{S, 1}+x_{t}^{2} f_{2}^{S, 2}+x_{t}^{3} f_{2}^{S, 3}+\mathcal{O}\left(x_{t}^{4}\right)\right)
$$

and the coefficients $f_{2}^{S, k}$ are given in eq. (2) of ref. [12]. The factor of $\beta^{-3}$ appears since the normalisation factor in eq. (4.28) involves the LO width for the massive $b$-quarks, in contrast to ref. [12].

Since the contribution in eq. (4.28) starts only at $\mathcal{O}\left(\alpha_{s}^{2}\right)$, changes due to decoupling, eq. (A.12), or due to translating the on-shell Yukawa coupling to the $\overline{\mathrm{MS}}$ scheme, eq. (2.33), are of higher order in $\alpha_{s}$ and are discarded.

The real-virtual amplitude related to the diagram in figure $1 \mathrm{~b}$ is computed using the same techniques as the remaining one-loop amplitudes. The double-virtual amplitude of figure 1a is obtained from the result of ref. [12], see eq. (4.28), by subtracting the integrated real-virtual contribution of figure $1 \mathrm{~b}$.

\section{Results}

In this section, we summarise our calculation and present results for the total decay width of the $H \rightarrow b \bar{b}$ decay and a selection of jet rates. We compare these predictions to results already available in the literature. The main goal is to scrutinise our calculation as much as possible to ensure its correctness.

The value of the strong coupling is set to $\alpha_{s}\left(M_{Z}\right)=0.1181$ with $M_{Z}=$ $91.1876 \mathrm{GeV}$ [59]. The evolution is performed at two-loop order with five active flavours using the package RunDec [70, 71]. We use a value for the Higgs boson mass of $M_{H}=125.09 \mathrm{GeV}$ [1]. As a starting point for the $b$-quark mass, we use $\bar{m}_{b}\left(\mu=\bar{m}_{b}\right)=$ $4.18 \mathrm{GeV}$ [59]. From this we calculate the pole mass $m_{b}=4.78 \mathrm{GeV}$, using the two-loop matching formula at a matching scale of $\mu=\bar{m}_{b}\left(\bar{m}_{b}\right)$ implemented in the package RunDec. 
For the value of the Fermi constant we use $G_{F}=1.166378 \times 10^{-5} \mathrm{GeV}^{-2}$ [59]. The central renormalisation scale is taken to be equal to the mass of the Higgs boson, $\mu_{R}=M_{H}$, and for the scale uncertainty estimation we vary it by a factor of $1 / 2$ and 2 .

\subsection{Overview of the calculation}

We combine all contributions discussed in sections 3 and 4 to obtain the full NLO and NNLO results. At leading order in QCD we have

$$
2 M_{H}\left\langle\mathrm{~d} \Gamma_{\mathrm{LO}}^{b \bar{b}}\right\rangle=\left\langle F_{L M}(b \bar{b})\right\rangle .
$$

At NLO we combine real and virtual corrections

$$
\left\langle\mathrm{d} \Gamma_{\delta \mathrm{NLO}}^{b \bar{b}}\right\rangle=\left\langle\mathrm{d} \Gamma_{\mathrm{R}}\right\rangle+\left\langle\mathrm{d} \Gamma_{\mathrm{V}}\right\rangle
$$

which contain finite and unresolved parts as discussed in section 3. The situation is slightly more complicated at NNLO where there are more ingredients entering the final result. The result reads

$$
\left\langle\mathrm{d} \Gamma_{\delta \mathrm{NNLO}}^{b \bar{b}}\right\rangle=\left\langle\mathrm{d} \Gamma_{\mathrm{VV}}\right\rangle+\left\langle\mathrm{d} \Gamma_{\mathrm{RV}}\right\rangle+\left\langle\mathrm{d} \Gamma_{\mathrm{RR}}\right\rangle+\left\langle\mathrm{d} \Gamma_{b \bar{b} b \bar{b}}\right\rangle+\left\langle\mathrm{d} \Gamma_{b \bar{b}}^{y_{b} y_{t}}\right\rangle .
$$

The double-virtual, real-virtual and double-real contributions consist of finite, single- and double-unresolved parts as outlined in section 4.

\subsection{Total width of the $H \rightarrow b \bar{b}$ decay at NNLO}

We start by considering the total width of the decay $H \rightarrow b \bar{b}$; this implies using $\mathcal{F}_{\text {kin }}(b \bar{b} X)=$ 1. We first present the results using the on-shell Yukawa coupling to compare the NNLO decay width to a result in the large Higgs mass limit from ref. [11]. Afterwards, we present the results expressed in terms of the $\overline{\mathrm{MS}}$ Yukawa coupling.

Total width using the on-shell Yukawa coupling. We compare our results for the total decay width, eq. (2.4), to the predictions of ref. [11], which has been obtained as an expansion in $m_{b}^{2} / M_{H}^{2}$. This result was derived for the scenario of a decay of a heavy scalar boson into a pair of top quarks. Nevertheless, it can immediately be translated into the $H \rightarrow b \bar{b}$ decay rate if we neglect the top-loop mediated contribution, discussed in section 4.5. Since the results of ref. [11] are presented in terms of the on-shell Yukawa coupling, we perform the comparison in this scheme.

To scrutinise our results, we split the NLO and NNLO coefficients into independent colour structures

$$
\begin{aligned}
& \gamma_{1}^{b \bar{b}}=C_{F} \gamma_{1}^{C_{F}}, \\
& \gamma_{2}^{b \bar{b}}=C_{F}^{2} \gamma_{2}^{C_{F}^{2}}+C_{F} C_{A} \gamma_{2}^{C_{F} C_{A}}+C_{F} T_{F} n_{l} \gamma_{2}^{C_{F} T_{F} n_{l}}+C_{F} T_{F} \gamma_{2}^{C_{F} T_{F}} .
\end{aligned}
$$

Our findings are summarised in table 2. The first row lists predictions of ref. [11] which are compared to our predictions in the second row. We see a remarkable consistency between the two; the discrepancies are at most at the level of our numerical errors. This good 


\begin{tabular}{|llllll|}
\hline & $\gamma_{1}^{C_{F}}$ & $\gamma_{2}^{C_{F}^{2}}$ & $\gamma_{2}^{C_{F} C_{A}}$ & $\gamma_{2}^{C_{F} T_{F} n_{l}}$ & $\gamma_{2}^{C_{F} T_{F}}$ \\
\hline ref. [11] & -7.446648 & +19.4192 & -53.5558 & +18.6286 & +14.7946 \\
Our res. & $-7.446648(7)$ & $+19.4199(10)$ & $-53.5557(20)$ & $+18.6283(2)$ & $+14.7945(1)$ \\
\hline
\end{tabular}

Table 2. The results for the NLO and NNLO coefficients of the total decay width split into independent colour structures. The renormalisation scale is set to $\mu_{R}=M_{H}$. The uncertainties quoted for our results correspond to errors from numerical integration. We note that the results do not include the $y_{b} y_{t}$ contribution.

agreement is related to the smallness of the expansion parameter in case of a Higgs decay to $b$-quarks, $\left(m_{b}^{2} / M_{H}^{2}\right) \approx 0.00146$.

Note that the numerical values of the NNLO coefficients are much larger than those of the NLO coefficients. Since this is only partially compensated by the additional power of the strong coupling, $\left(\alpha_{s} / \pi\right)$, the NNLO correction in this scheme still amounts to a sizeable change of the total decay width. This behaviour is closely related to large quasi-collinear logarithms, $L=\log \left(m_{b} / M_{H}\right)$, discussed in section 2.5. As already indicated, this issue can be mitigated by reexpressing the results in terms of the $\overline{\mathrm{MS}}$ Yukawa coupling.

Total width using the $\overline{\text { MS }}$ Yukawa coupling. We now express the total decay width in terms of the $\overline{\mathrm{MS}}$ coupling, as defined in eq. (2.34). We use $\bar{m}_{b}\left(\mu=\bar{m}_{b}\right)=4.18 \mathrm{GeV}$ as an input parameter [59] and evolve the $\overline{\mathrm{MS}}$ mass with the RunDec package [70, 71]. We obtain

$$
\bar{m}_{b}\left(\frac{1}{2} M_{H}\right)=2.9814 \mathrm{GeV}, \quad \bar{m}_{b}\left(M_{H}\right)=2.8095 \mathrm{GeV}, \quad \bar{m}_{b}\left(2 M_{H}\right)=2.6641 \mathrm{GeV} .
$$

The evolution is performed with $n_{f}=5$ active flavours at two-loop order. We use these values to evaluate the $\overline{\mathrm{MS}}$ Yukawa coupling. The NLO and NNLO coefficients together with a prediction for a total decay width are presented in table 3 . We first discuss the results without the top-quark contribution, described in section 4.5. For comparison, we also include the NLO and NNLO coefficients obtained analytically in the limit of massless $b$-quarks given in ref. [72].

We see a reasonably good perturbative convergence of the predictions for the total decay width, $\bar{\Gamma}^{b \bar{b}}$. At the central scale, the NNLO corrections change the NLO result by a few percent and the NNLO prediction stays within the NLO scale uncertainties. Since the large quasi-collinear logarithms are removed by switching to the $\overline{\mathrm{MS}}$ Yukawa coupling, the mass corrections are not large. The difference between the NNLO coefficient of the massless and massive predictions is at the level of about $4 \%$ at the central scale, $\mu_{R}=M_{H}$.

Nevertheless, the fully massive treatment of $b$-quarks is desirable because the corrections related to the top-quark Yukawa coupling cannot be incorporated into a fully differential calculation with massless $b$-quarks [18]. Furthermore, it is also important to study the impact of mass effects on the kinematical distributions related to $H \rightarrow b \bar{b}$ decay.

Total decay width including the top-quark contribution. Finally, we incorporate the top-quark contribution into our predictions, according to the discussion presented in 


\begin{tabular}{|llll|}
\hline$\mu_{R}$ & $\frac{1}{2} M_{H}$ & $M_{H}$ & $2 M_{H}$ \\
\hline $\bar{\gamma}_{1}^{b \bar{b}}$ (our res.) & $+3.023597(10)$ & $+5.796203(15)$ & $+8.568783(11)$ \\
$\bar{\gamma}_{1}^{b \bar{b}}$ (Ref. [21]) & +3.024 & +5.798 & +8.569 \\
$\bar{\gamma}_{1}^{b \bar{b}}$ (Ref. [72], $\left.m_{b}=0\right)$ & +2.8941 & +5.6667 & +8.4393 \\
\hline $\bar{\gamma}_{2}^{b \bar{b}}$ (our res., w/o $\left.y_{b} y_{t}\right)$ & $-3.2466(31)$ & $+30.4376(33)$ & $+79.1755(38)$ \\
$\bar{\gamma}_{2}^{b \bar{b}}$ (our res., with $\left.y_{b} y_{t}\right)$ & $+3.7123(31)$ & $+37.3965(33)$ & $+86.1345(38)$ \\
$\bar{\gamma}_{2}^{b \bar{b}}$ (Ref. [21], with $\left.y_{b} y_{t}\right)$ & +3.685 & +37.371 & +86.112 \\
$\bar{\gamma}_{2}^{b \bar{b}}$ (Ref. [72], $\left.m_{b}=0\right)$ & -3.8368 & +29.1467 & +77.1844 \\
\hline $\bar{\Gamma}_{\mathrm{LO}}^{b b}[\mathrm{MeV}]$ & +2.17005 & +1.92702 & +1.73274 \\
$\bar{\Gamma}_{\mathrm{NLO}}^{b \bar{b}}[\mathrm{MeV}]$ & +2.43161 & +2.32781 & +2.21731 \\
$\bar{\Gamma}_{\mathrm{NNLO}}^{b \bar{b}}[\mathrm{MeV}]\left(\right.$ w/o $\left.y_{b} y_{t}\right)$ & $+2.42041(1)$ & $+2.40333(1)$ & $+2.36344(1)$ \\
$\bar{\Gamma}_{\mathrm{NNLO}}^{b \bar{b}}[\mathrm{MeV}]\left(\right.$ with $\left.y_{b} y_{t}\right)$ & $+2.44441(1)$ & $+2.42059(1)$ & $+2.37628(1)$ \\
\hline
\end{tabular}

Table 3. The results for the LO, NLO and NNLO total decay width. The total width is calculated using our results for the expansion coefficients, $\bar{\gamma}_{1}^{b \bar{b}}$ and $\bar{\gamma}_{2}^{b \bar{b}}$. For comparison we include corresponding results from ref. [21]. We also provide results in the limit of massless $b$-quarks from ref. [72], which do not contain the $y_{b} y_{t}$ contribution. The uncertainties quoted for our results correspond to errors from numerical integration.

section 4.5. We set the top-quark pole mass to $m_{t}=173.34 \mathrm{GeV}$ [21]. The NNLO coefficient corresponding to top-quark induced contribution, eq. (4.29), is then $\gamma_{2}^{y_{b} y_{t}}=6.95895$ and is independent of the renormalisation scale. Our predictions are included in table 3.

We see that inclusion of the $y_{b} y_{t}$ contribution increases the total width by about $1.7 \%$ at the central renormalisation scale. A major part of this correction, about $85 \%$, comes from the real-virtual diagrams of the $y_{b} y_{t}$ contribution, see figure $1 \mathrm{~b}$.

Comparing our results for the NLO and NNLO coefficients of the total width to those given in ref. [21], we find excellent agreement at NLO. The NNLO coefficients agree at the level of at least $0.7 \%$ for $\mu_{R}=M_{H} / 2$ and at the sub-permill level for the other scales. Note that for $\bar{\gamma}_{2}^{b \bar{b}}\left(\mu_{R}=M_{H} / 2\right)$ there are large cancellations when converting from the on-shell to the $\overline{\mathrm{MS}}$-scheme Yukawa coupling.

\subsection{Jet rates for $H \rightarrow b \bar{b}$ at NNLO}

We now turn to a discussion of jet rates which provides another stress test of our calculation. We employ the Durham jet algorithm [73] with the default recombination scheme of the parton momenta, $k_{(i j)}=k_{i}+k_{j}$. We use the Fast Jet [74] implementation. We consider two cases of the clustering sequence with $y_{\text {cut }}=0.01$ and $y_{\text {cut }}=0.05$, to facilitate a numerical comparison against ref. [21].

Similar to eq. (2.34), we define an expansion of the differential quantities as

$$
\bar{\Gamma}^{b \bar{b}}(\mathrm{obs})=\bar{\Gamma}_{\mathrm{LO}}^{b \bar{b}}\left[\bar{\gamma}_{0}^{b \bar{b}}(\mathrm{obs})+\left(\frac{\alpha_{s}}{\pi}\right) \bar{\gamma}_{1}^{b \bar{b}}(\mathrm{obs})+\left(\frac{\alpha_{s}}{\pi}\right)^{2} \bar{\gamma}_{2}^{b \bar{b}}(\mathrm{obs})+\mathcal{O}\left(\alpha_{s}^{3}\right)\right]
$$




\begin{tabular}{|lllllll|}
\hline & \multicolumn{3}{c}{$y_{\text {cut }}=0.01$} & \multicolumn{3}{c|}{$y_{\text {cut }}=0.05$} \\
\cline { 2 - 7 }$\mu_{R}$ & $\frac{1}{2} M_{H}$ & $M_{H}$ & $2 M_{H}$ & $\frac{1}{2} M_{H}$ & $M_{H}$ & $2 M_{H}$ \\
\hline $\bar{\gamma}_{1}^{b \bar{b}}$ (2jet) & $-5.0559(1)$ & $-2.2832(1)$ & $+0.4894(1)$ & $+0.2903(1)$ & $+3.0629(1)$ & $+5.8355(1)$ \\
$\bar{\gamma}_{1}^{b \bar{b}}$ (2jet) (Ref. [21]) & -5.055 & -2.282 & +0.490 & +0.291 & +3.063 & +5.836 \\
\hline $\bar{\gamma}_{2}^{b \bar{b}}$ (2jet) (w/o $\left.y_{b} y_{t}\right)$ & $-60.50(3)$ & $-70.68(1)$ & $-65.83(2)$ & $-25.42(1)$ & $-6.59(1)$ & $+27.31(1)$ \\
$\bar{\gamma}_{2}^{b \bar{b}}$ (2jet) (with $\left.y_{b} y_{t}\right)$ & $-56.40(3)$ & $-66.58(1)$ & $-61.73(2)$ & $-19.52(1)$ & $-0.69(1)$ & $+33.21(1)$ \\
$\bar{\gamma}_{2}^{b \bar{b}}$ (2jet) (Ref. [21]) & -56.351 & -66.532 & -61.658 & -19.496 & -0.650 & +33.250 \\
\hline $\bar{\gamma}_{1}^{b \bar{b}}$ (3jet) & $+8.0794(1)$ & $+8.0794(1)$ & $+8.0794(1)$ & $+2.7333(1)$ & $+2.7333(1)$ & $+2.7333(1)$ \\
$\bar{\gamma}_{1}^{b \bar{b}}$ (3jet) (Ref. [21]) & +8.079 & +8.079 & +8.079 & +2.733 & +2.733 & +2.733 \\
\hline $\bar{\gamma}_{2}^{b \bar{b}}$ (3jet) (w/o $\left.y_{b} y_{t}\right)$ & $+34.09(3)$ & $+77.96(1)$ & $+121.84(2)$ & $+21.25(1)$ & $+36.09(1)$ & $+50.94(1)$ \\
$\bar{\gamma}_{2}^{b \bar{b}}$ (3jet) (with $\left.y_{b} y_{t}\right)$ & $+36.95(3)$ & $+80.82(1)$ & $+124.70(2)$ & $+22.30(1)$ & $+37.15(1)$ & $+51.99(1)$ \\
$\bar{\gamma}_{2}^{b \bar{b}}$ (3jet) (Ref. [21]) & +36.873 & +80.741 & +124.609 & +22.256 & +37.096 & +51.937 \\
\hline $\bar{\gamma}_{2}^{b \bar{b}}$ (4jet) & $+23.164(1)$ & $+23.163(1)$ & $+23.163(1)$ & $+0.9323(1)$ & $+0.9322(1)$ & $+0.9322(1)$ \\
$\bar{\gamma}_{2}^{b \bar{b}}$ (4jet) (Ref. [21]) & +23.163 & +23.163 & +23.163 & +0.926 & +0.926 & +0.926 \\
\hline
\end{tabular}

Table 4. The jet rates expansion coefficients $\bar{\gamma}_{i}^{b \bar{b}}$ (obs) as defined in eq. (5.7) and computed using the Durham clustering algorithm with $y_{\text {cut }}=0.01$ and $y_{\text {cut }}=0.05$ for various choices of the renormalisation scale. Whenever necessary, we report results without and with top-quark contributions.

where "obs" denotes a generic observable and the $\overline{\mathrm{MS}}$ quantities are related to their counterparts in the pole scheme via

$$
\begin{aligned}
& \bar{\gamma}_{0}^{b \bar{b}}(\mathrm{obs})=\gamma_{0}^{b \bar{b}}(\mathrm{obs}) \\
& \bar{\gamma}_{1}^{b \bar{b}}(\mathrm{obs})=\gamma_{1}^{b \bar{b}}(\mathrm{obs})+r_{1} \gamma_{0}^{b \bar{b}}(\mathrm{obs}), \\
& \bar{\gamma}_{2}^{b \bar{b}}(\mathrm{obs})=\gamma_{2}^{b \bar{b}}(\mathrm{obs})+r_{1} \gamma_{1}^{b \bar{b}}(\mathrm{obs})+r_{2} \gamma_{0}^{b \bar{b}}(\mathrm{obs}) .
\end{aligned}
$$

Note that the sum of expansion coefficients of all relevant jet multiplicities, $\bar{\gamma}_{i}^{b \bar{b}}(n$ jet $)$, yields the expansion coefficient of the total decay width $\bar{\gamma}_{i}^{b \bar{b}}$.

We keep using the setup presented in section 5.2 and report results for the two-, threeand four-jet rates in table 4 . We present these results with and without top-quark contributions whenever relevant, i.e. for the two- and three-jet rate NNLO coefficients. We see that the NLO coefficient of the three-jet rate and the NNLO coefficient of the four-jet rate are scale-independent since they involve only tree-level subprocesses. We also observe a migration of events from higher to lower jet multiplicities when jet-cut parameter is increased.

For the NLO and NNLO coefficients of the jet rates we see agreement between our result and that of ref. [21] at the level of $0.1 \%$ to $0.2 \%$ for all quantities considered for $y_{\text {cut }}=0.01$. Similar agreement is observed for $y_{\text {cut }}=0.05$ with the exception of $\bar{\gamma}_{2}^{b \bar{b}}\left(2\right.$ jet, $\left.\mu_{R}=M_{H}\right)$, which however suffers from large cancellations in the course of the on-shell to $\overline{\mathrm{MS}}$-scheme conversion, and the four-jet rates.

Finally, in table 5 we present the total jet rates for the central choice of the renormalisation scale, $\mu_{R}=M_{H}$. We see a clear hierarchy of the jet rates, with the two-jet rate being the largest and the four-jet rate the smallest for both clustering parameters. This is more pronounced for the larger jet cut, $y_{\text {cut }}=0.05$, as expected. 


\begin{tabular}{|lll|}
\hline$y_{\text {cut }}$ & 0.01 & 0.05 \\
\hline $\bar{\Gamma}^{b \bar{b}}(2$ jet $)[\mathrm{MeV}]$ & $1.60395(4)$ & $2.13710(2)$ \\
$\bar{\Gamma}^{b \bar{b}}(3$ jet $)[\mathrm{MeV}]$ & $0.75917(4)$ & $0.28118(2)$ \\
$\bar{\Gamma}^{b \bar{b}}(4$ jet $)[\mathrm{MeV}]$ & 0.05747 & 0.0023 \\
\hline
\end{tabular}

Table 5. The total jet rates at NNLO for $\mu_{R}=M_{H}$ using the Durham clustering algorithm with $y_{\text {cut }}=0.01$ and $y_{\text {cut }}=0.05$, including the $y_{b} y_{t}$ contribution.

To conclude, we note that the main objective of this section was to put our NNLO calculation under a thorough examination. We performed a series of comparisons of our calculation against various results available in the literature. The positive outcome of these checks assures us of the validity of our approach.

\section{Conclusions}

In this paper, we presented an independent calculation of the NNLO QCD corrections to the Higgs boson decay into massive $b$-quarks. We worked in the framework of the nested soft-collinear subtraction scheme introduced in refs. [18, 26-28].

A complete discussion of all necessary NNLO contributions was presented in section 4 . In particular, we demonstrated cancellation of all $\epsilon^{-1}$ poles, related to soft and collinear singularities of QCD amplitudes. The cancellation was obtained pointwise in phase space, without referring to a specific form of the matrix elements. Furthermore, a full treatment of the $b$-quark mass allowed for an inclusion of the additional contribution which originates from a direct interaction of top quarks with the Higgs boson, at a differential level.

Our fully differential calculation was implemented in a computer program. We carefully tested it by performing a number of cross-checks with results available in the literature both for the total decay width $[11,21]$ and jet rates [21].

We note that the calculation presented in this paper is an important step towards a broader phenomenological goal, namely, combining a description of Higgs boson production with its decay into massive $b$-quarks. In the context of associated Higgs production, where large radiative corrections for important observables have been reported [18, 19, 22], a thorough study of $b$-quark mass effects is an interesting topic for future research.

\section{Acknowledgments}

We wish to thank Kirill Melnikov for stimulating discussions on the topics treated here and very valuable comments on the manuscript. We would like to thank Peter Marquard and Matthias Steinhauser for useful conversations about renormalisation and decoupling. We are also grateful to the authors of ref. [21] for useful correspondence regarding the numerical cross-checks. This research was supported by the Deutsche Forschungsgemeinschaft (DFG, German Research Foundation) under grant 396021762 - TRR 257. The work of A.B. was in part supported by BMBF grant 05H18VKCC1. 


\section{A Renormalisation}

The subtraction scheme that we apply in this calculation is formulated in terms of UVrenormalised amplitudes. Here, we describe the renormalisation prescriptions used in our calculation.

We employ a hybrid scheme in which we renormalise the quark and gluon fields, the quark masses and the Yukawa coupling in an on-shell scheme, whereas we use the $\overline{\mathrm{MS}}$ scheme with five active flavours for the strong coupling constant. We write

$$
\alpha_{s, b}=\left(\mu_{R}^{2}\right)^{\epsilon} S_{\epsilon} Z_{\alpha_{s}} \alpha_{s}, \quad \psi_{b}^{0}=\sqrt{Z_{\psi}} \psi_{b}, \quad G_{\mu}^{0, a}=\sqrt{Z_{A}} G_{\mu}^{a}, \quad m_{b}^{0}=Z_{m} m_{b}, \quad y_{b}^{0}=Z_{m} y_{b},
$$

where $S_{\epsilon}=(4 \pi)^{-\epsilon} \exp \left(\epsilon \gamma_{\mathrm{E}}\right) .{ }^{4}$ Due to the choice to enforce the relation $y_{b}=m_{b}\left(2 \sqrt{2} G_{F}\right)^{1 / 2}$, through the renormalisation condition of the Yukawa coupling, the mass and Yukawa coupling share the same renormalisation constant. The remaining renormalisation constants (for the massless quarks, ghosts and the gauge parameter) do not explicitly appear in our calculation. The renormalisation of the strong coupling constant requires $[75,76]$

$$
Z_{\alpha_{s}}=1+\frac{\alpha_{s}}{4 \pi} Z_{\alpha_{s}}^{(1)}+\mathcal{O}\left(\alpha_{s}^{2}\right)=1-\frac{\alpha_{s}}{4 \pi} \frac{\beta_{0}\left(n_{l}+1\right)}{\epsilon}+\mathcal{O}\left(\alpha_{s}^{2}\right)
$$

with $\beta_{0}\left(n_{l}+1\right)=\frac{11}{3} C_{A}-\frac{4}{3} T_{F}\left(n_{l}+1\right)$. The mass and field renormalisation constants for the massive quark, $Z_{\psi}$ and $Z_{m}$, are given by [77]

$$
\begin{aligned}
Z_{m}= & 1+\frac{\alpha_{s, b}}{4 \pi} Z_{m}^{(1)}+\left(\frac{\alpha_{s, b}}{4 \pi}\right)^{2} Z_{m}^{(2)}+\mathcal{O}\left(\alpha_{s, b}^{3}\right) \\
= & 1+\frac{\alpha_{s, b}}{4 \pi}\left(m^{2}\right)^{-\epsilon} S_{\epsilon}^{-1} C_{F}\left(-\frac{3}{\epsilon}-4-\epsilon\left(8+\frac{3}{2} \zeta_{2}\right)\right) \\
& +\left(\frac{\alpha_{s, b}}{4 \pi}\right)^{2}\left(m^{2}\right)^{-2 \epsilon} S_{\epsilon}^{-2}\left[C_{F}^{2}\left(\frac{9}{2 \epsilon^{2}}+\frac{45}{4 \epsilon}+\frac{199}{8}+\left(-\frac{51}{2}+48 \ln (2)\right) \zeta_{2}-12 \zeta_{3}\right)\right. \\
& +C_{A} C_{F}\left(-\frac{11}{2 \epsilon^{2}}-\frac{91}{4 \epsilon}-\frac{605}{8}+\left(\frac{5}{2}-24 \ln (2)\right) \zeta_{2}+6 \zeta_{3}\right) \\
& \left.+C_{F} T_{F}\left(\frac{2}{\epsilon^{2}}+\frac{7}{\epsilon}+\frac{69}{2}-14 \zeta_{2}\right)+C_{F} T_{F} n_{l}\left(\frac{2}{\epsilon^{2}}+\frac{7}{\epsilon}+\frac{45}{2}+10 \zeta_{2}\right)\right]+\mathcal{O}\left(\alpha_{s, b}^{3}\right), \\
Z_{\psi}= & 1+\frac{\alpha_{s, b}}{4 \pi} Z_{\psi}^{(1)}+\left(\frac{\alpha_{s, b}}{4 \pi}\right)^{2} Z_{\psi}^{(2)}+\mathcal{O}\left(\alpha_{s, b}^{3}\right) \\
= & 1+\frac{\alpha_{s, b}}{4 \pi}\left(m^{2}\right)^{-\epsilon} S_{\epsilon}^{-1} C_{F}\left(-\frac{3}{\epsilon}-4-\epsilon\left(8+\frac{3}{2} \zeta_{2}\right)\right) \\
& +\left(\frac{\alpha_{s, b}}{4 \pi}\right)^{2}\left(m^{2}\right)^{-2 \epsilon} S_{\epsilon}^{-2}\left[C_{F}^{2}\left(\frac{9}{2 \epsilon^{2}}+\frac{51}{4 \epsilon}+\frac{433}{8}+\left(-\frac{147}{2}+96 \ln (2)\right) \zeta_{2}-24 \zeta_{3}\right)\right. \\
& +C_{A} C_{F}\left(-\frac{11}{2 \epsilon^{2}}-\frac{101}{4 \epsilon}-\frac{803}{8}+\left(\frac{49}{2}-48 \ln (2)\right) \zeta_{2}+12 \zeta_{3}\right) \\
& \left.+C_{F} T_{F}\left(\frac{4}{\epsilon^{2}}+\frac{19}{3 \epsilon}+\frac{1139}{18}-28 \zeta_{2}\right)+C_{F} T_{F} n_{l}\left(\frac{2}{\epsilon^{2}}+\frac{9}{\epsilon}+\frac{59}{2}+10 \zeta_{2}\right)\right]+\mathcal{O}\left(\alpha_{s, b}^{3}\right) .
\end{aligned}
$$

\footnotetext{
${ }^{4}$ This agrees with the renormalisation scheme in [65], but differs from [66] where a different choice for the renormalisation of the strong coupling is used. Both schemes yield the same result for physical observables, but differ by terms proportional to $\zeta_{2}$ in IR-divergent intermediate steps; this difference has already been discussed in ref. [21].
} 
The gluon field renormalisation constant is non-vanishing in the on-shell scheme due to heavy-quark loops and reads [78, 79]

$$
Z_{A}=1+\frac{\alpha_{s, b}}{4 \pi} Z_{A}^{(1)}+\mathcal{O}\left(\alpha_{s, b}^{2}\right)=1+\frac{\alpha_{s, b}}{4 \pi} \frac{\beta_{0, Q}}{\epsilon}\left(\frac{\mu_{R}^{2}}{m_{b}^{2}}\right)^{\epsilon} \Gamma(1+\epsilon) e^{\epsilon \gamma_{\mathrm{E}}}+\mathcal{O}\left(\alpha_{s, b}^{2}\right)
$$

with $\beta_{0, Q}=-\frac{4}{3} T_{F}$.

For single-virtual and double-virtual amplitudes of the $H \rightarrow b \bar{b}$ process we start with the unrenormalised amplitude calculated from all one-particle-irreducible Feynman diagrams. Expanded in terms of the bare strong coupling, we have

$$
\left|\hat{\mathcal{M}}_{b \bar{b}}\right\rangle=\left|\hat{\mathcal{M}}_{b \bar{b}}^{(0)}\right\rangle+\frac{\alpha_{s, b}}{4 \pi}\left|\hat{\mathcal{M}}_{b \bar{b}}^{(1)}\right\rangle+\left(\frac{\alpha_{s, b}}{4 \pi}\right)^{2}\left|\hat{\mathcal{M}}_{b \bar{b}}^{(2)}\right\rangle+\mathcal{O}\left(\alpha_{s, b}^{3}\right)
$$

and together with the LSZ factors we obtain the renormalised amplitude as

$$
\begin{aligned}
\left|\mathcal{M}_{b \bar{b}}\right\rangle= & Z_{m} Z_{\psi}\left|\hat{\mathcal{M}}_{b \bar{b}}\right\rangle+\left(\frac{\alpha_{s, b}}{4 \pi}\right)^{2} Z_{m}^{(1)}\left|\hat{\mathcal{C}}_{m, b \bar{b}}^{(1)}\right\rangle+\mathcal{O}\left(\alpha_{s, b}^{3}\right) \\
= & \left|\hat{\mathcal{M}}_{b \bar{b}}^{(0)}\right\rangle+\frac{\alpha_{s}}{4 \pi}\left(\mu_{R}^{2}\right)^{\epsilon} S_{\epsilon}\left[\left|\hat{\mathcal{M}}_{b \bar{b}}^{(1)}\right\rangle+\left|\hat{\mathcal{M}}_{b \bar{b}}^{(0)}\right\rangle\left(Z_{m}^{(1)}+Z_{\psi}^{(1)}\right)\right] \\
& +\left(\frac{\alpha_{s}}{4 \pi}\right)^{2}\left[\left(\mu_{R}^{2}\right)^{\epsilon} S_{\epsilon} Z_{\alpha_{s}}^{(1)}\left(\left|\hat{\mathcal{M}}_{b \bar{b}}^{(1)}\right\rangle+\left|\hat{\mathcal{M}}_{b \bar{b}}^{(0)}\right\rangle\left(Z_{m}^{(1)}+Z_{\psi}^{(1)}\right)\right)\right. \\
& +\left(\mu_{R}^{2}\right)^{2 \epsilon} S_{\epsilon}^{2}\left(\left|\hat{\mathcal{M}}_{b \bar{b}}^{(2)}\right\rangle+\left(Z_{m}^{(1)}+Z_{\psi}^{(1)}\right)\left|\hat{\mathcal{M}}_{b \bar{b}}^{(1)}\right\rangle\right. \\
& \left.\left.+\left(Z_{m}^{(2)}+Z_{m}^{(1)} Z_{\psi}^{(1)}+Z_{\psi}^{(2)}\right)\left|\hat{\mathcal{M}}_{b \bar{b}}^{(0)}\right\rangle+Z_{m}^{(1)}\left|\hat{\mathcal{C}}_{m, b \bar{b}}^{(1)}\right\rangle\right)\right]+\mathcal{O}\left(\alpha_{s}^{3}\right) \\
= & \left|\mathcal{M}_{b \bar{b}}^{(0)}\right\rangle+\frac{\alpha_{s}}{4 \pi}\left|\mathcal{M}_{b \bar{b}}^{(1)}\right\rangle+\left(\frac{\alpha_{s}}{4 \pi}\right)^{2}\left|\mathcal{M}_{b \bar{b}}^{(2)}\right\rangle+\mathcal{O}\left(\alpha_{s}^{3}\right)
\end{aligned}
$$

Here, $\left|\hat{\mathcal{C}}_{m, b \bar{b}}^{(1)}\right\rangle$ denotes the amplitude of the mass counterterm diagrams and $\left|\mathcal{M}_{b \bar{b}}^{(k)}\right\rangle$ denotes the renormalised amplitude of order $\alpha_{s}^{k}$.

In contrast to the Born process, the real-emission amplitude for $H \rightarrow b \bar{b} g$ starts at $\mathcal{O}\left(\alpha_{s}\right)$, which means that coupling renormalisation already affects the one-loop correction. In accordance with eq. (2.9) we leave powers of the strong coupling constant related to real emissions implicit in the amplitude and write the perturbative expansion of the bare $H \rightarrow b \bar{b} g$ amplitude as

$$
\left|\hat{\mathcal{M}}_{b \bar{b} g}\right\rangle=\left|\hat{\mathcal{M}}_{b \bar{b} g}^{(0)}\right\rangle+\frac{\alpha_{s, b}}{4 \pi}\left|\hat{\mathcal{M}}_{b \bar{b} g}^{(1)}\right\rangle+\mathcal{O}\left(\alpha_{s, b}^{3}\right)
$$

In analogy to eq. (A.7), the $H \rightarrow b \bar{b} g$ process is renormalised via

$$
\begin{aligned}
\left|\mathcal{M}_{b \bar{b} g}\right\rangle= & Z_{m} Z_{\psi} \sqrt{Z_{A}}\left|\hat{\mathcal{M}}_{b \bar{b} g}\right\rangle+\frac{\alpha_{s, b}}{4 \pi} Z_{m}^{(1)}\left|\hat{\mathcal{C}}_{m, b \bar{b} g}^{(1)}\right\rangle+\mathcal{O}\left(\alpha_{s, b}^{3}\right) \\
= & \left(\mu_{R}^{2}\right)^{\epsilon} S_{\epsilon}\left|\hat{\mathcal{M}}_{b \bar{b} g}^{(0)}\right\rangle+\frac{\alpha_{s}}{4 \pi}\left[\left(\mu_{R}^{2}\right)^{\epsilon} S_{\epsilon} Z_{\alpha_{s}}^{(1)}\left|\hat{\mathcal{M}}_{b \bar{b} g}^{(0)}\right\rangle\right. \\
& \left.+\left(\mu_{R}^{2}\right)^{2 \epsilon} S_{\epsilon}^{2}\left(\left|\hat{\mathcal{M}}_{b \bar{b} g}^{(1)}\right\rangle+\left(\frac{Z_{A}^{(1)}}{2}+Z_{m}^{(1)}+Z_{\psi}^{(1)}\right)\left|\hat{\mathcal{M}}_{b \bar{b} g}^{(0)}\right\rangle+Z_{m}^{(1)}\left|\hat{\mathcal{C}}_{m, b \bar{b} g}^{(1)}\right\rangle\right)\right]+\mathcal{O}\left(\alpha_{s}^{3}\right) \\
= & \left(\mu_{R}^{2}\right)^{\epsilon} S_{\epsilon}\left(\left|\mathcal{M}_{b \bar{b} g}^{(0)}\right\rangle+\frac{\alpha_{s}}{4 \pi}\left|\mathcal{M}_{b \bar{b} g}^{(1)}\right\rangle\right)+\mathcal{O}\left(\alpha_{s}^{3}\right) .
\end{aligned}
$$


Note that for the expansion of the renormalised amplitude, we pull out a global factor of $\left(\mu_{R}^{2}\right)^{\epsilon} S_{\epsilon}$ and we move it to the normalisation of the phase-space measure, see eq. (2.16). Analogously, the renormalised double-real amplitudes are written as

$$
\begin{aligned}
& \left|\mathcal{M}_{b \bar{b} g g}\right\rangle=\left(\mu_{R}^{2}\right)^{2 \epsilon} S_{\epsilon}^{2}\left|\mathcal{M}_{b \bar{b} g g}^{(0)}\right\rangle+\mathcal{O}\left(\alpha_{s}^{3}\right), \\
& \left|\mathcal{M}_{b \bar{b} q \bar{q}}\right\rangle=\left(\mu_{R}^{2}\right)^{2 \epsilon} S_{\epsilon}^{2}\left|\mathcal{M}_{b \bar{b} q \bar{q}}^{(0)}\right\rangle+\mathcal{O}\left(\alpha_{s}^{3}\right) .
\end{aligned}
$$

Both for the calculation of the amplitudes and the application of the subtraction scheme, we choose to work in a theory with $n_{f}=n_{l}+1$ active flavours. This amounts to renormalising the strong coupling with $\beta_{0}\left(n_{l}+1\right)$ and the gluon field with a non-trivial renormalisation constant $\left(\sqrt{Z_{A}} \neq 1\right)$. This procedure takes care of insertions of heavyquark loops on gluon into propagators and external gluon legs.

A possible alternative [31] is to formulate the subtraction scheme in a theory where the strong coupling evolves with $n_{l}$ active flavours. If we calculate the amplitudes in the renormalisation scheme with $n_{l}+1$ active flavours described above, we can use the decoupling relation $[80-86]$

$$
\alpha_{s}^{\left(n_{l}\right)}=\zeta_{\alpha_{s}} \alpha_{s}^{\left(n_{l}+1\right)}
$$

to absorb the effects of the heavy-quark loops into the running of $\alpha_{s}$. We stress that in this case, the higher-order terms in $\epsilon$ of the decoupling constant $\zeta_{\alpha_{s}}$ need to be taken into account. To $\mathcal{O}\left(\alpha_{s}\right)$ we need [87]

$$
\zeta_{\alpha_{s}}=1+\frac{\alpha_{s}^{\left(n_{l}\right)}}{4 \pi} \frac{\beta_{0, Q}}{\epsilon}\left[\left(\frac{\mu_{R}^{2}}{m_{b}^{2}}\right)^{\epsilon} \Gamma(1+\epsilon) e^{\epsilon \gamma_{\mathrm{E}}}-1\right]+\mathcal{O}\left(\left(\alpha_{s}^{\left(n_{l}\right)}\right)^{2}\right) .
$$

The subtraction scheme then operates on amplitudes in a theory with $n_{l}$ active flavours. Finally, after all IR poles cancel we can use eq. (A.12) in the opposite direction to go back to the theory with $n_{l}+1$ active flavours. The results of this procedure are identical to the results obtained when working with $n_{l}+1$ active flavours throughout the whole calculation.

\section{B Useful formulae}

For the convenience of the reader, in this appendix, we collect useful formulae that are available in the literature and are used in our calculation.

\section{B.1 Anomalous dimensions for IR factorisation}

Below, we give explicit expressions for the anomalous dimensions that appear in the $\mathbf{Z}$ operator. All of these coefficients were taken from ref. [31]; they can further be traced to refs. [46, 51].

We write an expansion of the anomalous dimensions in terms of the strong coupling constant,

$$
\gamma_{i}\left(\alpha_{s}\right)=\sum_{n=0}^{\infty} \gamma_{i}^{(n)}\left(\frac{\alpha_{s}}{4 \pi}\right)^{n+1}
$$

where $i$ stands for the type of the anomalous dimension. We report formulae for the massless and massive cusp anomalous dimensions, heavy-quark and gluon anomalous dimensions. 
The expansion coefficients of the massless cusp anomalous dimension are given by

$$
\gamma_{\text {cusp }}^{(0)}=4, \quad \gamma_{\text {cusp }}^{(1)}=\left(\frac{268}{9}-\frac{4}{3} \pi^{2}\right) C_{A}-\frac{80}{9} T_{F} n_{l},
$$

with $n_{l}$ being a number of massless quark flavours. For the cusp anomalous dimension of massive emitters the expansion coefficients depend on

$$
v_{i j}=\sqrt{1-\frac{m_{i}^{2} m_{j}^{2}}{\left(q_{i} \cdot q_{j}\right)^{2}}}
$$

and read

$$
\begin{aligned}
\gamma_{\text {cusp }, Q}^{(0)}(v)= & \gamma_{\text {cusp }}^{(0)} \frac{1}{v}\left[\frac{1}{2} \ln \left(\frac{1+v}{1-v}\right)-i \pi\right] \\
\gamma_{\text {cusp }, Q}^{(1)}(v)= & \gamma_{\text {cusp }}^{(1)} \frac{1}{v}\left[\frac{1}{2} \ln \left(\frac{1+v}{1-v}\right)-i \pi\right]+8 C_{A}\left\{\zeta_{3}-\frac{5}{6} \pi^{2}+\frac{1}{4} \ln ^{2}\left(\frac{1+v}{1-v}\right)\right. \\
& +\frac{1}{v^{2}}\left[\frac{1}{24} \ln ^{3}\left(\frac{1+v}{1-v}\right)+\ln \left(\frac{1+v}{1-v}\right)\left(\frac{1}{2} \operatorname{Li}_{2}\left(\frac{1-v}{1+v}\right)-\frac{5 \pi^{2}}{12}\right)\right. \\
& \left.+\operatorname{Li}_{3}\left(\frac{1-v}{1+v}\right)-\zeta_{3}\right]+\frac{1}{v}\left[\frac{5}{6} \pi^{2}+\frac{5}{12} \pi^{2} \ln \left(\frac{1+v}{1-v}\right)-\frac{1}{4} \ln ^{2}\left(\frac{1+v}{1-v}\right)\right. \\
& \left.-\ln \left(\frac{2 v}{1+v}\right) \ln \left(\frac{1+v}{1-v}\right)-\frac{1}{24} \ln ^{3}\left(\frac{1+v}{1-v}\right)+\operatorname{Li}_{2}\left(\frac{1-v}{1+v}\right)\right] \\
& +i \pi\left\{\frac{1}{v^{2}}\left[\frac{\pi^{2}}{6}-\frac{1}{4} \ln ^{2}\left(\frac{1+v}{1-v}\right)-\operatorname{Li}_{2}\left(\frac{1-v}{1+v}\right)\right]\right. \\
& +\frac{1}{v}\left[-\frac{\pi^{2}}{6}+2 \ln \left(\frac{2 v}{1+v}\right)+\ln \left(\frac{1+v}{1-v}\right)+\frac{1}{4} \ln ^{2}\left(\frac{1+v}{1-v}\right)\right] \\
& \left.\left.-\ln \left(\frac{1+v}{1-v}\right)\right\}\right\}
\end{aligned}
$$

Furthermore, the heavy-quark anomalous dimensions are

$$
\gamma_{Q}^{(0)}=-2 C_{F}, \quad \gamma_{Q}^{(1)}=C_{F} C_{A}\left(\frac{2}{3} \pi^{2}-\frac{98}{9}-4 \zeta_{3}\right)+\frac{40}{9} C_{F} T_{F} n_{l},
$$

while for gluons we have

$$
\gamma_{g}^{(0)}=-\beta_{0}\left(n_{l}\right)=-\frac{11}{3} C_{A}+\frac{4}{3} T_{F} n_{l}
$$

\section{B.2 Coefficients for on-shell to $\overline{\mathrm{MS}}$-scheme conversion}

The coefficients for the conversion relation between the on-shell and $\overline{\mathrm{MS}}$ Yukawa coupling, defined in eq. (2.33), are given by $[21,88]$

$$
r_{1}=-2 d_{1}, \quad r_{2}=3 d_{1}^{2}-2 d_{2},
$$


and

$$
\begin{aligned}
d_{1}\left(m_{b}, \mu\right)= & -C_{F}\left(1+\frac{3}{4} L\right) \\
d_{2}\left(m_{b}, \mu\right)= & C_{F}^{2}\left[\frac{7}{128}-\frac{3}{4} \zeta_{3}+3 \ln (2) \zeta_{2}-\frac{15}{8} \zeta_{2}+\frac{21}{32} L+\frac{9}{32} L^{2}\right] \\
& +C_{A} C_{F}\left[-\frac{1111}{384}+\frac{3}{8} \zeta_{3}+\frac{1}{2} \zeta_{2}-\frac{3}{2} \ln (2) \zeta_{2}-\frac{185}{96} L-\frac{11}{32} L^{2}\right] \\
& +C_{F} T_{F} n_{l}\left[\frac{71}{96}+\frac{1}{2} \zeta_{2}+\frac{13}{24} L+\frac{1}{8} L^{2}\right]+C_{F} T_{F}\left[\frac{143}{96}-\zeta_{2}+\frac{13}{24} L+\frac{1}{8} L^{2}\right]
\end{aligned}
$$

with the abbreviation $L=\ln \left(\mu^{2} / m_{b}^{2}\right)$.

\section{Factorisation formulae}

Here, we collect the factorisation formulae which are necessary to evaluate the singular limits of the squared matrix elements. We specialise to the $H \rightarrow b \bar{b} g, H \rightarrow b \bar{b} g g$ and $H \rightarrow b \bar{b} q \bar{q}$ processes. For a useful collection of factorisation formulae for general NNLO QCD processes, we refer the reader to ref. [31].

\section{C.1 Single-collinear factorisation}

In order to discuss the single-collinear limit of the $H \rightarrow b \bar{b} g g$ and $H \rightarrow b \bar{b} q \bar{q}$ matrix element, we have to define a perpendicular direction $k_{\perp}^{\mu}$ which determines how the collinear limit is approached. We choose

$$
k_{\perp}^{\mu}=\lim _{\eta \rightarrow 0} \frac{\hat{q}_{5}-\hat{q}_{4}}{\left\|\hat{q}_{5}-\hat{q}_{4}\right\|},
$$

where $\eta$ refers to the phase-space parametrisation in eq. (2.31). Then the factorisation formula in the single-collinear limit reads

$$
C_{45}\left|\mathcal{M}_{b \bar{b} i j}^{(0)}\right|^{2}=\frac{g_{s}^{2}}{\left(q_{4} \cdot q_{5}\right)}\left\langle\mathcal{M}_{b \bar{b} g, \mu}^{(0)}\left|\mathcal{P}_{i j}^{(0), \mu \nu}\left(z, k_{\perp} ; \epsilon\right)\right| \mathcal{M}_{b \bar{b} g, \nu}^{(0)}\right\rangle
$$

where $i j$ stands for either the $g g$ or $q \bar{q}$ channel and $\left|\mathcal{M}_{b \bar{b} g, \nu}^{(0)}\right\rangle$ is the spin-correlated $H \rightarrow b \bar{b} g$ amplitude with the gluon polarisation vector removed, i.e.

$$
\left|\mathcal{M}_{b \bar{b} g}^{(0)}\right\rangle=\left(\varepsilon^{\mu}\left(q_{4}, \lambda\right)\right)^{*}\left|\mathcal{M}_{b \bar{b} g, \mu}^{(0)}\right\rangle .
$$

The splitting functions $\mathcal{P}_{i j}^{(0), \mu \nu}$ carry Lorentz indices which are contracted with the corresponding index of the gluon. For the $g g$ and $q \bar{q}$ channels they read

$$
\begin{aligned}
& \mathcal{P}_{g g}^{(0), \mu \nu}\left(z, k_{\perp} ; \epsilon\right)=2 C_{A}\left[-g^{\mu \nu}\left(\frac{z}{1-z}+\frac{1-z}{z}\right)-2(1-\epsilon) z(1-z) \frac{k_{\perp}^{\mu} k_{\perp}^{\nu}}{k_{\perp}^{2}}\right], \\
& \mathcal{P}_{q \bar{q}}^{(0), \mu \nu}\left(z, k_{\perp} ; \epsilon\right)=T_{F}\left[-g^{\mu \nu}+4 z(1-z) \frac{k_{\perp}^{\mu} k_{\perp}^{\nu}}{k_{\perp}^{2}}\right],
\end{aligned}
$$


with

$$
z=\frac{E_{4}}{E_{4}+E_{5}}=1-\frac{\xi_{2}}{2} .
$$

As the factorisation formula above indicates, we have to evaluate spin-correlated matrix elements. In the term where they are contracted with the metric tensor $g^{\mu \nu}$, the result corresponds to an uncorrelated matrix element via the polarisation sum. This leads to

$$
\left\langle\mathcal{M}_{b \bar{b} g, \mu}^{(0)}\left|\left(-g^{\mu \nu}\right)\right| \mathcal{M}_{b \bar{b} g, \nu}^{(0)}\right\rangle=\left|\mathcal{M}_{b \bar{b} g}^{(0)}\right|^{2} .
$$

For four-dimensional matrix elements, the product $k_{\perp}^{\mu}\left|\mathcal{M}_{b \bar{b} g, \nu}^{(0)}\right\rangle$ can be linked to helicity amplitudes using the fact that the perpendicular vector $k_{\perp}^{\mu}$ may be decomposed as

$$
k_{\perp}^{\mu}=-\sum_{\lambda}\left(\varepsilon\left(q_{4}, \lambda\right) \cdot k_{\perp}\right)\left(\varepsilon^{\mu}\left(q_{4}, \lambda\right)\right)^{*} .
$$

This is true since, by construction, $k_{\perp}^{\mu}$ lies in the plane perpendicular to the momentum of a gluon, $q_{4}$, which is spanned by the polarisation vectors $\varepsilon^{\mu}\left(q_{4}, \lambda\right)$.

\section{C.2 Single-soft factorisation (tree-level)}

In the single-soft limits, the factorisation formulae for the $H \rightarrow b \bar{b} g$ and $H \rightarrow b \bar{b} g g$ squared matrix elements are given by

$$
\begin{aligned}
& S_{4}\left|\mathcal{M}_{b \bar{b} g}^{(0)}\right|^{2}=-g_{s}^{2} C_{F}\left(\mathcal{S}_{22,4}^{(0)}-2 \mathcal{S}_{23,4}^{(0)}+\mathcal{S}_{33,4}^{(0)}\right)\left|\mathcal{M}_{b \bar{b}}^{(0)}\right|^{2}, \\
& S_{5}\left|\mathcal{M}_{b \bar{b} g g}^{(0)}\right|^{2}=-g_{s}^{2}[ C_{F}\left(\mathcal{S}_{22,5}^{(0)}-2 \mathcal{S}_{23,5}^{(0)}+\mathcal{S}_{33,5}^{(0)}\right) \\
&\left.\quad C_{A}\left(\mathcal{S}_{24,5}^{(0)}+\mathcal{S}_{34,5}^{(0)}-\mathcal{S}_{23,5}^{(0)}\right)\right]\left|\mathcal{M}_{b \bar{b} g}^{(0)}\right|^{2},
\end{aligned}
$$

where $\mathcal{S}_{i j, k}^{(0)}$ is the usual tree-level eikonal factor

$$
\mathcal{S}_{i j, k}^{(0)}=\frac{\left(q_{i} \cdot q_{j}\right)}{\left(q_{i} \cdot q_{k}\right)\left(q_{j} \cdot q_{k}\right)} .
$$

\section{C.3 Single-soft factorisation (one-loop)}

The soft limit of the one-loop amplitudes with massive quarks has been studied in refs. [89, 90]. In our case, it can be written as

$$
\begin{aligned}
S_{4} 2 \operatorname{Re}\left\langle\mathcal{M}_{b \bar{b} g}^{(0)} \mid \mathcal{M}_{b \bar{b} g}^{(1)}\right\rangle= & -g_{s}^{2} C_{F}\left(\mathcal{S}_{22,4}^{(0)}-2 \mathcal{S}_{23,4}^{(0)}+\mathcal{S}_{33,4}^{(0)}\right) \\
& \times\left[2 \operatorname{Re}\left\langle\mathcal{M}_{b \bar{b}}^{(0)} \mid \mathcal{M}_{b \bar{b}}^{(1)}\right\rangle+\left(\mathcal{R}_{23,4}^{(1)}+Z_{\alpha_{s}}^{(1)}+Z_{A}^{(1)}\right)\left|\mathcal{M}_{b \bar{b}}^{(0)}\right|^{2}\right],
\end{aligned}
$$

where in the second line we single out the contribution that comes from the renormalisation procedure, i.e. it involves terms resulting from the strong-coupling renormalisation, $Z_{\alpha_{s}}^{(1)}$, as well as a term from the gluon wave-function renormalisation, $Z_{A}^{(1)}$, see section A. Furthermore, the functions $\mathcal{R}_{i j, 4}^{(1)}$ denote the one-loop eikonal factor that can be expanded in an $\epsilon$ series as

$$
\mathcal{R}_{i j, 4}^{(1)}=4 C_{A}\left(\frac{1}{2} \mu_{R}^{2} \mathcal{S}_{i j, 4}^{(0)}\right)^{\epsilon} \sum_{k=-2}^{1} \epsilon^{k} R_{k}\left(q_{i}, q_{j} ; q_{4}\right),
$$


where functions $R_{k}\left(q_{i}, q_{j} ; q_{4}\right)$ have been calculated in ref. [90], and further simplified in ref. [91]. In our case we use the formulae from eq. (4) of ref. [91]; we emphasise that the expressions therein correspond to the unrenormalised one-loop soft-gluon current. This is particularly convenient, since we perform the renormalisation in a hybrid scheme, as outlined in section A.

Finally, we note that, for our calculation, we split the one-loop matrix elements in the factorisation formula, eq. (C.12), into finite terms and terms containing poles in $\epsilon^{-1}$ using the $\mathbf{Z}$ operator. This is reflected in eqs. (4.13) and (4.14).

\section{C.4 Double-soft factorisation}

The relevant factorisation formulae for amplitudes that involve massive particles can be obtained from refs. [30, 92], see also ref. [31]. In general, the double-soft limit requires single- and double-eikonal factors and colour-correlated matrix elements. For the $H \rightarrow b \bar{b} g g$ and $H \rightarrow b \bar{b} q \bar{q}$ matrix elements, the colour-correlated matrix elements can be expressed explicitly through $\mathrm{SU}(3)$ colour factors so that we arrive at

$$
S_{45}\left|\mathcal{M}_{b \bar{b} i j}^{(0)}\right|^{2}=g_{s}^{4} \operatorname{DSoft}_{i j}^{(0)}\left(q_{2}, q_{3} ; q_{4}, q_{5}\right)\left|\mathcal{M}_{b \bar{b}}^{(0)}\right|^{2},
$$

where $\operatorname{DSoft}_{i j}^{(0)}\left(q_{2}, q_{3} ; q_{4}, q_{5}\right)$ denotes the double-soft functions for the partons $i j \in\{g g, q \bar{q}\}$. They are given by

$$
\begin{aligned}
\operatorname{DSoft}_{g g}^{(0)}\left(q_{2}, q_{3} ; q_{4}, q_{5}\right)= & C_{F}^{2}\left(\mathcal{S}_{22,4}^{(0)}-2 \mathcal{S}_{23,4}^{(0)}+\mathcal{S}_{33,4}^{(0)}\right)\left(\mathcal{S}_{22,5}^{(0)}-2 \mathcal{S}_{23,5}^{(0)}+\mathcal{S}_{33,5}^{(0)}\right) \\
& -C_{A} C_{F}\left(\mathcal{S}_{22,45}^{g g}-\mathcal{S}_{23,45}^{g g}-\mathcal{S}_{32,45}^{g g}+\mathcal{S}_{33,45}^{g g}\right) \\
\operatorname{DSoft}_{q \bar{q}}^{(0)}\left(q_{2}, q_{3} ; q_{4}, q_{5}\right)= & C_{F} T_{F}\left(\mathcal{S}_{22,45}^{q \bar{q}}-\mathcal{S}_{23,45}^{q \bar{q}}-\mathcal{S}_{32,45}^{q \bar{q}}+\mathcal{S}_{33,45}^{q \bar{q}}\right)
\end{aligned}
$$

where $\mathcal{S}_{i j, k}^{(0)}$ is the usual single-eikonal factor, defined in eq. (C.11), and $\mathcal{S}_{i j, 45}^{g g}$ and $\mathcal{S}_{i j, 45}^{q \bar{q}}$ are the double-eikonal factors. For the $g g$ emission case, we have

$$
\mathcal{S}_{i j, 45}^{g g}=\mathcal{S}_{i j, 45}^{(m=0)}+m_{i}^{2} \mathcal{S}_{i j, 45}^{(m \neq 0)}+m_{j}^{2} \mathcal{S}_{j i, 45}^{(m \neq 0)}
$$

where [92]

$$
\begin{aligned}
\mathcal{S}_{i j, 45}^{(m=0)}= & \frac{(1-\epsilon)}{\left(q_{4} \cdot q_{5}\right)^{2}} \frac{\left(q_{i} \cdot q_{4}\right)\left(q_{j} \cdot q_{5}\right)+\left(q_{i} \cdot q_{5}\right)\left(q_{j} \cdot q_{4}\right)}{\left(q_{i} \cdot q_{45}\right)\left(q_{j} \cdot q_{45}\right)} \\
& -\frac{1}{2} \frac{\left(q_{i} \cdot q_{j}\right)}{\left(q_{i} \cdot q_{4}\right)\left(q_{j} \cdot q_{4}\right)} \frac{\left(q_{i} \cdot q_{j}\right)}{\left(q_{i} \cdot q_{5}\right)\left(q_{j} \cdot q_{5}\right)}\left[2-\frac{\left(q_{i} \cdot q_{4}\right)\left(q_{j} \cdot q_{5}\right)+\left(q_{i} \cdot q_{5}\right)\left(q_{j} \cdot q_{4}\right)}{\left(q_{i} \cdot q_{45}\right)\left(q_{j} \cdot q_{45}\right)}\right] \\
& +\frac{1}{\left(q_{4} \cdot q_{5}\right)}\left[\frac{\left(q_{i} \cdot q_{j}\right)}{\left(q_{i} \cdot q_{4}\right)\left(q_{j} \cdot q_{5}\right)}+\frac{\left(q_{i} \cdot q_{j}\right)}{\left(q_{i} \cdot q_{5}\right)\left(q_{j} \cdot q_{4}\right)}\right. \\
& \left.\quad-\frac{\left(q_{i} \cdot q_{j}\right)}{\left(q_{i} \cdot q_{45}\right)\left(q_{j} \cdot q_{45}\right)}\left(2+\frac{\left[\left(q_{i} \cdot q_{4}\right)\left(q_{j} \cdot q_{5}\right)+\left(q_{i} \cdot q_{5}\right)\left(q_{j} \cdot q_{4}\right)\right]^{2}}{2\left(q_{i} \cdot q_{4}\right)\left(q_{j} \cdot q_{5}\right)\left(q_{i} \cdot q_{5}\right)\left(q_{j} \cdot q_{4}\right)}\right)\right]
\end{aligned}
$$

and $[30]$

$$
\begin{aligned}
\mathcal{S}_{i j, 45}^{(m \neq 0)}= & -\frac{1}{4\left(q_{i} \cdot q_{4}\right)\left(q_{i} \cdot q_{5}\right)\left(q_{4} \cdot q_{5}\right)}+\frac{1}{2} \frac{\left(q_{i} \cdot q_{j}\right)}{\left(q_{i} \cdot q_{4}\right)\left(q_{i} \cdot q_{5}\right)\left(q_{j} \cdot q_{4}\right)\left(q_{j} \cdot q_{5}\right)} \frac{\left(q_{j} \cdot q_{45}\right)}{\left(q_{i} \cdot q_{45}\right)} \\
& -\frac{1}{2} \frac{1}{\left(q_{4} \cdot q_{5}\right)\left(q_{i} \cdot q_{45}\right)\left(q_{j} \cdot q_{45}\right)}\left(\frac{\left(q_{j} \cdot q_{4}\right)^{2}}{\left(q_{i} \cdot q_{4}\right)\left(q_{j} \cdot q_{5}\right)}+\frac{\left(q_{j} \cdot q_{5}\right)^{2}}{\left(q_{i} \cdot q_{5}\right)\left(q_{j} \cdot q_{4}\right)}\right) .
\end{aligned}
$$


In eqs. (C.18) and (C.19) the shorthand $q_{45}=\left(q_{4}+q_{5}\right)$ is used. For the case of a soft $q \bar{q}$ pair emission, the double-eikonal factor is given by [92]

$$
\mathcal{S}_{i j, 45}^{q \bar{q}}=\frac{1}{\left(q_{4} \cdot q_{5}\right)^{2}} \frac{\left(q_{i} \cdot q_{4}\right)\left(q_{j} \cdot q_{5}\right)+\left(q_{i} \cdot q_{5}\right)\left(q_{j} \cdot q_{4}\right)-\left(q_{i} \cdot q_{j}\right)\left(q_{4} \cdot q_{5}\right)}{\left(q_{i} \cdot q_{45}\right)\left(q_{j} \cdot q_{45}\right)} .
$$

In the strongly-ordered double-soft limit, $S_{5} S_{45}$, where we take both $\xi_{1} \rightarrow 0$ and $\xi_{2} \rightarrow 0$, the double-soft function simplifies to

$$
\begin{aligned}
S_{5} \mathrm{DSoft}_{g g}^{(0)}\left(q_{2}, q_{3} ; q_{4}, q_{5}\right)= & C_{F}^{2}\left(\mathcal{S}_{22,4}^{(0)}-2 \mathcal{S}_{23,4}^{(0)}+\mathcal{S}_{33,4}^{(0)}\right)\left(\mathcal{S}_{22,5}^{(0)}-2 \mathcal{S}_{23,5}^{(0)}+\mathcal{S}_{33,5}^{(0)}\right) \\
& +C_{F} C_{A}\left(\mathcal{S}_{22,4}^{(0)}-2 \mathcal{S}_{23,4}^{(0)}+\mathcal{S}_{33,4}^{(0)}\right)\left(\mathcal{S}_{23,5}^{(0)}-\mathcal{S}_{24,5}^{(0)}-\mathcal{S}_{34,5}^{(0)}\right) .
\end{aligned}
$$

The corresponding limit for the case of $q \bar{q}$ emission is regular.

Moreover, we need the double-soft single-collinear limit, $S_{45} C_{45}$, which can be obtained by taking the collinear limit of the double-soft function. However, a simpler expression arises if we use an iterated factorisation formula, taking first the collinear $\left(q_{4} \| q_{5}\right)$ and then the soft limit of the parent parton of the splitting $\left(q_{45}^{0} \rightarrow 0\right)$. We obtain

$$
S_{45} C_{45}\left|\mathcal{M}_{b \bar{b} i j}^{(0)}\right|^{2}=\frac{g_{s}^{4}}{\left(q_{4} \cdot q_{5}\right)} \mathcal{P}_{i j}^{(0), \mu \nu} C_{F}\left(\mathcal{S}_{22,(45), \mu \nu}^{(0)}-2 \mathcal{S}_{23,(45), \mu \nu}^{(0)}+\mathcal{S}_{33,(45), \mu \nu}^{(0)}\right)\left|\mathcal{M}_{b \bar{b}}^{(0)}\right|^{2},
$$

where we use the shorthand notation

$$
\mathcal{S}_{i j,(45), \mu \nu}^{(0)}=\frac{q_{i, \mu} q_{j, \nu}}{\left(q_{i} \cdot q_{45}\right)\left(q_{45} \cdot q_{j}\right)} .
$$

\section{Integrated subtraction terms}

In this section we report formulae for integrated subtraction terms that we use throughout the calculation. For the convenience of the reader, we also include results available in the literature.

\section{D.1 Single-collinear subtraction terms}

The relevant factorisation formula for the single-collinear limit is given in eq. (C.2). For the integrated subtraction terms, we integrate the splitting function over the unresolved phase space in $d$ dimensions.

We recall that the splitting functions in eqs. (C.4) and (C.5) contain a term proportional to $k_{\perp}^{\mu} k_{\perp}^{\nu} / k_{\perp}^{2}$ which is contracted with the spin-correlated matrix element $\left\langle\mathcal{M}_{b \bar{b} g, \mu}^{(0)} \mid \mathcal{M}_{b \bar{b} g, \nu}^{(0)}\right\rangle$. In contrast to the single-collinear subtraction terms, where the spin correlations are required to make the subtraction local, the integrated subtraction term can be averaged over the azimuthal directions of momentum $q_{5}$. The reduced matrix element depends only on the sum of momenta, $q_{45}=\left(q_{4}+q_{5}\right)$, which is independent of the azimuthal direction of $q_{5}$. Therefore, the integral over $\mathrm{d} \Omega_{5}^{(2-2 \epsilon)}$ decouples and yields

$$
\left(\int \mathrm{d} \Omega_{5}^{(2-2 \epsilon)}\right)^{-1} \int \mathrm{d} \Omega_{5}^{(2-2 \epsilon)} \frac{k_{\perp}^{\mu} k_{\perp}^{\nu}}{k_{\perp}^{2}}=\frac{1}{2(1-\epsilon)}\left[g^{\mu \nu}-\frac{n^{\mu} \bar{n}^{\nu}+\bar{n}^{\mu} n^{\nu}}{(n \cdot \bar{n})}\right]
$$


with $\bar{n}^{\mu}=n_{\nu}$ and $n^{\mu}=q_{4}^{\mu} / q_{4}^{0}$ in the collinear limit. To derive this result, we use the fact that the right-hand side of eq. (D.1) is invariant under rotations in the $(2-2 \epsilon)$ dimensional sphere, that it has to be orthogonal to $q_{4}^{\mu}$ and that by definition the time components $(\mu=0$ and $\nu=0)$ need to vanish [31]. Note that, due to the Ward identity $q_{4}^{\mu}\left|\mathcal{M}_{b \bar{b} g, \mu}^{(0)}\right\rangle=0$, the $n^{\mu} \bar{n}^{\nu}$ and $\bar{n}^{\mu} n^{\nu}$ terms in eq. (D.1) drop out when contracted with the spin-correlated squared matrix elements. ${ }^{5}$

This means that after azimuthal averaging and using eq. (C.7) we replace

$$
\frac{k_{\perp}^{\mu} k_{\perp}^{\nu}}{k_{\perp}^{2}}\left\langle\mathcal{M}_{b \bar{b} g, \mu}^{(0)} \mid \mathcal{M}_{b \bar{b} g, \nu}^{(0)}\right\rangle \rightarrow \quad-\frac{1}{2(1-\epsilon)}\left|\mathcal{M}_{b \bar{b} g}^{(0)}\right|^{2}
$$

As a result the factorisation formula of eq. (C.2) simplifies to

$$
C_{45}\left|\mathcal{M}_{b \bar{b} i j}^{(0)}\right|^{2}=\frac{g_{s}^{2}}{\left(q_{4} \cdot q_{5}\right)}\left\langle\mathcal{P}_{i j}^{(0)}(z ; \epsilon)\right\rangle\left|\mathcal{M}_{b \bar{b} g}^{(0)}\right|^{2}
$$

where $\left\langle\mathcal{P}_{i j}^{(0)}(z ; \epsilon)\right\rangle$ are the azimuthally averaged splitting functions and read

$$
\begin{aligned}
\left\langle\mathcal{P}_{g g}^{(0)}(z ; \epsilon)\right\rangle & =2 C_{A}\left[\frac{z}{1-z}+\frac{1-z}{z}+z(1-z)\right], \\
\left\langle\mathcal{P}_{q \bar{q}}^{(0)}(z ; \epsilon)\right\rangle & =T_{F}\left[1-\frac{2 z(1-z)}{1-\epsilon}\right] .
\end{aligned}
$$

To obtain the integrated collinear subtraction term, we perform the integral over the unresolved phase space, $\left[\mathrm{d} q_{5}\right]$, using the parametrisation of eq. (2.31) in the collinear limit. We arrive at

$$
C_{45} \int \mathrm{d} \Phi_{b \bar{b} g g}^{E_{4}>E_{5}}\left(q_{1}\right)\left|\mathcal{M}_{b \bar{b} i j}^{(0)}\right|^{2}=g_{s}^{2} \mathcal{P}_{i j \text {,int }}^{(0)} \int \mathrm{d} \Phi_{b \bar{b} g}\left(q_{1}\right)\left(\xi_{1} / 2\right)^{-2 \epsilon}\left|\mathcal{M}_{b \bar{b} g}^{(0)}\right|^{2},
$$

with the integrated splitting function $\mathcal{P}_{i j \text {,int }}^{(0)}$ defined as

$$
\begin{aligned}
\mathcal{P}_{i j \text {,int }}^{(0)} & =\left(\mu_{R}^{2}\right)^{\epsilon} S_{\epsilon} E_{\max }^{2-2 \epsilon}\left(\frac{\xi_{1}}{2}\right)^{2} \int \frac{\mathrm{d} \Omega_{5}^{(2-2 \epsilon)}}{2(2 \pi)^{3-2 \epsilon}} \int_{0}^{1} \frac{\mathrm{d} \eta}{\eta^{\epsilon}} \int_{0}^{1} \mathrm{~d} \xi_{2}\left(\xi_{2}\left(2-\xi_{2}\right)\right)^{1-2 \epsilon} \frac{\left\langle\mathcal{P}_{i j}^{(0)}\left(1-\frac{\xi_{2}}{2} ; \epsilon\right)\right\rangle}{\left(q_{4} \cdot q_{5}\right)} \\
& =\frac{1}{2}\left(\mu_{R}^{2}\right)^{\epsilon} S_{\epsilon} E_{\max }^{-2 \epsilon} \int \frac{\mathrm{d} \Omega_{5}^{(2-2 \epsilon)}}{2(2 \pi)^{3-2 \epsilon}} \int_{0}^{1} \frac{\mathrm{d} \eta}{\eta^{1+\epsilon}} \int_{0}^{1} \mathrm{~d} \xi_{2}\left(\xi_{2}\left(2-\xi_{2}\right)\right)^{-2 \epsilon}\left\langle\mathcal{P}_{i j}^{(0)}\left(1-\frac{\xi_{2}}{2} ; \epsilon\right)\right\rangle .
\end{aligned}
$$

To perform the integral in eq. (D.7) we use the fact that the integrand is symmetric under $\xi_{2} \leftrightarrow\left(2-\xi_{2}\right)$ exchange and hence we can extend the integration region to $0<\xi_{2}<2$ at the cost of introducing a factor of $1 / 2$. Then we obtain

$$
\begin{aligned}
& \mathcal{P}_{g g, \text { int }}^{(0)}=\left(\frac{\mu_{R}^{2}}{16 E_{\max }^{2}}\right)^{\epsilon} \frac{C_{A}}{(4 \pi)^{2}} \frac{6(2-3 \epsilon)}{\epsilon^{2}} \frac{e^{\epsilon \gamma_{\mathrm{E}}} \Gamma^{2}(2-2 \epsilon)}{\Gamma(4-4 \epsilon) \Gamma(1-\epsilon)}, \\
& \mathcal{P}_{q \bar{q}, \text { int }}^{(0)}=\left(\frac{\mu_{R}^{2}}{16 E_{\max }^{2}}\right)^{\epsilon} \frac{T_{F}}{(4 \pi)^{2}} \frac{\epsilon(10-8 \epsilon)-4}{\epsilon} \frac{e^{\epsilon \gamma_{\mathrm{E}}} \Gamma(1-2 \epsilon) \Gamma(2-2 \epsilon)}{\Gamma(4-4 \epsilon) \Gamma(2-\epsilon)} .
\end{aligned}
$$

\footnotetext{
${ }^{5}$ Note that this argument is not necessarily valid if more that one gluon is spin-correlated in the reduced amplitude.
} 
In the case of $\mathcal{P}_{g g, \text { int }}^{(0)}$, it is necessary to also calculate the integrated splitting function in the $\xi_{2} \rightarrow 0$ limit. We find

$$
\mathcal{P}_{g g, \text { int }}^{(0), \text { soft }}=\left(\frac{\mu_{R}^{2}}{4 E_{\max }^{2}}\right)^{\epsilon} \frac{C_{A}}{(4 \pi)^{2}} \frac{2}{\epsilon^{2}} \frac{e^{\epsilon \gamma_{\mathrm{E}}}}{\Gamma(1-\epsilon)} .
$$

\section{D.2 Single-soft subtraction terms (tree-level)}

When considering a single-soft emission of a gluon with momentum $q_{k}$ that involves partons with momenta $q_{i}$ and $q_{j}$, we encounter the tree-level eikonal factor $\mathcal{S}_{i j, k}^{(0)}$, defined in eq. (C.11). In the following we will use the notation

$$
\hat{q}^{\mu}=\frac{q^{\mu}}{q^{0}}
$$

to denote a momentum rescaled by its energy component.

The soft function is integrated over the unresolved phase space using the parametrisation in eq. (2.21), where the Born phase space decouples in the soft limit. Thus, the gluon energy is unconstrained unless we insert some bound by hand. For simplicity, we keep the integration domain of $\xi_{1}$ unchanged, which corresponds to an upper limit of the energy of $E_{\text {max }}$. We arrive at

$$
\begin{aligned}
\mathcal{S}_{i j \text {,int }}^{(0)} & =\int\left[\mathrm{d} q_{4}\right] \mathcal{S}_{i j, 4}^{(0)} \\
& =\left(\frac{\mu_{R}^{2}}{E_{\max }^{2}}\right)^{\epsilon} S_{\epsilon} \int_{0}^{1} \frac{\mathrm{d} \xi_{1}}{\xi_{1}^{1+2 \epsilon}} \int \frac{\mathrm{d} \Omega_{4}^{(3-2 \epsilon)}}{2(2 \pi)^{3-2 \epsilon}} \frac{\left(q_{i} \cdot q_{j}\right)}{\left(q_{i} \cdot \hat{q}_{4}\right)\left(\hat{q}_{4} \cdot q_{j}\right)} \\
& =\left(\frac{\mu_{R}^{2}}{E_{\max }^{2}}\right)^{\epsilon} S_{\epsilon}\left[-\frac{1}{2 \epsilon}\right] \int \frac{\mathrm{d} \Omega_{4}^{(3-2 \epsilon)}}{2(2 \pi)^{3-2 \epsilon}} \frac{\left(q_{i} \cdot q_{j}\right)}{\left(q_{i} \cdot \hat{q}_{4}\right)\left(\hat{q}_{4} \cdot q_{j}\right)},
\end{aligned}
$$

where the pole in the last line arises from performing the energy integral. We are left with angular integrals only, for which we write

$$
\begin{aligned}
\int \mathrm{d} \Omega_{4}^{(3-2 \epsilon)} \frac{\left(q_{i} \cdot q_{j}\right)}{\left(q_{i} \cdot \hat{q}_{4}\right)\left(\hat{q}_{4} \cdot q_{j}\right)} & =\int \mathrm{d} \Omega_{4}^{(1-2 \epsilon)} \int \mathrm{d}(\cos \theta) \mathrm{d} \phi(\sin \theta \sin \phi)^{-2 \epsilon} \frac{\left(q_{i} \cdot q_{j}\right)}{\left(q_{i} \cdot \hat{q}_{4}\right)\left(\hat{q}_{4} \cdot q_{j}\right)} \\
& =\frac{\Gamma(1-\epsilon)}{(4 \pi)^{\epsilon} \Gamma(1-2 \epsilon)}(2 \pi) I\left(q_{i}, q_{j}\right) .
\end{aligned}
$$

Here, we introduce the auxiliary function

$$
I\left(q_{i}, q_{j}\right)=\int \mathrm{d}(\cos \theta) \int \frac{\mathrm{d} \phi}{\pi}(\sin \theta \sin \phi)^{-2 \epsilon} \frac{\left(q_{i} \cdot q_{j}\right)}{\left(q_{i} \cdot \hat{q}_{4}\right)\left(\hat{q}_{4} \cdot q_{j}\right)} .
$$

We write it as a Laurent series in $\epsilon$, i.e.

$$
I\left(q_{i}, q_{j}\right)=\sum_{k=-1}^{2} \epsilon^{k} I^{(k)}\left(q_{i}, q_{j}\right)+\mathcal{O}\left(\epsilon^{3}\right),
$$

where the coefficients $I^{(k)}\left(q_{i}, q_{j}\right)$ depend on the momenta $q_{i}$ and $q_{j}$, in particular on whether they are massless or massive. All necessary coefficients for the single-soft integrated subtraction terms, except for the $\mathcal{O}\left(\epsilon^{2}\right)$ terms, have been obtained in ref. [93], see appendix A 
therein. For completeness, we collect below the formulae for those $I^{(k)}\left(q_{i}, q_{j}\right)$ which are relevant to our calculation.

We write the full integrated single-soft eikonal factor as

$$
\mathcal{S}_{i j \text {,int }}^{(0)}=-\frac{1}{(4 \pi)^{2}} \frac{1}{\epsilon} \frac{e^{\epsilon \gamma_{\mathrm{E}}} \Gamma(1-\epsilon)}{\Gamma(1-2 \epsilon)}\left(\frac{\mu_{R}^{2}}{4 E_{\max }^{2}}\right)^{\epsilon} I\left(q_{i}, q_{j}\right) .
$$

We have to distinguish the case where the emitters are two massive particles, which we denote by a subscript MM, and the case where emitter $i$ is massive and emitter $j$ is massless, which we denote by M0. For our calculation, we need the coefficients $I_{\mathrm{MM}}^{(k)}\left(q_{i}, q_{j}\right)$ and $I_{\mathrm{M} 0}^{(k)}\left(q_{i}, q_{j}\right)$ for $k \in\{-1,0,1\}$ when the directions of the momenta $q_{i}$ and $q_{j}$ are arbitrary. Moreover, we need $I_{\mathrm{MM}}^{(k)}\left(q_{i}, q_{j}\right)$ for $k \in\{-1,0,1,2\}$ in two special cases: for the case where the two momenta are equal, $q_{i}=q_{j}$ and for the case where $q_{i}$ and $q_{j}$ are in a back-to-back configuration.

Two massive emitters. The reported formulae correspond to the result outlined in eqs. (A.41) to (A.51) of ref. [93]. We consider two time-like momenta $q_{i}^{2}=m_{i}^{2}$ and $q_{j}^{2}=m_{j}^{2}$. With the definition of $v_{i j}$ from eq. (B.3) and the notation

$$
\vec{u}=\frac{\vec{q}_{i}}{E_{i}}, \quad \text { and } \quad \vec{w}=\frac{\vec{q}_{j}}{E_{j}},
$$

we introduce the shorthand notations

$$
\begin{array}{ll}
A^{2}=(\vec{u} \cdot \vec{u})+(\vec{w} \cdot \vec{w})-2(\vec{u} \cdot \vec{w}), & X_{1}=(\vec{u} \cdot \vec{u})-(\vec{u} \cdot \vec{w}), \\
B^{2}=(\vec{u} \cdot \vec{u})(\vec{w} \cdot \vec{w})-(\vec{u} \cdot \vec{w})^{2}, & X_{2}=(\vec{w} \cdot \vec{w})-(\vec{u} \cdot \vec{w}) .
\end{array}
$$

Furthermore, we need the following arguments

$$
\begin{array}{ll}
z_{+}=A+\sqrt{A^{2}-B^{2}}, & z_{1}=\sqrt{X_{1}^{2}+B^{2}}-X_{1}, \\
z_{-}=A-\sqrt{A^{2}-B^{2}}, & z_{2}=\sqrt{X_{2}^{2}+B^{2}}+X_{2},
\end{array}
$$

which will be used in the function

$$
\begin{aligned}
K(z)= & -2 \operatorname{Li}_{2}\left(\frac{2 z_{-}\left(z_{+}-z\right)}{\left(z_{+}-z_{-}\right)\left(z_{-}+z\right)}\right)-2 \operatorname{Li}_{2}\left(\frac{-2 z_{+}\left(z_{-}+z\right)}{\left(z_{+}-z_{-}\right)\left(z_{+}-z\right)}\right) \\
& -\frac{1}{2} \log ^{2}\left(\frac{\left(z-z_{-}\right)\left(z_{+}-z\right)}{\left(z+z_{-}\right)\left(z_{+}+z\right)}\right) .
\end{aligned}
$$

With these abbreviations, the coefficients of the massive-massive angular integral read

$$
\begin{aligned}
I_{\mathrm{MM}}^{(-1)}\left(q_{i}, q_{j}\right) & =0 \\
I_{\mathrm{MM}}^{(0)}\left(q_{i}, q_{j}\right) & =\frac{1}{v_{i j}} \log \left(\frac{1+v_{i j}}{1-v_{i j}}\right), \\
I_{\mathrm{MM}}^{(1)}\left(q_{i}, q_{j}\right) & =\frac{1-(\vec{u} \cdot \vec{w})}{\sqrt{A^{2}-B^{2}}}\left(K\left(z_{2}\right)-K\left(z_{1}\right)\right) .
\end{aligned}
$$


One massive and one massless emitter. The reported formulae correspond to the result outlined in eqs. (A.22) to (A.24) of ref. [93]. We consider one time-like momentum $q_{i}^{2}=m_{i}{ }^{2}$ and one light-like momentum $q_{j}^{2}=0$. We define the symbol

$$
\kappa=\sqrt{1-\frac{m_{i}^{2}}{E_{i}^{2}}} .
$$

Then the coefficients of the massive-massless angular integral are

$$
\begin{aligned}
I_{\mathrm{M} 0}^{(-1)}\left(q_{i}, q_{j}\right)=-1 & \\
I_{\mathrm{M} 0}^{(0)}\left(q_{i}, q_{j}\right)=\log & \left(\frac{\left(\hat{q}_{i} \cdot \hat{q}_{j}\right)^{2}}{\left(\hat{q}_{i} \cdot \hat{q}_{i}\right)}\right), \\
I_{\mathrm{M} 0}^{(1)}\left(q_{i}, q_{j}\right)=-2 & \left\{\frac{1}{4} \log ^{2}\left(\frac{1-\kappa}{1+\kappa}\right)+\log \left(\frac{\left(\hat{q}_{i} \cdot \hat{q}_{j}\right)}{1+\kappa}\right) \log \left(\frac{\left(\hat{q}_{i} \cdot \hat{q}_{j}\right)}{1-\kappa}\right)\right. \\
+ & \left.\operatorname{Li}_{2}\left(1-\frac{\left(\hat{q}_{i} \cdot \hat{q}_{j}\right)}{1+\kappa}\right)+\operatorname{Li}_{2}\left(1-\frac{\left(\hat{q}_{i} \cdot \hat{q}_{j}\right)}{1-\kappa}\right)\right\} .
\end{aligned}
$$

Two massive back-to-back emitters. We consider the special case of two massive emitters with the same mass, $q_{i}^{2}=m^{2}$ and $q_{j}^{2}=m^{2}$, arranged in a back-to-back configuration, $\vec{q}_{i}=-\vec{q}_{j}$. We then have $E_{i}=E_{j}=M_{H} / 2$ and, therefore, $\kappa=\beta$. The expansion coefficients are given by

$$
\begin{aligned}
& I_{\mathrm{MM}, \mathrm{b} 2 \mathrm{~b}}^{(-1)}\left(q_{i}, q_{j}\right)=0, \\
& I_{\mathrm{MM}, \mathrm{b} 2 \mathrm{~b}}^{(0)}\left(q_{i}, q_{j}\right)=-\frac{1+\beta^{2}}{\beta} \log \left(\frac{1-\beta}{1+\beta}\right), \\
& I_{\mathrm{MM}, \mathrm{b} 2 \mathrm{~b}}^{(1)}\left(q_{i}, q_{j}\right)=\frac{1+\beta^{2}}{\beta}\left\{\operatorname{Li}_{2}\left(\frac{2 \beta}{1+\beta}\right)-\operatorname{Li}_{2}\left(-\frac{2 \beta}{1-\beta}\right)\right\}, \\
& I_{\mathrm{MM}, \mathrm{b} 2 \mathrm{~b}}^{(2)}\left(q_{i}, q_{j}\right)=\frac{1+\beta^{2}}{\beta}\left\{-\frac{1}{3} \log ^{3}\left(\frac{1-\beta}{1+\beta}\right)+2 \operatorname{Li}_{3}\left(\frac{2 \beta}{1+\beta}\right)-2 \operatorname{Li}_{3}\left(-\frac{2 \beta}{1-\beta}\right)\right. \\
&\left.\quad-\log \left(\frac{1-\beta}{1+\beta}\right)\left[\operatorname{Li}_{2}\left(\frac{2 \beta}{1+\beta}\right)+\operatorname{Li}_{2}\left(-\frac{2 \beta}{1-\beta}\right)\right]\right\},
\end{aligned}
$$

where the subscript "b2b" indicates a back-to-back configuration of the emitters. Formulae for $I_{\mathrm{MM}, \mathrm{b} 2 \mathrm{~b}}^{(k)}$ for $k \in\{-1,0,1\}$ can be obtained from eqs. (D.21) to (D.23) by taking the relevant limit. The formula for $I_{\mathrm{MM}, \mathrm{b} 2 \mathrm{~b}}^{(2)}$ was calculated independently; this $\mathcal{O}\left(\epsilon^{2}\right)$ term is needed in the soft limit of the real-virtual contribution.

Self-correlated massive emitter. We consider the special case of a self-correlated massive emitter, $i=j$, for a time-like momentum $q_{i}$ with $q_{i}^{2}=m^{2}$. Then we have

$$
\begin{aligned}
& I_{\mathrm{MM}}^{(-1)}\left(q_{i}, q_{i}\right)=0, \\
& I_{\mathrm{MM}}^{(0)}\left(q_{i}, q_{i}\right)=2, \\
& I_{\mathrm{MM}}^{(1)}\left(q_{i}, q_{i}\right)=-\frac{2}{\kappa} \log \left(\frac{1-\kappa}{1+\kappa}\right), \\
& I_{\mathrm{MM}}^{(2)}\left(q_{i}, q_{i}\right)=\frac{2}{\kappa}\left\{\operatorname{Li}_{2}\left(\frac{2 \kappa}{1+\kappa}\right)-\mathrm{Li}_{2}\left(-\frac{2 \kappa}{1-\kappa}\right)\right\} .
\end{aligned}
$$

The $\mathcal{O}\left(\epsilon^{2}\right)$ term was calculated independently. 


\section{D.3 Single-soft subtraction terms (one-loop)}

We consider the soft limit of the one-loop $H \rightarrow b \bar{b} g$ amplitude which is given in eq. (C.12). This factorisation involves both tree-level and one-loop soft functions. The integration of the tree-level eikonal factors is discussed in section D.2, while in this section we focus on the integration of those terms in the one-loop soft function of eq. (C.12) which contain $\mathcal{R}_{i j, 4}^{(1)}$.

We integrate the one-loop eikonal factors over the soft-gluon phase space, i.e.

$$
\mathcal{R}_{\text {int }}^{(1)}=-\int\left[\mathrm{d} q_{4}\right]\left(\mathcal{S}_{22,4}^{(0)}-2 \mathcal{S}_{23,4}^{(0)}+\mathcal{S}_{33,4}^{(0)}\right) \mathcal{R}_{23,4}^{(1)},
$$

where $\mathcal{S}_{i j, 4}^{(0)}$ and $\mathcal{R}_{23,4}^{(1)}$ are defined in eqs. (C.11) and (C.13), respectively. Since only $\mathcal{R}_{23,4}^{(1)}$ appears, we restrict ourselves to the case of two emitters with the same non-vanishing mass in a back-to-back configuration.

The soft-gluon phase space is parametrised using eq. (2.21). Note that even though the soft-gluon momentum factorises from the energy-momentum conserving $\delta$-function, we restrict ourselves to the same integration region as stated in eq. (2.21); this is in accordance with the choice made for the integrated tree-level eikonal factor. The only non-trivial integral is the integration over the angle $\theta$ between the momentum of the soft gluon $q_{4}$ and the $b$-quark momentum $q_{i}$. We reexpress this angle in terms of the variable $\lambda=\cos \theta$ and use the symmetry under $\lambda \rightarrow-\lambda$ to restrict the domain of integration to $\lambda \in[0,1]$. We arrive at

$$
\int\left[\mathrm{d} q_{4}\right]=\frac{\mu_{R}^{2 \epsilon} S_{\epsilon} E_{\max }^{2-2 \epsilon}}{(2 \pi)^{3-2 \epsilon}} \int \mathrm{d} \Omega_{4}^{(2-2 \epsilon)} \int_{0}^{1} \mathrm{~d} \xi \xi^{1-2 \epsilon} \int_{0}^{1} \mathrm{~d} \lambda\left(1-\lambda^{2}\right)^{-\epsilon} .
$$

The energy dependence factorises from the integrand, which means that the integral over $\mathrm{d} \xi$ can be solved trivially. The angular integral $\mathrm{d} \Omega_{4}^{(2-2 \epsilon)}$ is performed using eq. (2.22).

The expressions for the expansion coefficients $R_{k}\left(q_{i}, q_{j} ; q_{4}\right)$ in ref. [91] are given in terms of the variables

$$
\begin{aligned}
& \alpha_{i}=\frac{m_{i}^{2}}{2} \frac{\left(q_{j} \cdot q_{4}\right)}{\left(q_{i} \cdot q_{4}\right)\left(q_{i} \cdot q_{j}\right)}=\frac{\left(1-\beta^{2}\right)(1+\beta \lambda)}{2\left(1+\beta^{2}\right)(1-\beta \lambda)}, \\
& \alpha_{j}=\frac{m_{j}^{2}}{2} \frac{\left(q_{i} \cdot q_{4}\right)}{\left(q_{j} \cdot q_{4}\right)\left(q_{i} \cdot q_{j}\right)}=\frac{\left(1-\beta^{2}\right)(1-\beta \lambda)}{2\left(1+\beta^{2}\right)(1+\beta \lambda)},
\end{aligned}
$$

and contain at most classical polylogarithms with arguments composed of these variables. We rewrite those special functions in terms of iterated integrals of argument $\lambda$ over the alphabet

$$
\frac{\mathrm{d} \lambda}{\lambda}, \quad \frac{\mathrm{d} \lambda}{1+\lambda}, \quad \frac{\mathrm{d} \lambda}{1-\lambda}, \quad \frac{\mathrm{d} \lambda}{1+\beta \lambda}, \quad \frac{\mathrm{d} \lambda}{1-\beta \lambda} .
$$

As the rational coefficients in front of the iterated integrals also only contain these letters, the integration over $\lambda$ can again be performed in terms of iterated integrals over the same alphabet. These iterated integrals are then evaluated at 1 and we rewrite them in terms of harmonic polylogarithms [67] of argument $\beta$ with up to weight four. All manipulations of the iterated integrals are performed with the Mathematica package HarmonicSums [67, 94-103]. 
Note that the term $\mathcal{R}_{\text {int }}^{(1)}$ is again a Laurent series in $\epsilon$. Therefore, we write

$$
\mathcal{R}_{\text {int }}^{(1)}=\frac{4 C_{A}}{(4 \pi)^{2}}\left(\frac{\mu_{R}^{2}}{4 E_{\max }^{2}}\right)^{2 \epsilon} \sum_{k=-3}^{0} \mathcal{R}_{\text {int }}^{(1, k)}(\beta) \epsilon^{k} .
$$

The expansion coefficients $\mathcal{R}_{\text {int }}^{(1, k)}$ for $m_{b}=4.78 \mathrm{GeV}$ and $M_{H}=125.09 \mathrm{GeV}$ evaluate to

$$
\begin{gathered}
\mathcal{R}_{\text {int }}^{(1,-3)}(\beta)=5.52628705137596, \\
\mathcal{R}_{\text {int }}^{(1,-2)}(\beta)=35.3923534452863, \\
\mathcal{R}_{\text {int }}^{(1,-1)}(\beta)=111.992677970445, \\
\mathcal{R}_{\text {int }}^{(1,0)}(\beta)=245.654621810082 .
\end{gathered}
$$

The analytical expressions can be found in computer-readable form in the supplementary material of this article.

\section{D.4 Double-soft subtraction terms}

For the integrated subtraction terms of the double-real contribution we have to integrate the double-soft function, discussed in section C.4, over the unresolved phase space in $d$ dimensions. We define the integrated double-soft function as

$$
\operatorname{DSoft}_{i j, \text { int }}^{(0)}\left(q_{2}, q_{3}\right)=\int\left[\mathrm{d} q_{4}\right]\left[\mathrm{d} q_{5}\right] \operatorname{DSoft}_{i j}^{(0)}\left(q_{2}, q_{3} ; q_{4}, q_{5}\right)
$$

We use the phase-space parametrisation outlined in eq. (2.31), taking into account that the gluon momenta decouple from the overall energy-momentum conserving $\delta$-function. Note that the decoupling of soft particles means that, in principle, their energies are unbounded unless we introduce some constraint by hand. For simplicity, we choose to keep the bound that explicitly appears in the formulation of the phase-space measure, i.e. we keep $E_{45, \max }$ unchanged while the $\mathrm{d} \xi_{1} \mathrm{~d} \xi_{2}$ integration region still covers the unit square.

It is particularly useful to keep the Born configuration fixed so that the $b$-quark points along the $\hat{z}$-axis, i.e.

$$
q_{2}=\frac{1}{2} M_{H}\left(\hat{t}^{\mu}+\beta \hat{z}^{\mu}\right) \quad \text { and } \quad q_{3}=\frac{1}{2} M_{H}\left(\hat{t}^{\mu}-\beta \hat{z}^{\mu}\right),
$$

where $\hat{t}^{\mu}$ and $\hat{z}^{\mu}$ are unit vectors along time and $\hat{z}$ axes, respectively. After expressing the integrand using the phase-space parametrisation, the $\xi_{1}$ dependence factorises and can be integrated analytically. The remaining integrals are performed numerically.

The integrands of eq. (D.43) are still divergent in the strongly-ordered $\left(S_{5}\right)$ and collinear $\left(C_{45}\right)$ limits. These divergences can be handled using the endpoint subtraction method, as discussed in section 2.2. We write

$$
S_{45}=\left(I-S_{5}\right)\left(I-C_{45}\right) S_{45}+\left(I-S_{5}\right) C_{45} S_{45}+S_{5}\left(I-C_{45}\right) S_{45}+S_{5} C_{45} S_{45} .
$$

Note that the regularisation in the strongly-ordered limit applies only to the $g g$ emission case of eq. (C.15). The relevant subtraction terms in eq. (D.45) are constructed using the factorisation formulae from section $\mathrm{C}$. 


\begin{tabular}{|lclll|}
\hline & $k=-3$ & $k=-2$ & $k=-1$ & $k=0$ \\
\hline$C_{g g, C_{A} C_{F}}^{(k)}$ & $-22.105148(3)$ & $-120.8071(1)$ & $-337.441(1)$ & $-613.869(4)$ \\
$C_{g g, C_{F}^{2}}^{(k)}$ & - & $+244.3194(4)$ & $+915.818(2)$ & $+984.741(6)$ \\
$C_{q \bar{q}, C_{F} T_{F}}^{(k)}$ & - & $+7.368385(3)$ & $+39.9006(1)$ & $+124.966(1)$ \\
\hline
\end{tabular}

Table 6. Coefficients of the $\epsilon$ expansion of the integrated double-soft function for $g g$ channel (first two rows), and the $q \bar{q}$ channel (the last row). We use $m_{b}=4.78 \mathrm{GeV}$ and $M_{H}=125.09 \mathrm{GeV}$.

The integrated double-soft function can be written as a Laurent series, i.e.

$$
\operatorname{DSoft}_{i j, \text { int }}^{(0)}\left(q_{2}, q_{3}\right)=\frac{1}{(4 \pi)^{4}}\left(\frac{\mu_{R}^{2}}{E_{\text {max }}^{2}}\right)^{2 \epsilon} \sum_{k=-3}^{0} C_{i j}^{(k)} \epsilon^{k} .
$$

Note that the highest pole that occurs in the $g g$ channel is $\epsilon^{-3}$. This arises from taking all singular limits in the integrand, i.e. the strongly-ordered, collinear and the double-soft limits. In the $q \bar{q}$ case we only have an $\epsilon^{-2}$ pole since the strongly-ordered soft limit, $S_{5}$, is regular. Furthermore, we split the $C_{i j}^{(k)}$ coefficients into separate colour structures,

$$
\begin{aligned}
& C_{g g}^{(k)}=C_{A} C_{F} C_{g g, C_{A} C_{F}}^{(k)}+C_{F}^{2} C_{g g, C_{F}^{2}}^{(k)}, \\
& C_{q \bar{q}}^{(k)}=C_{F} T_{F} C_{q \bar{q}, C_{F} T_{F}}^{(k)} .
\end{aligned}
$$

We present numerical values for the integrated double-soft function coefficients, evaluated for $m_{b}=4.78 \mathrm{GeV}$ and $M_{H}=125.09 \mathrm{GeV}$, in table 6 .

Open Access. This article is distributed under the terms of the Creative Commons Attribution License (CC-BY 4.0), which permits any use, distribution and reproduction in any medium, provided the original author(s) and source are credited.

\section{References}

[1] ATLAS and CMS collaborations, Combined measurement of the Higgs boson mass in pp collisions at $\sqrt{s}=7$ and $8 \mathrm{TeV}$ with the ATLAS and CMS experiments, Phys. Rev. Lett. 114 (2015) 191803 [arXiv: 1503.07589] [INSPIRE].

[2] ATLAS collaboration, Observation of $H \rightarrow b \bar{b}$ decays and $V H$ production with the ATLAS detector, Phys. Lett. B 786 (2018) 59 [arXiv: 1808.08238] [INSPIRE].

[3] CMS collaboration, Observation of Higgs boson decay to bottom quarks, Phys. Rev. Lett. 121 (2018) 121801 [arXiv:1808.08242] [INSPIRE].

[4] E. Braaten and J.P. Leveille, Higgs boson decay and the running mass, Phys. Rev. D 22 (1980) 715 [INSPIRE].

[5] N. Sakai, Perturbative QCD corrections to the hadronic decay width of the Higgs boson, Phys. Rev. D 22 (1980) 2220 [inSPIRE].

[6] P. Janot, First order $Q E D$ and $Q C D$ radiative corrections to Higgs decay into massive fermions, Phys. Lett. B 223 (1989) 110 [INSPIRE]. 
[7] M. Drees and K.-I. Hikasa, Note on QCD corrections to hadronic Higgs decay, Phys. Lett. B 240 (1990) 455 [Erratum ibid. B 262 (1991) 497] [INSPIRE].

[8] A.L. Kataev and V.T. Kim, The effects of the massless $O\left(\alpha_{s}^{2}\right), O\left(\alpha \alpha_{s}\right), O\left(\alpha^{2}\right) Q C D$ and QED corrections and of the massive contributions to $\Gamma\left(H^{0} \rightarrow b \bar{b}\right)$, hep-ph/9304282 [INSPIRE].

[9] A.L. Kataev and V.T. Kim, The effects of the QCD corrections to $\Gamma\left(H^{0} \rightarrow b \bar{b}\right)$, Mod. Phys. Lett. A 9 (1994) 1309 [INSPIRE].

[10] P.A. Baikov, K.G. Chetyrkin and J.H. Kühn, Scalar correlator at $O\left(\alpha_{s}^{4}\right)$, Higgs decay into b-quarks and bounds on the light quark masses, Phys. Rev. Lett. 96 (2006) 012003 [hep-ph/0511063] [INSPIRE].

[11] R. Harlander and M. Steinhauser, Higgs decay to top quarks at $O\left(\alpha_{s}^{2}\right)$, Phys. Rev. D 56 (1997) 3980 [hep-ph/9704436] [INSPIRE].

[12] S.A. Larin, T. van Ritbergen and J.A.M. Vermaseren, The large top quark mass expansion for Higgs boson decays into bottom quarks and into gluons, Phys. Lett. B 362 (1995) 134 [hep-ph/9506465] [INSPIRE].

[13] K.G. Chetyrkin and A. Kwiatkowski, Second order QCD corrections to scalar and pseudoscalar Higgs decays into massive bottom quarks, Nucl. Phys. B 461 (1996) 3 [hep-ph/9505358] [INSPIRE].

[14] A. Primo, G. Sasso, G. Somogyi and F. Tramontano, Exact top Yukawa corrections to Higgs boson decay into bottom quarks, Phys. Rev. D 99 (2019) 054013 [arXiv:1812.07811] [INSPIRE].

[15] E. Chaubey and S. Weinzierl, Two-loop master integrals for the mixed QCD-electroweak corrections for $H \rightarrow b \bar{b}$ through a Htt-coupling, JHEP 05 (2019) 185 [arXiv:1904.00382] [INSPIRE].

[16] C. Anastasiou, F. Herzog and A. Lazopoulos, The fully differential decay rate of a Higgs boson to bottom-quarks at NNLO in QCD, JHEP 03 (2012) 035 [arXiv:1110.2368] [INSPIRE].

[17] V. Del Duca, C. Duhr, G. Somogyi, F. Tramontano and Z. Trócsányi, Higgs boson decay into b-quarks at NNLO accuracy, JHEP 04 (2015) 036 [arXiv: 1501.07226] [INSPIRE].

[18] F. Caola, G. Luisoni, K. Melnikov and R. Röntsch, NNLO QCD corrections to associated $W H$ production and $H \rightarrow b \bar{b}$ decay, Phys. Rev. D 97 (2018) 074022 [arXiv:1712.06954] [INSPIRE].

[19] R. Gauld, A. Gehrmann-De Ridder, E.W.N. Glover, A. Huss and I. Majer, Associated production of a Higgs boson decaying into bottom quarks and a weak vector boson decaying leptonically at NNLO in QCD, JHEP 10 (2019) 002 [arXiv:1907.05836] [INSPIRE].

[20] R. Mondini, M. Schiavi and C. Williams, $N^{3} L O$ predictions for the decay of the Higgs boson to bottom quarks, JHEP 06 (2019) 079 [arXiv: 1904.08960] [INSPIRE].

[21] W. Bernreuther, L. Chen and Z.-G. Si, Differential decay rates of CP-even and CP-odd Higgs bosons to top and bottom quarks at NNLO QCD, JHEP 07 (2018) 159 [arXiv: 1805. 06658] [INSPIRE].

[22] G. Ferrera, G. Somogyi and F. Tramontano, Associated production of a Higgs boson decaying into bottom quarks at the LHC in full NNLO QCD, Phys. Lett. B 780 (2018) 346 [arXiv: 1705.10304] [INSPIRE]. 
[23] W. Astill, W. Bizoń, E. Re and G. Zanderighi, NNLOPS accurate associated HZ production with $H \rightarrow b \bar{b}$ decay at $N L O$, JHEP 11 (2018) 157 [arXiv: 1804.08141] [INSPIRE].

[24] S. Alioli, A. Broggio, S. Kallweit, M.A. Lim and L. Rottoli, Higgsstrahlung at NNLL'+NNLO matched to parton showers in GENEVA, Phys. Rev. D 100 (2019) 096016 [arXiv: 1909.02026] [INSPIRE].

[25] F. Granata, J.M. Lindert, C. Oleari and S. Pozzorini, $N L O Q C D+E W$ predictions for $H V$ and $H V+$ jet production including parton-shower effects, JHEP 09 (2017) 012 [arXiv: 1706.03522] [INSPIRE].

[26] F. Caola, K. Melnikov and R. Röntsch, Nested soft-collinear subtractions in NNLO QCD computations, Eur. Phys. J. C 77 (2017) 248 [arXiv:1702.01352] [INSPIRE].

[27] F. Caola, K. Melnikov and R. Röntsch, Analytic results for color-singlet production at NNLO QCD with the nested soft-collinear subtraction scheme, Eur. Phys. J. C 79 (2019) 386 [arXiv: 1902.02081] [INSPIRE].

[28] F. Caola, K. Melnikov and R. Röntsch, Analytic results for decays of color singlets to gg and $q \bar{q}$ final states at NNLO QCD with the nested soft-collinear subtraction scheme, Eur. Phys. J. C 79 (2019) 1013 [arXiv: 1907.05398] [InSPIRE].

[29] M. Czakon, A novel subtraction scheme for double-real radiation at NNLO, Phys. Lett. B 693 (2010) 259 [arXiv: 1005. 0274] [inSPIRE].

[30] M. Czakon, Double-real radiation in hadronic top quark pair production as a proof of a certain concept, Nucl. Phys. B 849 (2011) 250 [arXiv:1101.0642] [INSPIRE].

[31] M. Czakon and D. Heymes, Four-dimensional formulation of the sector-improved residue subtraction scheme, Nucl. Phys. B 890 (2014) 152 [arXiv:1408.2500] [INSPIRE].

[32] M. Czakon, A. van Hameren, A. Mitov and R. Poncelet, Single-jet inclusive rates with exact color at $O\left(\alpha_{s}^{4}\right)$, JHEP 10 (2019) 262 [arXiv:1907.12911] [INSPIRE].

[33] G. 't Hooft and M.J.G. Veltman, Regularization and renormalization of gauge fields, Nucl. Phys. B 44 (1972) 189 [inSPIRE].

[34] J.F. Ashmore, A method of gauge invariant regularization, Lett. Nuovo Cim. 4 (1972) 289 [INSPIRE].

[35] G.M. Cicuta and E. Montaldi, Analytic renormalization via continuous space dimension, Lett. Nuovo Cim. 4 (1972) 329 [INSPIRE].

[36] C.G. Bollini and J.J. Giambiagi, Dimensional renormalization: the number of dimensions as a regularizing parameter, Nuovo Cim. B 12 (1972) 20 [INSPIRE].

[37] W.J. Marciano and A. Sirlin, Dimensional regularization of infrared divergences, Nucl. Phys. B 88 (1975) 86 [inSPIRE].

[38] T. Kinoshita, Mass singularities of Feynman amplitudes, J. Math. Phys. 3 (1962) 650 [INSPIRE].

[39] T.D. Lee and M. Nauenberg, Degenerate systems and mass singularities, Phys. Rev. 133 (1964) B1549 [INSPIRE].

[40] T. Binoth and G. Heinrich, An automatized algorithm to compute infrared divergent multiloop integrals, Nucl. Phys. B 585 (2000) 741 [hep-ph/0004013] [INSPIRE].

[41] C. Anastasiou, K. Melnikov and F. Petriello, A new method for real radiation at NNLO, Phys. Rev. D 69 (2004) 076010 [hep-ph/0311311] [INSPIRE]. 
[42] T. Binoth and G. Heinrich, Numerical evaluation of phase space integrals by sector decomposition, Nucl. Phys. B 693 (2004) 134 [hep-ph/0402265] [INSPIRE].

[43] S. Catani, The singular behavior of QCD amplitudes at two loop order, Phys. Lett. B 427 (1998) 161 [hep-ph/9802439] [INSPIRE].

[44] S. Catani, S. Dittmaier and Z. Trócsányi, One loop singular behavior of QCD and SUSY QCD amplitudes with massive partons, Phys. Lett. B 500 (2001) 149 [hep-ph/0011222] [INSPIRE].

[45] S.M. Aybat, L.J. Dixon and G.F. Sterman, The two-loop soft anomalous dimension matrix and resummation at next-to-next-to leading pole, Phys. Rev. D 74 (2006) 074004 [hep-ph/0607309] [INSPIRE].

[46] T. Becher and M. Neubert, Infrared singularities of QCD amplitudes with massive partons, Phys. Rev. D 79 (2009) 125004 [Erratum ibid. D 80 (2009) 109901] [arXiv:0904.1021] [INSPIRE].

[47] M. Czakon, A. Mitov and G.F. Sterman, Threshold resummation for top-pair hadroproduction to next-to-next-to-leading log, Phys. Rev. D 80 (2009) 074017 [arXiv:0907.1790] [INSPIRE].

[48] A. Mitov, G.F. Sterman and I. Sung, The massive soft anomalous dimension matrix at two loops, Phys. Rev. D 79 (2009) 094015 [arXiv: 0903.3241] [INSPIRE].

[49] A. Ferroglia, M. Neubert, B.D. Pecjak and L.L. Yang, Two-loop divergences of massive scattering amplitudes in non-Abelian gauge theories, JHEP 11 (2009) 062 [arXiv:0908.3676] [INSPIRE].

[50] A. Mitov, G.F. Sterman and I. Sung, Computation of the soft anomalous dimension matrix in coordinate space, Phys. Rev. D 82 (2010) 034020 [arXiv: 1005.4646] [INSPIRE].

[51] T. Becher and M. Neubert, Infrared singularities of scattering amplitudes in perturbative QCD, Phys. Rev. Lett. 102 (2009) 162001 [Erratum ibid. 111 (2013) 199905] [arXiv: 0901.0722] [INSPIRE].

[52] S. Catani and M.H. Seymour, A general algorithm for calculating jet cross-sections in NLO QCD, Nucl. Phys. B 485 (1997) 291 [Erratum ibid. B 510 (1998) 503] [hep-ph/9605323] [INSPIRE].

[53] D.J. Broadhurst, N. Gray and K. Schilcher, Gauge invariant on-shell $Z_{2}$ in $Q E D, Q C D$ and the effective field theory of a static quark, Z. Phys. C 52 (1991) 111 [INSPIRE].

[54] N. Gray, D.J. Broadhurst, W. Grafe and K. Schilcher, Three loop relation of quark (modified) $\overline{M S}$ and pole masses, Z. Phys. C 48 (1990) 673 [INSPIRE].

[55] S. Frixione, Z. Kunszt and A. Signer, Three jet cross-sections to next-to-leading order, Nucl. Phys. B 467 (1996) 399 [hep-ph/9512328] [INSPIRE].

[56] S. Frixione, A general approach to jet cross-sections in QCD, Nucl. Phys. B 507 (1997) 295 [hep-ph/9706545] [INSPIRE].

[57] S. Catani and M.H. Seymour, The dipole formalism for the calculation of QCD jet cross-sections at next-to-leading order, Phys. Lett. B 378 (1996) 287 [hep-ph/9602277] [INSPIRE]. 
[58] S. Catani, S. Dittmaier, M.H. Seymour and Z. Trócsányi, The dipole formalism for next-to-leading order QCD calculations with massive partons, Nucl. Phys. B 627 (2002) 189 [hep-ph/0201036] [INSPIRE].

[59] Particle Data Group collaboration, Review of particle physics, Phys. Rev. D 98 (2018) 030001 [INSPIRE].

[60] L.J. Dixon, Calculating scattering amplitudes efficiently, in $Q C D$ and beyond. Proceedings, Theoretical Advanced Study Institute in Elementary Particle Physics, TASI-95, Boulder, CO, U.S.A., 4-30 June 1995, pg. 539 [hep-ph/9601359] [INSPIRE].

[61] M. Brucherseifer, F. Caola and K. Melnikov, $O\left(\alpha_{s}^{2}\right)$ corrections to fully-differential top quark decays, JHEP 04 (2013) 059 [arXiv:1301.7133] [INSPIRE].

[62] G. Passarino and M.J.G. Veltman, One loop corrections for $e^{+} e^{-}$annihilation into $\mu^{+} \mu^{-}$ in the Weinberg model, Nucl. Phys. B 160 (1979) 151 [INSPIRE].

[63] R.K. Ellis and G. Zanderighi, Scalar one-loop integrals for QCD, JHEP 02 (2008) 002 [arXiv:0712.1851] [INSPIRE].

[64] S. Carrazza, R.K. Ellis and G. Zanderighi, QCDLoop: a comprehensive framework for one-loop scalar integrals, Comput. Phys. Commun. 209 (2016) 134 [arXiv:1605.03181] [INSPIRE].

[65] J. Ablinger et al., Heavy quark form factors at two loops, Phys. Rev. D 97 (2018) 094022 [arXiv: 1712.09889] [INSPIRE].

[66] W. Bernreuther, R. Bonciani, T. Gehrmann, R. Heinesch, P. Mastrolia and E. Remiddi, Decays of scalar and pseudoscalar Higgs bosons into fermions: two-loop QCD corrections to the Higgs-quark-antiquark amplitude, Phys. Rev. D 72 (2005) 096002 [hep-ph/0508254] [INSPIRE].

[67] E. Remiddi and J.A.M. Vermaseren, Harmonic polylogarithms, Int. J. Mod. Phys. A 15 (2000) 725 [hep-ph/9905237] [INSPIRE].

[68] T. Gehrmann and E. Remiddi, Numerical evaluation of harmonic polylogarithms, Comput. Phys. Commun. 141 (2001) 296 [hep-ph/0107173] [INSPIRE].

[69] J. Gluza, A. Mitov, S. Moch and T. Riemann, The QCD form factor of heavy quarks at NNLO, JHEP 07 (2009) 001 [arXiv: 0905.1137] [INSPIRE].

[70] K.G. Chetyrkin, J.H. Kühn and M. Steinhauser, RunDec: a Mathematica package for running and decoupling of the strong coupling and quark masses, Comput. Phys. Commun. 133 (2000) 43 [hep-ph/0004189] [INSPIRE].

[71] F. Herren and M. Steinhauser, Version 3 of RunDec and CRunDec, Comput. Phys. Commun. 224 (2018) 333 [arXiv: 1703.03751] [INSPIRE].

[72] K.G. Chetyrkin, Correlator of the quark scalar currents and $\Gamma_{\text {tot }}(H \rightarrow$ hadrons $)$ at $O\left(\alpha_{s}^{3}\right)$ in pQCD, Phys. Lett. B 390 (1997) 309 [hep-ph/9608318] [INSPIRE].

[73] S. Catani, Y.L. Dokshitzer, M. Olsson, G. Turnock and B.R. Webber, New clustering algorithm for multi-jet cross-sections in $e^{+} e^{-}$annihilation, Phys. Lett. B 269 (1991) 432 [INSPIRE].

[74] M. Cacciari, G.P. Salam and G. Soyez, FastJet user manual, Eur. Phys. J. C 72 (2012) 1896 [arXiv:1111.6097] [INSPIRE]. 
[75] D.J. Gross and F. Wilczek, Ultraviolet behavior of non-Abelian gauge theories, Phys. Rev. Lett. 30 (1973) 1343 [INSPIRE].

[76] H.D. Politzer, Reliable perturbative results for strong interactions?, Phys. Rev. Lett. 30 (1973) 1346 [INSPIRE].

[77] K. Melnikov and T. van Ritbergen, The three loop on-shell renormalization of $Q C D$ and QED, Nucl. Phys. B 591 (2000) 515 [hep-ph/0005131] [InSPIRE].

[78] W. Beenakker, S. Dittmaier, M. Krämer, B. Plumper, M. Spira and P.M. Zerwas, NLO

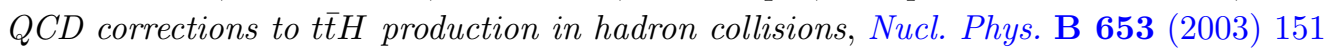
[hep-ph/0211352] [INSPIRE].

[79] M. Czakon, A. Mitov and S. Moch, Heavy-quark production in gluon fusion at two loops in QCD, Nucl. Phys. B 798 (2008) 210 [arXiv:0707.4139] [inSPIRE].

[80] S. Weinberg, Effective gauge theories, Phys. Lett. B 91 (1980) 51 [INSPIRE].

[81] B.A. Ovrut and H.J. Schnitzer, The decoupling theorem and minimal subtraction, Phys. Lett. B 100 (1981) 403 [INSPIRE].

[82] W. Wetzel, Minimal subtraction and the decoupling of heavy quarks for arbitrary values of the gauge parameter, Nucl. Phys. B 196 (1982) 259 [INSPIRE].

[83] W. Bernreuther and W. Wetzel, Decoupling of heavy quarks in the minimal subtraction scheme, Nucl. Phys. B 197 (1982) 228 [Erratum ibid. B 513 (1998) 758] [inSPIRE].

[84] W. Bernreuther, Decoupling of heavy quarks in quantum chromodynamics, Annals Phys. 151 (1983) 127 [INSPIRE].

[85] W. Bernreuther, Heavy quark effects on the parameters of quantum chromodynamics defined by minimal subtraction, Z. Phys. C 20 (1983) 331 [InSPIRE].

[86] K.G. Chetyrkin, B.A. Kniehl and M. Steinhauser, Decoupling relations to $O\left(\alpha_{s}^{3}\right)$ and their connection to low-energy theorems, Nucl. Phys. B 510 (1998) 61 [hep-ph/9708255] [INSPIRE].

[87] M. Gerlach, G. Mishima and M. Steinhauser, Matching coefficients in nonrelativistic QCD to two-loop accuracy, Phys. Rev. D 100 (2019) 054016 [arXiv: 1907.08227] [INSPIRE].

[88] K. Melnikov and T.v. Ritbergen, The three loop relation between the MS-bar and the pole quark masses, Phys. Lett. B 482 (2000) 99 [hep-ph/9912391] [INSPIRE].

[89] A. Mitov and S. Moch, The singular behavior of massive QCD amplitudes, JHEP 05 (2007) 001 [hep-ph/0612149] [INSPIRE].

[90] I. Bierenbaum, M. Czakon and A. Mitov, The singular behavior of one-loop massive QCD amplitudes with one external soft gluon, Nucl. Phys. B 856 (2012) 228 [arXiv:1107.4384] [INSPIRE].

[91] M.L. Czakon and A. Mitov, A simplified expression for the one-loop soft-gluon current with massive fermions, arXiv: 1804.02069 [INSPIRE].

[92] S. Catani and M. Grazzini, Infrared factorization of tree level QCD amplitudes at the next-to-next-to-leading order and beyond, Nucl. Phys. B 570 (2000) 287 [hep-ph/9908523] [INSPIRE].

[93] S. Alioli, P. Nason, C. Oleari and E. Re, A general framework for implementing NLO calculations in shower Monte Carlo programs: the POWHEG BOX, JHEP 06 (2010) 043 [arXiv: 1002 .2581] [INSPIRE]. 
[94] J. Ablinger, A computer algebra toolbox for harmonic sums related to particle physics, Diploma thesis, Linz U., Linz, Austria (2009) [arXiv: 1011.1176] [INSPIRE].

[95] J. Ablinger, Computer algebra algorithms for special functions in particle physics, Ph.D. thesis, Linz U., Linz, Austria (2012) [arXiv:1305.0687] [INSPIRE].

[96] J.A.M. Vermaseren, Harmonic sums, Mellin transforms and integrals, Int. J. Mod. Phys. A 14 (1999) 2037 [hep-ph/9806280] [INSPIRE].

[97] J. Blümlein, Structural relations of harmonic sums and Mellin transforms up to weight $w=5$, Comput. Phys. Commun. 180 (2009) 2218 [arXiv:0901.3106] [InSPIRE].

[98] J. Ablinger, J. Blümlein and C. Schneider, Harmonic sums and polylogarithms generated by cyclotomic polynomials, J. Math. Phys. 52 (2011) 102301 [arXiv:1105.6063] [INSPIRE].

[99] J. Ablinger, J. Blümlein and C. Schneider, Analytic and algorithmic aspects of generalized harmonic sums and polylogarithms, J. Math. Phys. 54 (2013) 082301 [arXiv:1302.0378] [INSPIRE].

[100] J. Ablinger, The package HarmonicSums: computer algebra and analytic aspects of nested sums, PoS (LL2014) 019 (2014) [arXiv:1407.6180] [INSPIRE].

[101] J. Ablinger, Inverse Mellin transform of holonomic sequences, PoS(LL2016) 067 (2016) [arXiv: 1606.02845].

[102] J. Ablinger, Computing the inverse Mellin transform of holonomic sequences using Kovacic's algorithm, PoS (RADCOR2017) 069 (2017) [arXiv: 1801.01039].

[103] J. Ablinger, Discovering and proving infinite Pochhammer sum identities, arXiv: 1902.11001 [INSPIRE]. 